\title{
SUPPLEMENTARY MATERIAL FOR: OPTIMAL COMPUTATIONAL AND STATISTICAL RATES OF CONVERGENCE FOR SPARSE NONCONVEX LEARNING PROBLEMS
}

\author{
By ZhaOran Wang*, Han LiU* and Tong Zhang ${ }^{\dagger}$
}

\section{APPENDIX A: NONCONVEX PENALTY AND LOSS FUNCTIONS}

We provide detailed descriptions of the nonconvex penalty and loss functions discussed in $\S 2$ of Wang et al. (2014a). Specifically, for the nonconvex penalties, i.e., SCAD and MCP, we provide their analytical forms in $\S \mathrm{A} .1$, and illustrate regularity condition (e) of Wang et al. (2014a) in §A.2. For the nonconvex loss, i.e., semiparametric elliptical design loss, we provide further details on elliptical distribution in $\S$ A.3, and define the two-step elliptical covariance matrix estimation procedure for semiparametric elliptical design regression in $\S$ A.4.

A.1. Analytical Forms of SCAD and MCP. The SCAD penalty in (2.1) of Wang et al. (2014a) can be written as

$$
\begin{aligned}
p_{\lambda}\left(\beta_{j}\right)=\lambda\left|\beta_{j}\right| \cdot \mathbb{1}\left(\left|\beta_{j}\right| \leq \lambda\right) & -\frac{\beta_{j}^{2}-2 a \lambda\left|\beta_{j}\right|+\lambda^{2}}{2(a-1)} \cdot \mathbb{1}\left(\lambda<\left|\beta_{j}\right| \leq a \lambda\right) \\
+ & \frac{(a+1) \lambda^{2}}{2} \cdot \mathbb{1}\left(\left|\beta_{j}\right|>a \lambda\right), \quad a>2,
\end{aligned}
$$

and the MCP penalty in (2.2) of Wang et al. (2014a) can be written as

$$
p_{\lambda}\left(\beta_{j}\right)=\left(\lambda\left|\beta_{j}\right|-\frac{\beta_{j}^{2}}{2 b}\right) \cdot \mathbb{1}\left(\left|\beta_{j}\right| \leq b \lambda\right)+\frac{b \lambda^{2}}{2} \cdot \mathbb{1}\left(\left|\beta_{j}\right|>b \lambda\right), \quad b>0 .
$$

Correspondingly, the specific forms of the concave component $q_{\lambda}\left(\beta_{j}\right)$ are

$$
q_{\lambda}\left(\beta_{j}\right)=\left\{\begin{array}{rr}
\frac{2 \lambda\left|\beta_{j}\right|-\beta_{j}^{2}-\lambda^{2}}{2(a-1)} \cdot \mathbb{1}\left(\lambda<\left|\beta_{j}\right| \leq a \lambda\right) \\
\quad+\frac{(a+1) \lambda^{2}-2 \lambda\left|\beta_{j}\right|}{2} \cdot \mathbb{1}\left(\left|\beta_{j}\right|>a \lambda\right), & \text { SCAD, } \\
-\frac{\beta_{j}^{2}}{2 b} \cdot \mathbb{1}\left(\left|\beta_{j}\right| \leq b \lambda\right)+\left(\frac{b \lambda^{2}}{2}-\lambda\left|\beta_{j}\right|\right) \cdot \mathbb{1}\left(\left|\beta_{j}\right|>b \lambda\right), & \text { MCP. }
\end{array}\right.
$$

A.2. Illustration of Regularity Condition (e) for SCAD and MCP. We verify regularity condition (e) in Wang et al. (2014a) holds for SCAD 
and MCP in Figure 4: For MCP, we illustrate with Figure 4(a); For SCAD, we illustrate with Figure 4(b) (for $\lambda_{2} \geq a \lambda_{1}$ ) and Figure 4(c).

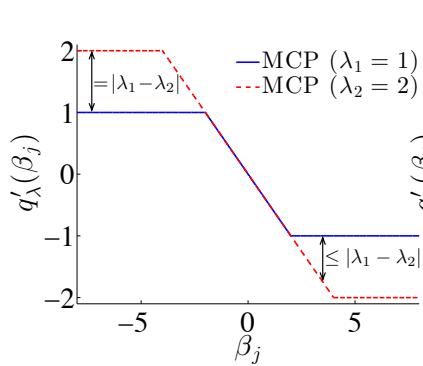

(a)

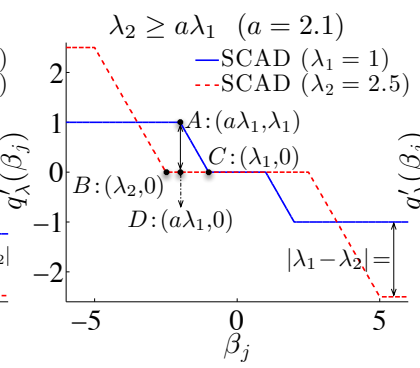

(b)

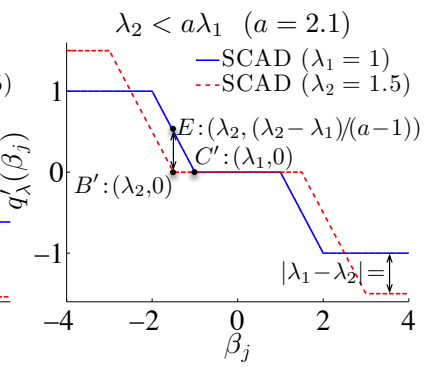

(c)

FIG 4. An illustration of regularity condition (e) in Wang et al. (2014a) for MCP and SCAD: (a) Plots of $q_{\lambda_{1}}^{\prime}\left(\beta_{j}\right)$ and $q_{\lambda_{2}}^{\prime}\left(\beta_{j}\right)$ for $M C P$ with $\lambda_{1}=1, \lambda_{2}=2$ and $b=2$; (b) Plots of $q_{\lambda_{1}}^{\prime}\left(\beta_{j}\right)$ and $q_{\lambda_{2}}^{\prime}\left(\beta_{j}\right)$ for SCAD with $\lambda_{1}=1, \lambda_{2}=2.5$ and $a=2.1$; (c) Plots of $q_{\lambda_{1}}^{\prime}\left(\beta_{j}\right)$ and $q_{\lambda_{2}}^{\prime}\left(\beta_{j}\right)$ for $S C A D$ with $\lambda_{1}=1, \lambda_{2}=1.5$ and $a=2.1$. Subfigure (a) shows that regularity condition (e) holds for MCP. For SCAD, we consider two cases: $\lambda_{2} \geq a \lambda_{1}$, as illustrated in (b); $\lambda_{2}<a \lambda_{1}$ as illustrated in (c). In the first case, $|A D|=\lambda_{1} \leq(a-1) \lambda_{1} \leq\left|\lambda_{1}-\lambda_{2}\right|$ since $a>2$ and $\lambda_{2} \geq a \lambda_{1}$. In the second case, $\left|B^{\prime} E\right|=\left(\lambda_{2}-\lambda_{1}\right) /(a-1) \leq\left|\lambda_{1}-\lambda_{2}\right|$, because the slope of $E C^{\prime}$ is $(-1 /(a-1))$ with $a>2$.

A.3. Elliptical Distribution. Before we present the definition of elliptical distribution, we first introduce some notation: If random vectors $\boldsymbol{Z}_{1}$ and $Z_{2}$ have the same distribution, we denote by $\boldsymbol{Z}_{1} \stackrel{d}{=} \boldsymbol{Z}_{2}$; The $d$-dimensional $\ell_{2}$ unit sphere $\left\{\boldsymbol{v}:\|\boldsymbol{v}\|_{2}=1, \boldsymbol{v} \in \mathbb{R}^{d}\right\}$ is denoted by $\mathbb{S}^{d-1}$; For a matrix $\mathbf{M} \in \mathbb{R}^{d \times d}$, we define $\operatorname{diag}(\mathbf{M})$ to be a diagonal matrix with diagonal entries $[\operatorname{diag}(\mathbf{M})]_{j j}=\mathbf{M}_{j j}(j=1, \ldots, d)$.

Definition A.1 (Elliptical distribution). For $\boldsymbol{\mu}=\left(\mu_{1}, \ldots, \mu_{d}\right)^{T} \in \mathbb{R}^{d}$ and $\boldsymbol{\Sigma} \in \mathbb{R}^{d \times d}$ with $\operatorname{rank}(\boldsymbol{\Sigma})=r \leq d$, a random vector $\boldsymbol{W}=\left(W_{1}, \ldots, W_{d}\right)^{T}$ follows an elliptical distribution, denoted by $\operatorname{EC}_{d}(\boldsymbol{\mu}, \boldsymbol{\Sigma}, \Xi)$, if and only if

$$
\boldsymbol{W} \stackrel{d}{=} \boldsymbol{\mu}+\Xi \mathbf{A} \boldsymbol{U}
$$

Here $\boldsymbol{U}$ is a random vector uniformly distributed on the unit sphere $\mathbb{S}^{r-1} ; \Xi \geq$ 0 is a scalar random variable independent of $\boldsymbol{U} ; \mathbf{A} \in \mathbb{R}^{d \times r}$ is a deterministic matrix such that $\mathbf{A} \mathbf{A}^{T}=\boldsymbol{\Sigma}$. We call $\boldsymbol{\Sigma}$ the scatter matrix. The generalized correlation matrix is defined as $\boldsymbol{\Sigma}^{0}=\operatorname{diag}(\boldsymbol{\Sigma})^{-1 / 2} \cdot \boldsymbol{\Sigma} \cdot \operatorname{diag}(\boldsymbol{\Sigma})^{-1 / 2}$. When $\mathbb{E}\left(\Xi^{2}\right)$ exists, $\boldsymbol{\Sigma}^{0}$ is the correlation matrix of $\boldsymbol{W}$.

Remark A.2. Note that simultaneously scaling $\Xi$ and $\boldsymbol{U}$ (e.g., $\Xi \rightarrow \Xi / C$ and $\boldsymbol{U} \rightarrow \boldsymbol{U} / C$, where $C$ is a constant) leads to the same elliptical distribution. To make this model identifiable, we assume $\mu_{j}=\mathbb{E}\left(W_{j}\right)$ and $\boldsymbol{\Sigma}_{j j}=\operatorname{Var}\left(W_{j}\right)$. 
Remark A.3. The elliptical distribution family includes a variety of possibly heavy-tail distributions: multivariate Gaussian, multivariate Cauchy, Student's t, logistic, Kotz, symmetric Pearson type-II and type-VII distributions.

A.4. Rank-based Covariance Matrix Estimation for Semiparametric Elliptical Design Regression. We provide details of the two-step procedure for estimating the covariance matrix of the elliptically distributed random vector $\boldsymbol{Z}=\left(Y, \boldsymbol{X}^{T}\right)^{T} \in \mathbb{R}^{d+1}$ discussed in $\S 2.2$ of Wang et al. (2014a):

\section{Elliptical Covariance Matrix Estimation}

S1. First, we define a rank-based estimator $\widehat{\mathbf{R}}_{Z}$ of the generalized correlation matrix $\boldsymbol{\Sigma}_{\boldsymbol{Z}}^{0}$ using the Kendall's tau statistic. Let $\mathbf{z}_{1}, \ldots, \mathbf{z}_{n} \in \mathbb{R}^{d+1}$ with $\mathbf{z}_{i}=\left(z_{i 1}, \ldots, z_{i(d+1)}\right)^{T}$ be $n$ independent observations of $\boldsymbol{Z}$. The Kendall's tau correlation coefficient is defined as

$$
\widehat{\tau}_{j k}\left(\mathbf{z}_{1}, \ldots, \mathbf{z}_{n}\right)= \begin{cases}\sum_{1 \leq i<i^{\prime}<n} \frac{2 \operatorname{sign}\left(z_{i j}-z_{i^{\prime} j}\right) \operatorname{sign}\left(z_{i k}-z_{i^{\prime} k}\right)}{n(n-1)}, & \text { for } j \neq k, \\ 1, & \text { for } j=k .\end{cases}
$$

We define the Kendall's tau correlation matrix estimator as

$$
\widehat{\mathbf{R}}_{\boldsymbol{Z}}=\left[\left(\widehat{\mathbf{R}}_{\boldsymbol{Z}}\right)_{j k}\right]=\left[\sin \left(\frac{\pi}{2} \widehat{\tau}_{j k}\left(\mathbf{z}_{1}, \ldots, \mathbf{z}_{n}\right)\right)\right] \text {. }
$$

Liu et al. (2012); Han and Liu $(2012,2013)$ showed that $\widehat{\mathbf{R}}_{\boldsymbol{Z}}$ is a robust estimator of the population generalized correlation matrix $\boldsymbol{\Sigma}_{\boldsymbol{Z}}^{0}$, and is invariant to different distributions of the generating variable $\Xi$ within the whole elliptical family.

S2. Second, we construct a covariance matrix estimator

$$
\widehat{\mathbf{K}}_{\boldsymbol{Z}}=\left[\left(\widehat{\mathbf{K}}_{\boldsymbol{Z}}\right)_{j k}\right]=\left[\left(\widehat{\mathbf{R}}_{\boldsymbol{Z}}\right)_{j k} \cdot \widehat{\sigma}_{j} \widehat{\sigma}_{k}\right],
$$

where $\widehat{\sigma}_{1}, \ldots, \widehat{\sigma}_{d+1}$ are the estimators of the standard deviations of $Z_{1}, \ldots, Z_{d+1}$. We calculate $\widehat{\sigma}_{1}, \ldots, \widehat{\sigma}_{d+1}$ using the Catoni's $M$-estimator (Catoni, 2012) described in $\S \mathrm{E}$. The main advantage of the Cantoni's estimator is that, for a fixed level of confidence, it achieves the same deviation behavior as a Gaussian random variable under a weak moment condition. 


\section{APPENDIX B: PROXIMAL-GRADIENT METHOD FOR NONCONVEX PROBLEMS}

We provide details of the proximal-gradient method tailored to nonconvex problems. In particular, in $\S \mathrm{B} .1$ we provide the optimization update schemes for the specific nonconvex problems discussed in $\S 2$ of Wang et al. (2014a). In $\S$ B. 2 we provide the detailed derivation of the closed-form expression of update scheme (3.9) in Wang et al. (2014a).

B.1. Optimization Update Schemes for Specific Nonconvex Problems. To obtain the specific optimization update schemes of the proximalgradient method for the nonconvex problems discussed in $\S 2$ of Wang et al. (2014a), we only need to plug the following specific definitions of $\nabla \mathcal{L}(\boldsymbol{\beta})$ and $\nabla \mathcal{Q}_{\lambda_{t}}(\boldsymbol{\beta})$ into (3.11) of Wang et al. (2014a):

- For the (nonconvex) loss functions discussed in $\S 2$ of Wang et al. (2014a),

$$
\nabla \mathcal{L}(\boldsymbol{\beta})=\left\{\begin{array}{lr}
\frac{1}{n} \mathbf{X}^{T}(\mathbf{X} \boldsymbol{\beta}-\mathbf{y}), & \text { least squares loss } \\
\frac{1}{n} \sum_{i=1}^{n} \mathbf{x}_{i}\left(\frac{\exp \left(\mathbf{x}_{i}^{T} \boldsymbol{\beta}\right)}{1+\exp \left(\mathbf{x}_{i}^{T} \boldsymbol{\beta}\right)}-y_{i}\right), & \text { logistic loss } \\
\widehat{\mathbf{K}}_{\boldsymbol{X}} \boldsymbol{\beta}-\widehat{\mathbf{K}}_{\boldsymbol{X}, Y}, & \text { semiparametric elliptical design loss }
\end{array}\right.
$$

where $\widehat{\mathbf{K}}_{\boldsymbol{X}} \in \mathbb{R}^{d \times d}$ and $\widehat{\mathbf{K}}_{\boldsymbol{X}, Y} \in \mathbb{R}^{d \times 1}$ are the submatrices of $\widehat{\mathbf{K}}_{\boldsymbol{Z}}$, which is the semiparametric elliptical covariance matrix estimator defined in (A.2). More specifically,

$$
\widehat{\mathbf{K}}_{\boldsymbol{Z}}=\left(\begin{array}{cc}
\widehat{\mathbf{K}}_{Y} & \widehat{\mathbf{K}}_{\boldsymbol{X}, Y}^{T} \\
\widehat{\mathbf{K}}_{\boldsymbol{X}, Y} & \widehat{\mathbf{K}}_{\boldsymbol{X}}
\end{array}\right) .
$$

- For the nonconvex penalty functions discussed in $\S 2$ of Wang et al. (2014a),

$$
\left(\nabla \mathcal{Q}_{\lambda_{t}}(\boldsymbol{\beta})\right)_{j}=\left\{\begin{array}{c}
\frac{\lambda_{t} \operatorname{sign}\left(\beta_{j}\right)-\beta_{j}}{a-1} \cdot \mathbb{1}\left(\lambda_{t}<\left|\beta_{j}\right| \leq a \lambda_{t}\right) \\
-\lambda_{t} \operatorname{sign}\left(\beta_{j}\right) \cdot \mathbb{1}\left(\left|\beta_{j}\right|>a \lambda_{t}\right), \quad \text { SCAD, } \\
-\frac{\beta_{j}}{b} \lambda_{t} \operatorname{sign}\left(\beta_{j}\right) \cdot \mathbb{1}\left(\left|\beta_{j}\right| \leq b \lambda_{t}\right) \\
-\lambda_{t} \operatorname{sign}\left(\beta_{j}\right) \cdot \mathbb{1}\left(\left|\beta_{j}\right|>b \lambda_{t}\right), \quad \mathrm{MCP},
\end{array}\right.
$$

where $a>2, b>0$.

B.2. Derivation of Optimization Update Schemes. For notational simplicity, we denote $L_{t}^{k}$ by $L, \boldsymbol{\beta}_{t}^{k-1}$ by $\boldsymbol{\beta}^{\prime}$, and $\lambda_{t}$ by $\lambda$ in the rest of this 
section.

Derivation of (3.10) of Wang et al. (2014a): If $\Omega=\mathbb{R}^{d}$, then we have $\mathcal{T}_{L, \lambda}\left(\boldsymbol{\beta}^{\prime} ;+\infty\right)=\underset{\boldsymbol{\beta} \in \mathbb{R}^{d}}{\operatorname{argmin}}\left\{\psi_{L, \lambda}\left(\boldsymbol{\beta} ; \boldsymbol{\beta}^{\prime}\right)\right\}$

$$
\begin{aligned}
& =\underset{\boldsymbol{\beta} \in \mathbb{R}^{d}}{\operatorname{argmin}}\left\{\widetilde{\mathcal{L}}_{\lambda}\left(\boldsymbol{\beta}^{\prime}\right)+\nabla \widetilde{\mathcal{L}}_{\lambda}\left(\boldsymbol{\beta}^{\prime}\right)^{T}\left(\boldsymbol{\beta}-\boldsymbol{\beta}^{\prime}\right)+\frac{L}{2}\left\|\boldsymbol{\beta}-\boldsymbol{\beta}^{\prime}\right\|_{2}^{2}+\lambda\|\boldsymbol{\beta}\|_{1}\right\} \\
& =\underset{\boldsymbol{\beta} \in \mathbb{R}^{d}}{\operatorname{argmin}}\left\{\frac{1}{2}\|\boldsymbol{\beta}-\underbrace{\left(\boldsymbol{\beta}^{\prime}-\frac{1}{L} \nabla \widetilde{\mathcal{L}_{\lambda}}\left(\boldsymbol{\beta}^{\prime}\right)\right)}_{\overline{\boldsymbol{\beta}}}\|_{2}^{2}+\frac{\lambda}{L}\|\boldsymbol{\beta}\|_{1}\right\} .
\end{aligned}
$$

It is known that the minimizer of (B.2) can be obtained by soft-thresholding $\overline{\boldsymbol{\beta}}$ with the threshold of value $\lambda / L$, i.e.,

$$
\left(\mathcal{T}_{L, \lambda}\left(\boldsymbol{\beta}^{\prime} ;+\infty\right)\right)_{j}= \begin{cases}0 & \text { if }\left|\bar{\beta}_{j}\right| \leq \lambda / L \\ \operatorname{sign}\left(\bar{\beta}_{j}\right)\left(\left|\bar{\beta}_{j}\right|-\lambda / L\right) & \text { if }\left|\bar{\beta}_{j}\right|>\lambda / L\end{cases}
$$

Therefore we obtain the first update scheme (3.10) in Wang et al. (2014a) for $\Omega=\mathbb{R}^{d}$.

Derivation of (3.12) of Wang et al. (2014a): If $\Omega=B_{2}(R)=\left\{\boldsymbol{\beta}:\|\boldsymbol{\beta}\|_{2}^{2} \leq R^{2}\right\}$, by Lagrangian duality we can transform the original optimization problem with constraint into an unconstraint optimization problem. Hence, there exists a Lagrangian multiplier $\tau \geq 0$ such that

$$
\mathcal{T}_{L, \lambda}\left(\boldsymbol{\beta}^{\prime} ; R\right)=\underset{\boldsymbol{\beta} \in B_{2}(R)}{\operatorname{argmin}}\left\{\psi_{L, \lambda}\left(\boldsymbol{\beta} ; \boldsymbol{\beta}^{\prime}\right)\right\}=\underset{\boldsymbol{\beta} \in \mathbb{R}^{d}}{\operatorname{argmin}}\left\{\psi_{L, \lambda}\left(\boldsymbol{\beta} ; \boldsymbol{\beta}^{\prime}\right)+\frac{\tau}{2}\|\boldsymbol{\beta}\|_{2}^{2}\right\} .
$$

Consequently, based on (B.2) we have

$$
\begin{aligned}
& \mathcal{T}_{L, \lambda}\left(\boldsymbol{\beta}^{\prime} ; R\right)= \underset{\boldsymbol{\beta} \in \mathbb{R}^{d}}{\operatorname{argmin}}\left\{\widetilde{\mathcal{L}}_{\lambda}\left(\boldsymbol{\beta}^{\prime}\right)+\nabla \widetilde{\mathcal{L}_{\lambda}}\left(\boldsymbol{\beta}^{\prime}\right)^{T}\left(\boldsymbol{\beta}-\boldsymbol{\beta}^{\prime}\right)\right. \\
&\left.+\frac{L}{2}\left\|\boldsymbol{\beta}-\boldsymbol{\beta}^{\prime}\right\|_{2}^{2}+\lambda\|\boldsymbol{\beta}\|_{1}+\frac{\tau}{2}\|\boldsymbol{\beta}\|_{2}^{2}\right\} \\
&= \underset{\boldsymbol{\beta} \in \mathbb{R}^{d}}{\operatorname{argmin}}\left\{\frac{L+\tau}{2}\|\boldsymbol{\beta}\|_{2}^{2}-\left(L \cdot \boldsymbol{\beta}^{\prime}-\nabla \widetilde{\mathcal{L}}_{\lambda}\left(\boldsymbol{\beta}^{\prime}\right)\right)^{T} \boldsymbol{\beta}+\lambda\|\boldsymbol{\beta}\|_{1}\right\} \\
&(\text { B.4 }) \quad \underset{\boldsymbol{\beta} \in \mathbb{R}^{d}}{\operatorname{argmin}}\left\{\frac{1}{2}\|\boldsymbol{\beta}-\underbrace{\left(\frac{L}{L+\tau} \boldsymbol{\beta}^{\prime}-\frac{1}{L+\tau} \nabla \widetilde{\mathcal{L}}_{\lambda}\left(\boldsymbol{\beta}^{\prime}\right)\right)}_{\frac{L}{L+\tau} \overline{\boldsymbol{\beta}}}\|_{2}^{2}+\frac{\lambda}{L+\tau}\|\boldsymbol{\beta}\|_{1}\right\},
\end{aligned}
$$

where $\overline{\boldsymbol{\beta}}=\boldsymbol{\beta}^{\prime}-\nabla \widetilde{\mathcal{L}}_{\lambda}\left(\boldsymbol{\beta}^{\prime}\right) / L$. The minimizer of (B.4) can also be obtained by 
soft-thresholding, i.e.,

$$
\begin{aligned}
& \left(\mathcal{T}_{L, \lambda}\left(\boldsymbol{\beta}^{\prime} ; R\right)\right)_{j} \\
& = \begin{cases}0 & \text { if } \frac{L}{L+\tau}\left|\bar{\beta}_{j}\right| \leq \frac{\lambda}{L+\tau}, \\
\operatorname{sign}\left(\frac{L}{L+\tau} \bar{\beta}_{j}\right)\left(\frac{L}{L+\tau}\left|\bar{\beta}_{j}\right|-\frac{\lambda}{L+\tau}\right) & \text { if } \frac{L}{L+\tau}\left|\bar{\beta}_{j}\right|>\frac{\lambda}{L+\tau} .\end{cases}
\end{aligned}
$$

Comparing (B.5) with (B.3), we have

$$
\mathcal{T}_{L, \lambda}\left(\boldsymbol{\beta}^{\prime} ; R\right)=\frac{L}{L+\tau} \mathcal{T}_{L, \lambda}\left(\boldsymbol{\beta}^{\prime} ;+\infty\right)
$$

Thus, we can obtain the constraint solution $\mathcal{T}_{L, \lambda}\left(\boldsymbol{\beta}^{\prime} ; R\right)$ by first calculating the unconstraint solution $\mathcal{T}_{L, \lambda}\left(\boldsymbol{\beta}^{\prime} ;+\infty\right)$, and then rescaling it by a factor of $L /(L+\tau)$. Note that here the Lagrangian multiplier $\tau$ is unknown. We discuss the following two cases:

- If the constraint $\boldsymbol{\beta} \in B_{2}(R)$ is inactive, then we have $\tau=0$ by complementary slackness, which implies $\mathcal{T}_{L, \lambda}\left(\boldsymbol{\beta}^{\prime} ; R\right)=\mathcal{T}_{L, \lambda}\left(\boldsymbol{\beta}^{\prime} ;+\infty\right)$. Since the constraint is inactive, we have $\left\|\mathcal{T}_{L, \lambda}\left(\boldsymbol{\beta}^{\prime} ; R\right)\right\|_{2}=\left\|\mathcal{T}_{L, \lambda}\left(\boldsymbol{\beta}^{\prime} ;+\infty\right)\right\|_{2}<R$.

- If the constraint $\boldsymbol{\beta} \in B_{2}(R)$ is active, then we have $\tau \geq 0$ by complementary slackness. In this case, the minimizer $\mathcal{T}_{L, \lambda}\left(\boldsymbol{\beta}^{\prime} ; R\right)$ lies on the boundary of $B_{2}(R)$. By (B.6) we have

$$
\left\|\mathcal{T}_{L, \lambda}\left(\boldsymbol{\beta}^{\prime} ;+\infty\right)\right\|_{2}=\frac{L+\tau}{L}\left\|\mathcal{T}_{L, \lambda}\left(\boldsymbol{\beta}^{\prime} ; R\right)\right\|_{2}=\frac{L+\tau}{L} R \geq R
$$

To obtain $\mathcal{T}_{L, \lambda}\left(\boldsymbol{\beta}^{\prime} ; R\right)$, we project $\mathcal{T}_{L, \lambda}\left(\boldsymbol{\beta}^{\prime} ;+\infty\right)$ onto $B_{2}(R)$, which can be achieved by setting $\mathcal{T}_{L, \lambda}\left(\boldsymbol{\beta}^{\prime} ; R\right)=R \cdot \mathcal{T}_{L, \lambda}\left(\boldsymbol{\beta}^{\prime} ;+\infty\right) /\left\|\mathcal{T}_{L, \lambda}\left(\boldsymbol{\beta}^{\prime} ;+\infty\right)\right\|_{2}$.

Therefore we obtain the second update scheme (3.12) in Wang et al. (2014a) for $\Omega=B_{2}(R)$.

\section{APPENDIX C: JUSTIFICATION OF ASSUMPTIONS}

In $\S$ C. 1 and $\S$ C.2, we prove that Assumption 4.1 and Assumption 4.4 in Wang et al. (2014a) hold with high probability respectively.

C.1. Justification of Assumption 4.1 in Wang et al. (2014a). Recall that Assumption 4.1 states that $\left\|\nabla \mathcal{L}\left(\boldsymbol{\beta}^{*}\right)\right\|_{\infty}$ can be upper bounded by $\lambda_{\text {tgt }} / 8$. First we provide two lemmas on the upper bound of $\left\|\nabla \mathcal{L}\left(\boldsymbol{\beta}^{*}\right)\right\|_{\infty}$.

Lemma C.1. For least squares regression with sub-Gaussian noise and logistic regression, we assume that the columns of $\mathbf{X}$ are normalized in such 
a way that $\max _{j \in\{1, \ldots, d\}}\left\{\left\|\mathbf{X}_{j}\right\|_{2}\right\} \leq \sqrt{n}$. Then we have

$$
\left\|\nabla \mathcal{L}\left(\boldsymbol{\beta}^{*}\right)\right\|_{\infty} \leq C \sqrt{\frac{\log d}{n}}
$$

with probability at least $1-d^{-1}$, where $C$ is a constant.

Proof. See Candés and Tao (2007); Zhang and Huang (2008); Zhang (2009); Bickel et al. (2009); Koltchinskii (2009a); Negahban et al. (2012); Wainwright (2009) for a detailed proof.

Lemma C.2. For semiparametric elliptical design regression, we have

$$
\left\|\nabla \mathcal{L}\left(\boldsymbol{\beta}^{*}\right)\right\|_{\infty} \leq C\left\|\boldsymbol{\beta}^{*}\right\|_{1} \sqrt{\frac{\log d}{n}}
$$

with probability at least $1-(d+1)^{-5 / 2}-2(d+1)^{-3}$, where $C$ is a constant.

PROOF. See $\S$ E.3 for a detailed proof.

Recall in Assumption 4.1 of Wang et al. (2014a) we set $\lambda_{\operatorname{tgt}}=C \sqrt{\log d / n}$ for least squares and logistic loss, and $\lambda_{\operatorname{tgt}}=C^{\prime}\left\|\boldsymbol{\beta}^{*}\right\|_{1} \sqrt{\log d / n}$ for semiparametric elliptical design loss. Thus, according to Lemma C.1 and Lemma C.2, $\left\|\nabla \mathcal{L}\left(\boldsymbol{\beta}^{*}\right)\right\|_{\infty} \leq \lambda_{\text {tgt }} / 8$ holds with high probability, i.e., Assumption 4.1 holds with high probability.

C.2. Justification of Assumption 4.4 in Wang et al. (2014a). In this section we show that, for semiparametric elliptical design loss and logistic loss, Assumption 4.4 holds with high probability.

Semiparametric Elliptical Design Loss: First we provide the following lemma on the largest and smallest sparse eigenvalues of the Hessian matrix $\nabla^{2} \mathcal{L}(\boldsymbol{\beta})$ of semiparametric elliptical design loss.

Let $n$ be the sample size, $d$ be the dimension of $\boldsymbol{\beta}$, and $\boldsymbol{Z} \in \mathbb{R}^{d+1}$ be the elliptically distributed random vector in $\S 2.2$ of Wang et al. (2014a). The corresponding covariance matrix estimator $\widehat{\mathbf{K}}_{\boldsymbol{Z}} \in \mathbb{R}^{(d+1) \times(d+1)}$ is defined in (A.2), while its submatrix $\widehat{\mathbf{K}}_{\boldsymbol{X}} \in \mathbb{R}^{d \times d}$ is defined in (B.1). Hence, the Hessian matrix of semiparametric elliptical design loss is $\nabla^{2} \mathcal{L}(\boldsymbol{\beta})=\widehat{\mathbf{K}}_{\boldsymbol{X}}$. Let $s$ the sparsity level.

Lemma C.3. Under suitable conditions (see Han and Liu (2013) for details), for a sufficiently large $n$, there exists an $s$ such that $\rho_{-}\left(\nabla^{2} \mathcal{L}, s\right)>0$ and $\rho_{+}\left(\nabla^{2} \mathcal{L}, s\right)<+\infty$ with probability at least $1-4 d^{-1}-6 d^{-2}$. Here $\rho_{+}\left(\nabla^{2} \mathcal{L}, s\right)$ and $\rho_{-}\left(\nabla^{2} \mathcal{L}, s\right)$ are defined in Definition 4.2 of Wang et al. (2014a). 
PROOF. See $\S$ E.2 for a detailed proof.

Equipped with Lemma C.3, we can justify Assumption 4.4. Recall $s^{*}=$ $\left\|\boldsymbol{\beta}^{*}\right\|_{0}$, where $\boldsymbol{\beta}^{*}$ is the true parameter vector. Suppose that Lemma C.3 holds with $s=C s^{*}, \rho_{+}\left(\nabla^{2} \mathcal{L}, s\right)=C^{\prime}$ and $\rho_{-}\left(\nabla^{2} \mathcal{L}, s\right)=C^{\prime \prime}$, where $C$ satisfies

$$
C \geq 2\left(144 \cdot\left(\frac{2 C^{\prime}}{C^{\prime \prime}}\right)^{2}+250 \cdot\left(\frac{2 C^{\prime}}{C^{\prime \prime}}\right)\right)+1
$$

Meanwhile, let the concavity parameters of the nonconvex penalty be $\zeta_{+}=0$ and $\zeta_{-}=C^{\prime \prime} / 2$. In the sequel we verify that, there exists an integer $\widetilde{s}=$ $(C-1) / 2 \cdot s^{*}$, where $C$ satisfies (C.3), that satisfies Assumption 4.4. Note that the condition number $\kappa$ defined in (4.5) is

$$
\begin{aligned}
\kappa=\frac{\rho_{+}\left(\nabla^{2} \mathcal{L}, s^{*}+2 \widetilde{s}\right)-\zeta_{+}}{\rho_{-}\left(\nabla^{2} \mathcal{L}, s^{*}+2 \widetilde{s}\right)-\zeta_{-}}=\frac{\rho_{+}\left(\nabla^{2} \mathcal{L}, C s^{*}\right)-\zeta_{+}}{\rho_{-}\left(\nabla^{2} \mathcal{L}, C s^{*}\right)-\zeta_{-}} & =\frac{\rho_{+}\left(\nabla^{2} \mathcal{L}, s\right)-\zeta_{+}}{\rho_{-}\left(\nabla^{2} \mathcal{L}, s\right)-\zeta_{-}} \\
& =\frac{C^{\prime}}{C^{\prime \prime}-C^{\prime \prime} / 2}=\frac{2 C^{\prime}}{C^{\prime \prime}}
\end{aligned}
$$

Since $\widetilde{s}=(C-1) / 2 \cdot s^{*}$ where $C$ satisfies (C.3), we have

$$
\widetilde{s} \geq\left(144 \cdot\left(\frac{2 C^{\prime}}{C^{\prime \prime}}\right)^{2}+250 \cdot\left(\frac{2 C^{\prime}}{C^{\prime \prime}}\right)\right) \cdot s^{*}=\left(144 \kappa^{2}+250 \kappa\right) \cdot s^{*} .
$$

Thus we find an $\widetilde{s}$ that satisfies the requirements in Assumption 4.4. Therefore, Assumption 4.4 in Wang et al. (2014a) holds with probability at least 1 $4 d^{-1}-6 d^{-2}$.

Logistic Loss: Remind that, the Hessian matrix of logistic loss is defined in (4.2) of Wang et al. (2014a), while its sparse eigenvalues $\rho_{-}\left(\nabla^{2} \mathcal{L}, s, R\right)$ and $\rho_{+}\left(\nabla^{2} \mathcal{L}, s, R\right)$ are defined in Definition 4.3 of Wang et al. (2014a), where $R \in(0,+\infty)$ is an absolute constant.

In the sequel, we show that Assumption 4.4 is a weaker assumption than the assumption of restricted strong convexity and smoothness imposed by Loh and Wainwright (2013). Since they proved that their assumption holds with high probability, Assumption 4.4 also holds with high probability.

In fact, it suffices to show if the assumption of restricted strong convexity and smoothness holds, then for a sufficiently large $n$, there exists an $s$ such that $\rho_{-}\left(\nabla^{2} \mathcal{L}, s, R\right)>0$ and $\rho_{+}\left(\nabla^{2} \mathcal{L}, s, R\right)<+\infty$. With this claim, we can justify Assumption 4.4 following the same argument as after Lemma C.3. Now we prove the previous claim.

Loh and Wainwright (2013) imposed the following assumption: For $\boldsymbol{\beta}, \boldsymbol{\beta}^{\prime} \in$ 
$\mathbb{R}^{d}$ such that $\|\boldsymbol{\beta}\|_{2} \leq R$ and $\left\|\boldsymbol{\beta}^{\prime}\right\|_{2} \leq R, \mathcal{L}(\boldsymbol{\beta})$ satisfies

$$
\begin{aligned}
& \mathcal{L}\left(\boldsymbol{\beta}^{\prime}\right)-\mathcal{L}(\boldsymbol{\beta})-\nabla \mathcal{L}(\boldsymbol{\beta})^{T}\left(\boldsymbol{\beta}^{\prime}-\boldsymbol{\beta}\right) \leq C\left\|\boldsymbol{\beta}-\boldsymbol{\beta}^{\prime}\right\|_{2}^{2}+C^{\prime} \cdot \frac{\log d}{n}\left\|\boldsymbol{\beta}-\boldsymbol{\beta}^{\prime}\right\|_{1}^{2} \\
& \mathcal{L}\left(\boldsymbol{\beta}^{\prime}\right)-\mathcal{L}(\boldsymbol{\beta})-\nabla \mathcal{L}(\boldsymbol{\beta})^{T}\left(\boldsymbol{\beta}^{\prime}-\boldsymbol{\beta}\right) \geq C^{\prime \prime}\left\|\boldsymbol{\beta}-\boldsymbol{\beta}^{\prime}\right\|_{2}^{2}-C^{\prime \prime \prime} \cdot \frac{\log d}{n}\left\|\boldsymbol{\beta}-\boldsymbol{\beta}^{\prime}\right\|_{1}^{2}
\end{aligned}
$$

Here all the constants are positive. See equations (28) and (29) of their paper for details.

By Taylor's theorem and the mean value theorem, we have

$$
\mathcal{L}\left(\boldsymbol{\beta}^{\prime}\right)=\mathcal{L}(\boldsymbol{\beta})+\nabla \mathcal{L}(\boldsymbol{\beta})^{T}\left(\boldsymbol{\beta}^{\prime}-\boldsymbol{\beta}\right)+\frac{1}{2}\left(\boldsymbol{\beta}^{\prime}-\boldsymbol{\beta}\right)^{T} \nabla^{2} \mathcal{L}\left(\gamma \boldsymbol{\beta}^{\prime}+(1-\gamma) \boldsymbol{\beta}\right)\left(\boldsymbol{\beta}^{\prime}-\boldsymbol{\beta}\right)
$$

for some $\gamma \in[0,1]$. Plugging this into the left-hand sides of (C.4) and (C.5), we have

$\frac{1}{2}\left(\boldsymbol{\beta}^{\prime}-\boldsymbol{\beta}\right)^{T} \nabla^{2} \mathcal{L}\left(\gamma \boldsymbol{\beta}^{\prime}+(1-\gamma) \boldsymbol{\beta}\right)\left(\boldsymbol{\beta}^{\prime}-\boldsymbol{\beta}\right) \leq C\left\|\boldsymbol{\beta}^{\prime}-\boldsymbol{\beta}\right\|_{2}^{2}+C^{\prime} \cdot \frac{\log d}{n}\left\|\boldsymbol{\beta}^{\prime}-\boldsymbol{\beta}\right\|_{1}^{2}$,

$$
\frac{1}{2}\left(\boldsymbol{\beta}^{\prime}-\boldsymbol{\beta}\right)^{T} \nabla^{2} \mathcal{L}\left(\gamma \boldsymbol{\beta}^{\prime}+(1-\gamma) \boldsymbol{\beta}\right)\left(\boldsymbol{\beta}^{\prime}-\boldsymbol{\beta}\right) \geq C^{\prime \prime}\left\|\boldsymbol{\beta}^{\prime}-\boldsymbol{\beta}\right\|_{2}^{2}-C^{\prime \prime \prime} \cdot \frac{\log d}{n}\left\|\boldsymbol{\beta}^{\prime}-\boldsymbol{\beta}\right\|_{1}^{2} .
$$

Suppose $\boldsymbol{\beta}$ and $\boldsymbol{\beta}^{\prime}$ satisfy $\left\|\boldsymbol{\beta}^{\prime}-\boldsymbol{\beta}\right\|_{0} \leq s$, which implies $\left\|\boldsymbol{\beta}^{\prime}-\boldsymbol{\beta}\right\|_{1} \leq \sqrt{s}$. $\left\|\boldsymbol{\beta}^{\prime}-\boldsymbol{\beta}\right\|_{2}$. Plugging this upper bound of $\left\|\boldsymbol{\beta}^{\prime}-\boldsymbol{\beta}\right\|_{1}$ into the right-hand sides of (C.6) and (C.7), we have

$$
\frac{1}{2}\left(\boldsymbol{\beta}^{\prime}-\boldsymbol{\beta}\right)^{T} \nabla^{2} \mathcal{L}\left(\gamma \boldsymbol{\beta}^{\prime}+(1-\gamma) \boldsymbol{\beta}\right)\left(\boldsymbol{\beta}^{\prime}-\boldsymbol{\beta}\right) \leq\left(C+C^{\prime} \cdot \frac{s \log d}{n}\right) \cdot\left\|\boldsymbol{\beta}^{\prime}-\boldsymbol{\beta}\right\|_{2}^{2},
$$

$$
\frac{1}{2}\left(\boldsymbol{\beta}^{\prime}-\boldsymbol{\beta}\right)^{T} \nabla^{2} \mathcal{L}\left(\gamma \boldsymbol{\beta}^{\prime}+(1-\gamma) \boldsymbol{\beta}\right)\left(\boldsymbol{\beta}^{\prime}-\boldsymbol{\beta}\right) \geq\left(C^{\prime \prime}-C^{\prime \prime \prime} \cdot \frac{s \log d}{n}\right) \cdot\left\|\boldsymbol{\beta}^{\prime}-\boldsymbol{\beta}\right\|_{2}^{2} .
$$

In (C.8) and (C.9), taking $n \geq \max \left\{2 C^{\prime \prime \prime} / C^{\prime \prime}, 2 C^{\prime} / C\right\} \cdot s \log d / n$, and dividing $\left\|\boldsymbol{\beta}^{\prime}-\boldsymbol{\beta}\right\|_{2}^{2}$ on both sides, we obtain

$$
\frac{C^{\prime \prime}}{2} \leq \frac{1}{2} \cdot \frac{\left(\boldsymbol{\beta}^{\prime}-\boldsymbol{\beta}\right)^{T}}{\left\|\boldsymbol{\beta}^{\prime}-\boldsymbol{\beta}\right\|_{2}} \cdot \nabla^{2} \mathcal{L}\left(\gamma \boldsymbol{\beta}^{\prime}+(1-\gamma) \boldsymbol{\beta}\right) \cdot \frac{\left(\boldsymbol{\beta}^{\prime}-\boldsymbol{\beta}\right)}{\left\|\boldsymbol{\beta}^{\prime}-\boldsymbol{\beta}\right\|_{2}} \leq \frac{3 C}{2} .
$$

Let $\boldsymbol{v}=\left(\boldsymbol{\beta}^{\prime}-\boldsymbol{\beta}\right) /\left\|\boldsymbol{\beta}^{\prime}-\boldsymbol{\beta}\right\|_{2}$. Obviously, $\boldsymbol{v}$ is an arbitrary vector that satisfies $\|\boldsymbol{v}\|_{2}=1$ and $\|\boldsymbol{v}\|_{0} \leq s$. Taking $\boldsymbol{\beta}^{\prime} \rightarrow \boldsymbol{\beta}$, we have $C^{\prime \prime} \leq \boldsymbol{v}^{T} \nabla^{2} \mathcal{L}(\boldsymbol{\beta}) \boldsymbol{v} \leq 3 C$ for any $\boldsymbol{\beta} \leq R$ and any $\boldsymbol{v}$ such that $\|\boldsymbol{v}\|_{2}=1$ and $\|\boldsymbol{v}\|_{0} \leq s$. By Definition 4.3 , we have $\rho_{-}\left(\nabla^{2} \mathcal{L}, s, R\right) \geq C^{\prime}>0$ and $\rho_{+}\left(\nabla^{2} \mathcal{L}, s, R\right) \leq 3 C<+\infty$.

Therefore, if their assumption holds, then for a sufficiently large $n$, there exists some $s$ such that $\rho_{-}\left(\nabla^{2} \mathcal{L}, s, R\right)>0$ and $\rho_{+}\left(\nabla^{2} \mathcal{L}, s, R\right)<+\infty$. Following the same argument as after Lemma C.3, we can show that Assumption 
4.4 also holds true. That is to say, Assumption 4.4 of Wang et al. (2014a) is a weaker assumption.

Because their assumption holds with high probability for generalized linear models (not including Poisson model; see Proposition 1 of their paper) with sub-Gaussian design, our Assumption 4.4 holds with high probability in the same setting, including logistic loss as a special case in our paper.

\section{APPENDIX D: PROOF OF THEORETICAL RESULTS}

To analyze the computational properties of our approximate regularization path following method, we first provide several useful lemmas on the proximalgradient method that is used within each stage of the path following method.

D.1. Preliminary Results about Proximal-Gradient Method. Recall that the objective function can be formulated as $\phi_{\lambda_{t}}(\boldsymbol{\beta})=\widetilde{\mathcal{L}}_{\lambda_{t}}(\boldsymbol{\beta})+\lambda_{t}\|\boldsymbol{\beta}\|_{1}$ where $\widetilde{\mathcal{L}}_{\lambda_{t}}(\boldsymbol{\beta})=\mathcal{L}(\boldsymbol{\beta})+\mathcal{Q}_{\lambda_{t}}(\boldsymbol{\beta})$, while $\psi_{L_{t}^{k}, \lambda_{t}}\left(\boldsymbol{\beta} ; \boldsymbol{\beta}_{t}^{k-1}\right)$ is the local quadratic approximation of $\phi_{\lambda_{t}}(\boldsymbol{\beta})$ at $\boldsymbol{\beta}_{t}^{k-1}$, as defined in (3.7) of Wang et al. (2014a). The following lemma, which is adapted from Nesterov (2013), characterizes the decrement of the objective function.

Lemma D.1. Under Assumption 4.4, we assume $\left\|\left(\boldsymbol{\beta}_{t}^{k-1}\right)_{\bar{S}^{*}}\right\|_{0} \leq \widetilde{s}$, where $\widetilde{s}$ is the positive integer specified in Assumption 4.4. For any $L_{t}^{k}>0$ and fixed $\lambda_{t} \in\left[\lambda_{\text {tgt }}, \lambda_{0}\right]$, we have

$$
\phi_{\lambda_{t}}\left(\boldsymbol{\beta}_{t}^{k}\right) \leq \phi_{\lambda_{t}}\left(\boldsymbol{\beta}_{t}^{k-1}\right)-\frac{L_{t}^{k}}{2}\left\|\boldsymbol{\beta}_{t}^{k}-\boldsymbol{\beta}_{t}^{k-1}\right\|_{2}^{2}
$$

Recall that, as defined in (3.15) of Wang et al. (2014a), $\omega_{\lambda}(\boldsymbol{\beta})$ characterizes the suboptimality of approximate solutions. The next lemma, which is also adapted from Nesterov (2013), provides an upper bound of $\omega_{\lambda_{t}}\left(\boldsymbol{\beta}_{t}^{k}\right)$ using $\left\|\boldsymbol{\beta}_{t}^{k}-\boldsymbol{\beta}_{t}^{k-1}\right\|_{2}$.

Lemma D.2. Under the assumptions of Lemma D.1, then we have

$$
\omega_{\lambda_{t}}\left(\boldsymbol{\beta}_{t}^{k}\right) \leq\left(L_{t}^{k}+\rho_{+}-\zeta_{-}\right)\left\|\boldsymbol{\beta}_{t}^{k}-\boldsymbol{\beta}_{t}^{k-1}\right\|_{2},
$$

where $\rho_{+}=\rho_{+}\left(\nabla^{2} \mathcal{L}, s^{*}+2 \widetilde{s}\right)$ is the sparse eigenvalue specified in Assumption 4.4; As defined in regularity condition (a), $\zeta_{+}>0$ is the concavity parameter of the nonconvex penalty, which satisfies (4.6) in Wang et al. (2014a).

\section{D.2. Proof of Lemma 5.1 in Wang et al. (2014a).}

Proof. Recall that $\mathcal{Q}_{\lambda}(\boldsymbol{\beta})$ is the concave component of the nonconvex penalty $\mathcal{P}_{\lambda}(\boldsymbol{\beta})$, which implies $-\mathcal{Q}_{\lambda}(\boldsymbol{\beta})$ is convex. Meanwhile, recall that 
$\mathcal{Q}_{\lambda}(\boldsymbol{\beta})=\sum_{j=1}^{d} q_{\lambda}\left(\beta_{j}\right)$, where $q_{\lambda}\left(\beta_{j}\right)$ satisfies regularity condition (a) in Wang et al. (2014a). Hence we have

$$
-\zeta_{-}\left(\beta_{j}^{\prime}-\beta_{j}\right)^{2} \leq\left(q_{\lambda}^{\prime}\left(\beta_{j}^{\prime}\right)-q_{\lambda}^{\prime}\left(\beta_{j}\right)\right)\left(\beta_{j}^{\prime}-\beta_{j}\right) \leq-\zeta_{+}\left(\beta_{j}^{\prime}-\beta_{j}\right)^{2},
$$

which implies the convex function $-\mathcal{Q}_{\lambda}(\boldsymbol{\beta})$ satisfies

$$
\begin{aligned}
& \left(\nabla\left(-\mathcal{Q}_{\lambda}\left(\boldsymbol{\beta}^{\prime}\right)\right)-\nabla\left(-\mathcal{Q}_{\lambda}(\boldsymbol{\beta})\right)\right)^{T}\left(\boldsymbol{\beta}^{\prime}-\boldsymbol{\beta}\right) \leq \zeta_{-}\left\|\boldsymbol{\beta}^{\prime}-\boldsymbol{\beta}\right\|_{2}^{2}, \\
& \left(\nabla\left(-\mathcal{Q}_{\lambda}\left(\boldsymbol{\beta}^{\prime}\right)\right)-\nabla\left(-\mathcal{Q}_{\lambda}(\boldsymbol{\beta})\right)\right)^{T}\left(\boldsymbol{\beta}^{\prime}-\boldsymbol{\beta}\right) \geq \zeta_{+}\left\|\boldsymbol{\beta}^{\prime}-\boldsymbol{\beta}\right\|_{2}^{2} .
\end{aligned}
$$

According to Nesterov (2004, Theorem 2.1.5 \& Theorem 2.1.9), (D.1) and (D.2) are equivalent definitions of strong smoothness and strong convexity respectively. In other words, $-\mathcal{Q}_{\lambda}(\boldsymbol{\beta})$ satisfies

$$
\begin{aligned}
& -\mathcal{Q}_{\lambda}\left(\boldsymbol{\beta}^{\prime}\right) \leq-\mathcal{Q}_{\lambda}(\boldsymbol{\beta})-\nabla \mathcal{Q}(\boldsymbol{\beta})^{T}\left(\boldsymbol{\beta}^{\prime}-\boldsymbol{\beta}\right)+\frac{\zeta_{-}}{2}\left\|\boldsymbol{\beta}^{\prime}-\boldsymbol{\beta}\right\|_{2}^{2}, \\
& -\mathcal{Q}_{\lambda}\left(\boldsymbol{\beta}^{\prime}\right) \geq-\mathcal{Q}_{\lambda}(\boldsymbol{\beta})-\nabla \mathcal{Q}(\boldsymbol{\beta})^{T}\left(\boldsymbol{\beta}^{\prime}-\boldsymbol{\beta}\right)+\frac{\zeta_{+}}{2}\left\|\boldsymbol{\beta}^{\prime}-\boldsymbol{\beta}\right\|_{2}^{2} .
\end{aligned}
$$

For loss function $\mathcal{L}(\boldsymbol{\beta})$, by Taylor's theorem and the mean value theorem, we have

$$
\begin{aligned}
\mathcal{L}\left(\boldsymbol{\beta}^{\prime}\right)=\mathcal{L}(\boldsymbol{\beta}) & +\nabla \mathcal{L}(\boldsymbol{\beta})^{T}\left(\boldsymbol{\beta}^{\prime}-\boldsymbol{\beta}\right) \\
& +\frac{1}{2}\left(\boldsymbol{\beta}^{\prime}-\boldsymbol{\beta}\right)^{T} \nabla^{2} \mathcal{L}\left(\gamma \boldsymbol{\beta}+(1-\gamma) \boldsymbol{\beta}^{\prime}\right)\left(\boldsymbol{\beta}^{\prime}-\boldsymbol{\beta}\right),
\end{aligned}
$$

where $\gamma \in[0,1]$. Note that we assume $\left\|\left(\boldsymbol{\beta}^{\prime}-\boldsymbol{\beta}\right)_{\overline{S^{*}}}\right\|_{0} \leq 2 \widetilde{s}$, which implies $\left\|\boldsymbol{\beta}^{\prime}-\boldsymbol{\beta}\right\|_{0} \leq s^{*}+2 \widetilde{s}$. For logistic loss, we assume $\|\boldsymbol{\beta}\|_{2} \leq R$ and $\left\|\boldsymbol{\beta}^{\prime}\right\|_{2} \leq R$, which implies $\left\|\gamma \boldsymbol{\beta}+(1-\gamma) \boldsymbol{\beta}^{\prime}\right\|_{2} \leq R$ by the convexity of $\ell_{2}$ norm. Hence, by Definition 4.2 and Definition 4.3 of Wang et al. (2014a), we have

$\frac{\left(\boldsymbol{\beta}^{\prime}-\boldsymbol{\beta}\right)^{T}}{\left\|\boldsymbol{\beta}^{\prime}-\boldsymbol{\beta}\right\|_{2}} \nabla^{2} \mathcal{L}\left(\gamma \boldsymbol{\beta}+(1-\gamma) \boldsymbol{\beta}^{\prime}\right) \frac{\left(\boldsymbol{\beta}^{\prime}-\boldsymbol{\beta}\right)}{\left\|\boldsymbol{\beta}^{\prime}-\boldsymbol{\beta}\right\|_{2}} \in\left[\rho_{+}\left(\nabla^{2} \mathcal{L}, s^{*}+2 \widetilde{s}\right), \rho_{-}\left(\nabla^{2} \mathcal{L}, s^{*}+2 \widetilde{s}\right)\right]$.

Plugging this into the right-hand side of (D.5), we have

$$
\begin{aligned}
& \mathcal{L}\left(\boldsymbol{\beta}^{\prime}\right) \geq \mathcal{L}(\boldsymbol{\beta})+\nabla \mathcal{L}(\boldsymbol{\beta})^{T}\left(\boldsymbol{\beta}^{\prime}-\boldsymbol{\beta}\right)+\frac{\rho_{-}\left(\nabla^{2} \mathcal{L}, s^{*}+2 \widetilde{s}\right)}{2}\left\|\boldsymbol{\beta}^{\prime}-\boldsymbol{\beta}\right\|_{2}^{2}, \\
& \mathcal{L}\left(\boldsymbol{\beta}^{\prime}\right) \leq \mathcal{L}(\boldsymbol{\beta})+\nabla \mathcal{L}(\boldsymbol{\beta})^{T}\left(\boldsymbol{\beta}^{\prime}-\boldsymbol{\beta}\right)+\frac{\rho_{+}\left(\nabla^{2} \mathcal{L}, s^{*}+2 \widetilde{s}\right)}{2}\left\|\boldsymbol{\beta}^{\prime}-\boldsymbol{\beta}\right\|_{2}^{2} .
\end{aligned}
$$

Recall that $\widetilde{\mathcal{L}}_{\lambda}(\boldsymbol{\beta})=\mathcal{L}(\boldsymbol{\beta})+\mathcal{Q}_{\lambda}(\boldsymbol{\beta})$. Subtracting (D.3) from (D.6), and (D.4) 
from (D.7), we obtain

$$
\begin{aligned}
& \widetilde{\mathcal{L}}_{\lambda}\left(\boldsymbol{\beta}^{\prime}\right) \geq \widetilde{\mathcal{L}}_{\lambda}(\boldsymbol{\beta})+\nabla \widetilde{\mathcal{L}}_{\lambda}(\boldsymbol{\beta})^{T}\left(\boldsymbol{\beta}^{\prime}-\boldsymbol{\beta}\right)+\frac{\rho_{-}\left(\nabla^{2} \mathcal{L}, s^{*}+2 \widetilde{s}\right)-\zeta_{-}}{2}\left\|\boldsymbol{\beta}^{\prime}-\boldsymbol{\beta}\right\|_{2}^{2} \\
& \widetilde{\mathcal{L}}_{\lambda}\left(\boldsymbol{\beta}^{\prime}\right) \leq \widetilde{\mathcal{L}}_{\lambda}(\boldsymbol{\beta})+\nabla \widetilde{\mathcal{L}}_{\lambda}(\boldsymbol{\beta})^{T}\left(\boldsymbol{\beta}^{\prime}-\boldsymbol{\beta}\right)+\frac{\rho_{+}\left(\nabla^{2} \mathcal{L}, s^{*}+2 \widetilde{s}\right)-\zeta_{+}}{2}\left\|\boldsymbol{\beta}^{\prime}-\boldsymbol{\beta}\right\|_{2}^{2}
\end{aligned}
$$

Then we conclude the proof.

\section{D.3. Proof of Lemma 5.2 in Wang et al. (2014a).}

Proof. Statistical Recovery: Since $\left\|\boldsymbol{\beta}_{\overline{S^{*}}}\right\|_{0} \leq \widetilde{s}$ and $\left\|\boldsymbol{\beta}_{\overline{S^{*}}}^{*}\right\|_{0}=0$, we have $\left\|\left(\boldsymbol{\beta}-\boldsymbol{\beta}^{*}\right)_{\overline{S^{*}}}\right\| \leq \widetilde{s}$. For logistic loss, we further have $\|\tilde{\boldsymbol{\beta}}\|_{2} \leq R$ and $\left\|\boldsymbol{\beta}^{*}\right\|_{2} \leq R$. Thus Lemma 5.1 of Wang et al. (2014a) gives

$$
\begin{aligned}
& \widetilde{\mathcal{L}}_{\lambda}\left(\boldsymbol{\beta}^{*}\right) \geq \widetilde{\mathcal{L}}_{\lambda}(\boldsymbol{\beta})+\left(\boldsymbol{\beta}^{*}-\boldsymbol{\beta}\right)^{T} \nabla \widetilde{\mathcal{L}}_{\lambda}(\boldsymbol{\beta})+\frac{\rho_{-}-\zeta_{-}}{2}\left\|\boldsymbol{\beta}^{*}-\boldsymbol{\beta}\right\|_{2}^{2}, \\
& \widetilde{\mathcal{L}}_{\lambda}(\boldsymbol{\beta}) \geq \widetilde{\mathcal{L}}_{\lambda}\left(\boldsymbol{\beta}^{*}\right)+\left(\boldsymbol{\beta}-\boldsymbol{\beta}^{*}\right)^{T} \nabla \widetilde{\mathcal{L}}_{\lambda}\left(\boldsymbol{\beta}^{*}\right)+\frac{\rho_{-}-\zeta_{-}}{2}\left\|\boldsymbol{\beta}^{*}-\boldsymbol{\beta}\right\|_{2}^{2} .
\end{aligned}
$$

Adding (D.8) and (D.9) and moving $\left(\boldsymbol{\beta}^{*}-\boldsymbol{\beta}\right)^{T} \nabla \widetilde{\mathcal{L}}_{\lambda}(\boldsymbol{\beta})$ to the left-hand side, we obtain

(D.10) $\quad\left(\boldsymbol{\beta}-\boldsymbol{\beta}^{*}\right)^{T} \nabla \widetilde{\mathcal{L}}_{\lambda}(\boldsymbol{\beta}) \geq\left(\boldsymbol{\beta}-\boldsymbol{\beta}^{*}\right)^{T} \nabla \widetilde{\mathcal{L}}_{\lambda}\left(\boldsymbol{\beta}^{*}\right)+\left(\rho_{-}-\zeta_{-}\right)\left\|\boldsymbol{\beta}^{*}-\boldsymbol{\beta}\right\|_{2}^{2}$.

Let $\boldsymbol{\xi} \in \partial\|\boldsymbol{\beta}\|_{1}$ be the subgradient that attains the minimum in

$$
\omega_{\lambda}(\boldsymbol{\beta})=\min _{\boldsymbol{\xi}^{\prime} \in \partial\|\boldsymbol{\beta}\|_{1}} \max _{\boldsymbol{\beta}^{\prime} \in \Omega}\left\{\frac{\left(\boldsymbol{\beta}-\boldsymbol{\beta}^{\prime}\right)^{T}}{\left\|\boldsymbol{\beta}-\boldsymbol{\beta}^{\prime}\right\|_{1}}\left(\nabla \widetilde{\mathcal{L}}_{\lambda}(\boldsymbol{\beta})+\lambda \boldsymbol{\xi}^{\prime}\right)\right\} .
$$

Then we have

$$
\omega_{\lambda}(\boldsymbol{\beta})=\max _{\boldsymbol{\beta}^{\prime} \in \Omega}\left\{\frac{\left(\boldsymbol{\beta}-\boldsymbol{\beta}^{\prime}\right)^{T}}{\left\|\boldsymbol{\beta}-\boldsymbol{\beta}^{\prime}\right\|_{1}}\left(\nabla \widetilde{\mathcal{L}}_{\lambda}(\boldsymbol{\beta})+\lambda \boldsymbol{\xi}\right)\right\} .
$$

Adding $\lambda\left(\boldsymbol{\beta}-\boldsymbol{\beta}^{*}\right)^{T} \boldsymbol{\xi}$ to the both sides of (D.10), we obtain

$$
\begin{aligned}
& \left(\boldsymbol{\beta}-\boldsymbol{\beta}^{*}\right)^{T}\left(\nabla \widetilde{\mathcal{L}}_{\lambda}(\boldsymbol{\beta})+\lambda \boldsymbol{\xi}\right) \\
& \geq\left(\boldsymbol{\beta}-\boldsymbol{\beta}^{*}\right)^{T} \nabla \widetilde{\mathcal{L}}_{\lambda}\left(\boldsymbol{\beta}^{*}\right)+\left(\rho_{-}-\zeta_{-}\right)\left\|\boldsymbol{\beta}^{*}-\boldsymbol{\beta}\right\|_{2}^{2}+\lambda\left(\boldsymbol{\beta}-\boldsymbol{\beta}^{*}\right)^{T} \boldsymbol{\xi} .
\end{aligned}
$$

Since $\boldsymbol{\beta}^{*} \in \Omega$, by (D.11) we have

$$
\begin{aligned}
\frac{\left(\boldsymbol{\beta}-\boldsymbol{\beta}^{*}\right)^{T}}{\left\|\boldsymbol{\beta}-\boldsymbol{\beta}^{*}\right\|_{1}}\left(\nabla \widetilde{\mathcal{L}}_{\lambda}(\boldsymbol{\beta})+\lambda \boldsymbol{\xi}\right) & \leq \max _{\boldsymbol{\beta}^{\prime} \in \Omega}\left\{\frac{\left(\boldsymbol{\beta}-\boldsymbol{\beta}^{\prime}\right)^{T}}{\left\|\boldsymbol{\beta}-\boldsymbol{\beta}^{\prime}\right\|_{1}}\left(\nabla \widetilde{\mathcal{L}}_{\lambda}(\boldsymbol{\beta})+\lambda \boldsymbol{\xi}\right)\right\} \\
& =\omega_{\lambda}(\boldsymbol{\beta}) .
\end{aligned}
$$

Recall that we assume $\omega_{\lambda}(\boldsymbol{\beta}) \leq \lambda / 2$, we obtain

$$
\left(\boldsymbol{\beta}-\boldsymbol{\beta}^{*}\right)^{T}\left(\nabla \widetilde{\mathcal{L}}_{\lambda}(\boldsymbol{\beta})+\lambda \boldsymbol{\xi}\right) \leq \lambda / 2 \cdot\left\|\boldsymbol{\beta}-\boldsymbol{\beta}^{*}\right\|_{1} .
$$


Plugging (D.13) into the left-hand side of (D.10), we obtain

$$
\begin{aligned}
& \lambda / 2 \cdot\left\|\boldsymbol{\beta}-\boldsymbol{\beta}^{*}\right\|_{1} \\
& \geq \underbrace{\left(\boldsymbol{\beta}-\boldsymbol{\beta}^{*}\right)^{T} \nabla \widetilde{\mathcal{L}}_{\lambda}\left(\boldsymbol{\beta}^{*}\right)}_{(\mathrm{i})}+\left(\rho_{-}-\zeta_{-}\right)\left\|\boldsymbol{\beta}^{*}-\boldsymbol{\beta}\right\|_{2}^{2}+\underbrace{\lambda\left(\boldsymbol{\beta}-\boldsymbol{\beta}^{*}\right)^{T} \boldsymbol{\xi}}_{(\mathrm{ii})} .
\end{aligned}
$$

Now we provide lower bounds of terms (i) and (ii) in (D.14) respectively.

- Bounding Term (i) in (D.14): Recall that $\widetilde{\mathcal{L}}_{\lambda}(\boldsymbol{\beta})=\mathcal{L}(\boldsymbol{\beta})+\mathcal{Q}_{\lambda}(\boldsymbol{\beta})$. We have

$$
\left(\boldsymbol{\beta}-\boldsymbol{\beta}^{*}\right)^{T} \nabla \widetilde{\mathcal{L}}_{\lambda}\left(\boldsymbol{\beta}^{*}\right)=\underbrace{\left(\boldsymbol{\beta}-\boldsymbol{\beta}^{*}\right)^{T} \nabla \mathcal{L}\left(\boldsymbol{\beta}^{*}\right)}_{\text {(i).a }}+\underbrace{\left(\boldsymbol{\beta}-\boldsymbol{\beta}^{*}\right)^{T} \nabla \mathcal{Q}_{\lambda}\left(\boldsymbol{\beta}^{*}\right)}_{\text {(i).b }} .
$$

Separating the support of $\boldsymbol{\beta}-\boldsymbol{\beta}^{*}$ into $S^{*}$ and $\overline{S^{*}}$, we obtain

$$
\left\|\boldsymbol{\beta}-\boldsymbol{\beta}^{*}\right\|_{1}=\left\|\left(\boldsymbol{\beta}-\boldsymbol{\beta}^{*}\right)_{\overline{S^{*}}}\right\|_{1}+\left\|\left(\boldsymbol{\beta}-\boldsymbol{\beta}^{*}\right)_{S^{*}}\right\|_{1} .
$$

Then for term (i).a in (D.15), we have

$$
\begin{aligned}
& \left(\boldsymbol{\beta}-\boldsymbol{\beta}^{*}\right)^{T} \nabla \mathcal{L}\left(\boldsymbol{\beta}^{*}\right) \\
& \geq-\left\|\boldsymbol{\beta}-\boldsymbol{\beta}^{*}\right\|_{1}\left\|\nabla \mathcal{L}\left(\boldsymbol{\beta}^{*}\right)\right\|_{\infty} \\
(\mathrm{D} .16) \quad & =-\left\|\left(\boldsymbol{\beta}-\boldsymbol{\beta}^{*}\right)_{S^{*}}\right\|_{1}\left\|\nabla \mathcal{L}\left(\boldsymbol{\beta}^{*}\right)\right\|_{\infty}-\left\|\left(\boldsymbol{\beta}-\boldsymbol{\beta}^{*}\right)_{S^{*}}\right\|_{1}\left\|\nabla \mathcal{L}\left(\boldsymbol{\beta}^{*}\right)\right\|_{\infty} .
\end{aligned}
$$

For term (i).b in (D.15), we have

$$
\begin{aligned}
& \left(\boldsymbol{\beta}-\boldsymbol{\beta}^{*}\right)^{T} \nabla \mathcal{Q}_{\lambda}\left(\boldsymbol{\beta}^{*}\right) \\
& =\left(\boldsymbol{\beta}-\boldsymbol{\beta}^{*}\right)_{S^{*}}^{T}\left(\nabla \mathcal{Q}_{\lambda}\left(\boldsymbol{\beta}^{*}\right)\right)_{S^{*}}+\left(\boldsymbol{\beta}-\boldsymbol{\beta}^{*}\right) \frac{T}{S^{*}}\left(\nabla \mathcal{Q}_{\lambda}\left(\boldsymbol{\beta}^{*}\right)\right)_{\overline{S^{*}}} .
\end{aligned}
$$

Note that $\mathcal{Q}_{\lambda}\left(\boldsymbol{\beta}^{*}\right)$ is separable. We have

$$
\begin{aligned}
\left(\boldsymbol{\beta}-\boldsymbol{\beta}^{*}\right)_{S^{*}}^{T}\left(\nabla \mathcal{Q}_{\lambda}\left(\boldsymbol{\beta}^{*}\right)\right)_{S^{*}} & =\sum_{j \in S^{*}}\left(\beta_{j}-\beta_{j}^{*}\right) \cdot q_{\lambda}^{\prime}\left(\beta_{j}^{*}\right) \\
& =\left(\boldsymbol{\beta}-\boldsymbol{\beta}^{*}\right)_{S^{*}}^{T} \nabla \mathcal{Q}_{\lambda}\left(\boldsymbol{\beta}^{*}\right), \\
\left(\boldsymbol{\beta}-\boldsymbol{\beta}^{*}\right)_{\overline{S^{*}}}^{T}\left(\nabla \mathcal{Q}_{\lambda}\left(\boldsymbol{\beta}^{*}\right)\right)_{\overline{S^{*}}} & =\sum_{j \in \overline{S^{*}}}\left(\beta_{j}-\beta_{j}^{*}\right) \cdot q_{\lambda}^{\prime}\left(\beta_{j}^{*}\right) \\
& =\sum_{j \in \overline{S^{*}}}\left(\beta_{j}-\beta_{j}^{*}\right) \cdot q_{\lambda}^{\prime}(0)=0,
\end{aligned}
$$

where the second equation in (D.19) is because $\beta_{j}^{*}=0$ for $j \in \overline{S^{*}}$, and the third is by regularity condition (c) that $q_{\lambda}^{\prime}(0)=0$. Plugging (D.18) and (D.19) into the right-hand side of (D.17), for term (i).b in (D.15) 
we obtain

$$
\begin{aligned}
\left(\boldsymbol{\beta}-\boldsymbol{\beta}^{*}\right)^{T} \nabla \mathcal{Q}_{\lambda}\left(\boldsymbol{\beta}^{*}\right) & =\left(\boldsymbol{\beta}-\boldsymbol{\beta}^{*}\right)_{S^{*}}^{T} \nabla \mathcal{Q}_{\lambda}\left(\boldsymbol{\beta}^{*}\right) \\
& \geq-\left\|\left(\boldsymbol{\beta}-\boldsymbol{\beta}^{*}\right)_{S^{*}}\right\|_{1}\left\|\nabla \mathcal{Q}_{\lambda}\left(\boldsymbol{\beta}^{*}\right)\right\|_{\infty}
\end{aligned}
$$

Plugging (D.16) and (D.20) into the right-hand side of (D.15), then for term (i) in (D.14) we obtain

$$
\begin{gathered}
\left(\boldsymbol{\beta}-\boldsymbol{\beta}^{*}\right)^{T} \nabla \widetilde{\mathcal{L}}_{\lambda}\left(\boldsymbol{\beta}^{*}\right) \\
(\mathrm{D} .21) \quad \geq-\left\|\left(\boldsymbol{\beta}-\boldsymbol{\beta}^{*}\right)_{S^{*}}\right\|_{1}\left\|\nabla \mathcal{L}\left(\boldsymbol{\beta}^{*}\right)\right\|_{\infty}-\left\|\left(\boldsymbol{\beta}-\boldsymbol{\beta}^{*}\right)_{S^{*}}\right\|_{1}\left\|\nabla \mathcal{L}\left(\boldsymbol{\beta}^{*}\right)\right\|_{\infty} \\
-\left\|\left(\boldsymbol{\beta}-\boldsymbol{\beta}^{*}\right)_{S^{*}}\right\|_{1}\left\|\nabla \mathcal{Q}_{\lambda}\left(\boldsymbol{\beta}^{*}\right)\right\|_{\infty} .
\end{gathered}
$$

- Bounding Term (ii) in (D.14): For term (ii) in (D.14), by separating the support of $\boldsymbol{\beta}-\boldsymbol{\beta}^{*}$ into $S^{*}$ and $\overline{S^{*}}$ we have

$$
\lambda\left(\boldsymbol{\beta}-\boldsymbol{\beta}^{*}\right)^{T} \boldsymbol{\xi}=\lambda \underbrace{\left(\boldsymbol{\beta}-\boldsymbol{\beta}^{*}\right)_{S^{*}}^{T} \boldsymbol{\xi}_{S^{*}}}_{(\mathrm{ii}) . \mathrm{a}}+\lambda \underbrace{\left(\boldsymbol{\beta}-\boldsymbol{\beta}^{*}\right) \frac{T}{S^{*}} \boldsymbol{\xi}_{S^{*}}}_{(\mathrm{ii}) . \mathrm{b}} .
$$

For term (ii).a in (D.22), since $\boldsymbol{\xi} \in \partial\|\boldsymbol{\beta}\|_{1}$, we have $\left\|\boldsymbol{\xi}_{S^{*}}\right\|_{\infty} \leq\|\boldsymbol{\xi}\|_{\infty} \leq 1$, which implies

(D.23) $\left(\boldsymbol{\beta}-\boldsymbol{\beta}^{*}\right)_{S^{*}}^{T} \boldsymbol{\xi}_{S^{*}} \geq-\left\|\boldsymbol{\xi}_{S^{*}}\right\|_{\infty}\left\|\left(\boldsymbol{\beta}-\boldsymbol{\beta}^{*}\right)_{S^{*}}\right\|_{1} \geq-\left\|\left(\boldsymbol{\beta}-\boldsymbol{\beta}^{*}\right)_{S^{*}}\right\|_{1}$.

For term (ii).b in (D.22), note that $\boldsymbol{\beta}_{\overline{S^{*}}}^{*}=\mathbf{0}$. Hence, $\left(\boldsymbol{\beta}-\boldsymbol{\beta}^{*}\right) \overline{S^{*}}=\boldsymbol{\beta}_{\overline{S^{*}}}$. Recall $\boldsymbol{\xi} \in \partial\|\boldsymbol{\beta}\|_{1}$. For $\beta_{j} \neq 0$, since $\xi_{j}=\operatorname{sign}\left(\beta_{j}\right)$, we have $\beta_{j} \xi_{j}=\left|\beta_{j}\right|$. For $\beta_{j}=0$, we have $\beta_{j} \xi_{j}=\left|\beta_{j}\right|=0$. Therefore, we obtain

$$
\begin{aligned}
\left(\boldsymbol{\beta}-\boldsymbol{\beta}^{*}\right)_{\overline{S^{*}}}^{T} \boldsymbol{\xi}_{\overline{S^{*}}}=\boldsymbol{\beta}_{\overline{S^{*}}}^{T} \boldsymbol{\xi}_{\overline{S^{*}}}=\sum_{j \in \overline{S^{*}}} \beta_{j} \xi_{j}=\sum_{j \in \overline{S^{*}}}\left|\beta_{j}\right| & =\left\|\boldsymbol{\beta}_{\overline{S^{*}}}\right\|_{1} \\
& =\left\|\left(\boldsymbol{\beta}-\boldsymbol{\beta}^{*}\right)_{\overline{S^{*}}}\right\|_{1} .
\end{aligned}
$$

Plugging (D.23) and (D.24) into the right-hand side of (D.22), we obtain

$$
\lambda\left(\boldsymbol{\beta}-\boldsymbol{\beta}^{*}\right)^{T} \boldsymbol{\xi} \geq-\lambda\left\|\left(\boldsymbol{\beta}-\boldsymbol{\beta}^{*}\right)_{S^{*}}\right\|_{1}+\lambda\left\|\left(\boldsymbol{\beta}-\boldsymbol{\beta}^{*}\right)_{\overline{S^{*}}}\right\|_{1}
$$

Plugging (D.21) and (D.25) into the right-hand side of (D.14), we obtain

$$
\begin{aligned}
& \lambda / 2 \cdot\left\|\boldsymbol{\beta}-\boldsymbol{\beta}^{*}\right\|_{1}
\end{aligned}
$$

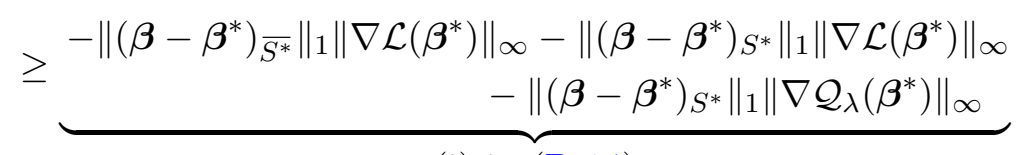

$$
\begin{aligned}
& \text { (i) in (D.14) } \\
& +\left(\rho_{-}-\zeta_{-}\right)\left\|\boldsymbol{\beta}^{*}-\boldsymbol{\beta}\right\|_{2}^{2} \underbrace{-\lambda\left\|\left(\boldsymbol{\beta}-\boldsymbol{\beta}^{*}\right)_{S^{*}}\right\|_{1}+\lambda\left\|\left(\boldsymbol{\beta}-\boldsymbol{\beta}^{*}\right)_{S^{*}}\right\|_{1}} .
\end{aligned}
$$

Again, we separate the left-hand side of (D.26) as $\lambda / 2 \cdot\left\|\boldsymbol{\beta}-\boldsymbol{\beta}^{*}\right\|_{1}=\lambda / 2$. 
$\left\|\left(\boldsymbol{\beta}-\boldsymbol{\beta}^{*}\right)_{\overline{S^{*}}}\right\|_{1}+\lambda / 2 \cdot\left\|\left(\boldsymbol{\beta}-\boldsymbol{\beta}^{*}\right)_{S^{*}}\right\|_{1}$. Rearranging the terms, we obtain

$$
\begin{aligned}
& \left(\rho_{-}-\zeta_{-}\right)\left\|\boldsymbol{\beta}-\boldsymbol{\beta}^{*}\right\|_{2}^{2}+\underbrace{\left(\lambda / 2-\left\|\nabla \mathcal{L}\left(\boldsymbol{\beta}^{*}\right)\right\|_{\infty}\right)\left\|\left(\boldsymbol{\beta}-\boldsymbol{\beta}^{*}\right)_{S^{*}}\right\|_{1}}_{(\mathrm{i})} \\
& \leq(3 \lambda / 2+\underbrace{\left\|\nabla \mathcal{L}\left(\boldsymbol{\beta}^{*}\right)\right\|_{\infty}}_{(\mathrm{ii})}+\underbrace{\left\|\nabla \mathcal{Q}_{\lambda}\left(\boldsymbol{\beta}^{*}\right)\right\|_{\infty}}_{(\mathrm{iii})})\left\|\left(\boldsymbol{\beta}-\boldsymbol{\beta}^{*}\right)_{S^{*}}\right\|_{1} .
\end{aligned}
$$

For term (ii) in (D.27), by (4.1) in Assumption 4.1 of Wang et al. (2014a) and $\lambda \geq \lambda_{\text {tgt }}$ we have

$$
\left\|\nabla \mathcal{L}\left(\boldsymbol{\beta}^{*}\right)\right\|_{\infty} \leq \lambda_{\text {tgt }} / 8 \leq \lambda / 8 .
$$

Meanwhile, (D.28) also implies that term (i) in (D.27) is positive. Recall that $\mathcal{Q}_{\lambda}(\boldsymbol{\beta})=\sum_{j=1}^{d} q_{\lambda}\left(\beta_{j}\right)$, where $q_{\lambda}\left(\beta_{j}\right)$ satisfies regularity condition (d) in Wang et al. (2014a). Hence for term (iii) in (D.27) we have

$$
\left\|\nabla \mathcal{Q}_{\lambda}\left(\boldsymbol{\beta}^{*}\right)\right\|_{\infty}=\max _{1 \leq j \leq d}\left|q_{\lambda}^{\prime}\left(\beta_{j}^{*}\right)\right| \leq \lambda .
$$

In summary, from (D.27) we obtain

$$
\begin{aligned}
\left(\rho_{-}-\zeta_{-}\right)\left\|\boldsymbol{\beta}-\boldsymbol{\beta}^{*}\right\|_{2}^{2} & \leq\left(3 \lambda / 2+\left\|\nabla \mathcal{L}\left(\boldsymbol{\beta}^{*}\right)\right\|_{\infty}+\left\|\nabla \mathcal{Q}_{\lambda}\left(\boldsymbol{\beta}^{*}\right)\right\|_{\infty}\right)\left\|\left(\boldsymbol{\beta}-\boldsymbol{\beta}^{*}\right)_{S^{*}}\right\|_{1} \\
& \leq(3 \lambda / 2+\lambda / 8+\lambda)\left\|\left(\boldsymbol{\beta}-\boldsymbol{\beta}^{*}\right)_{S^{*}}\right\|_{1} \\
& \leq 21 \lambda / 8 \cdot \sqrt{s^{*}}\left\|\left(\boldsymbol{\beta}-\boldsymbol{\beta}^{*}\right)_{S^{*}}\right\|_{2} \\
\text { (D.30) } & \leq 21 \lambda / 8 \cdot \sqrt{s^{*}}\left\|\boldsymbol{\beta}-\boldsymbol{\beta}^{*}\right\|_{2} .
\end{aligned}
$$

According to (4.3) of Wang et al. (2014a), we have $\rho_{-}-\zeta_{-}>0$. Therefore, (D.30) gives

$$
\left\|\boldsymbol{\beta}-\boldsymbol{\beta}^{*}\right\|_{2} \leq \frac{21 / 8}{\rho_{-}-\zeta_{-}} \lambda \sqrt{s^{*}}
$$

which implies the first conclusion.

Results for the Objective Function Value: Note that on the right-hand side of (D.9), we have $\rho_{-}-\zeta_{-}>0$, which gives

$$
\widetilde{\mathcal{L}}_{\lambda}\left(\boldsymbol{\beta}^{*}\right) \geq \widetilde{\mathcal{L}}_{\lambda}(\boldsymbol{\beta})+\left(\boldsymbol{\beta}^{*}-\boldsymbol{\beta}\right)^{T} \nabla \widetilde{\mathcal{L}}_{\lambda}(\boldsymbol{\beta}) .
$$

Meanwhile, since $\boldsymbol{\xi} \in \partial\|\boldsymbol{\beta}\|_{1}$, by the convexity of $\ell_{1}$ norm we have

$$
\lambda\left\|\boldsymbol{\beta}^{*}\right\|_{1} \geq \lambda\|\boldsymbol{\beta}\|_{1}+\lambda\left(\boldsymbol{\beta}^{*}-\boldsymbol{\beta}\right)^{T} \boldsymbol{\xi} .
$$

Recall that $\phi_{\lambda}(\boldsymbol{\beta})=\widetilde{\mathcal{L}}_{\lambda}(\boldsymbol{\beta})+\lambda\|\boldsymbol{\beta}\|_{1}$. Adding (D.32) and (D.33), we obtain

$$
\phi_{\lambda}\left(\boldsymbol{\beta}^{*}\right) \geq \phi_{\lambda}(\boldsymbol{\beta})+\left(\boldsymbol{\beta}^{*}-\boldsymbol{\beta}\right)^{T}\left(\nabla \widetilde{\mathcal{L}}_{\lambda}(\boldsymbol{\beta})+\lambda \boldsymbol{\xi}\right),
$$

which implies

$$
\phi_{\lambda}(\boldsymbol{\beta})-\phi_{\lambda}\left(\boldsymbol{\beta}^{*}\right) \leq\left(\boldsymbol{\beta}-\boldsymbol{\beta}^{*}\right)^{T}\left(\nabla \widetilde{\mathcal{L}}_{\lambda}(\boldsymbol{\beta})+\lambda \boldsymbol{\xi}\right) \leq \lambda / 2 \cdot\left\|\boldsymbol{\beta}-\boldsymbol{\beta}^{*}\right\|_{1} .
$$


Here the second inequality follows from (D.13), which is a direct consequence of the assumption that $\omega_{\lambda}(\boldsymbol{\beta}) \leq \lambda / 2$. Separating the support of $\boldsymbol{\beta}-\boldsymbol{\beta}^{*}$ into $S^{*}$ and $\overline{S^{*}}$, we obtain

$$
\begin{aligned}
\phi_{\lambda}(\boldsymbol{\beta})-\phi_{\lambda}\left(\boldsymbol{\beta}^{*}\right) & \leq \lambda / 2 \cdot\left\|\boldsymbol{\beta}-\boldsymbol{\beta}^{*}\right\|_{1} \\
& \leq \lambda / 2 \cdot\left\|\left(\boldsymbol{\beta}-\boldsymbol{\beta}^{*}\right)_{S^{*}}\right\|_{1}+\lambda / 2 \cdot\left\|\left(\boldsymbol{\beta}-\boldsymbol{\beta}^{*}\right)_{S^{*}}\right\|_{1} .
\end{aligned}
$$

Now we derive an upper bound of $\left\|\left(\boldsymbol{\beta}-\boldsymbol{\beta}^{*}\right)_{\overline{S^{*}}}\right\|_{1}$ on the right-hand side of (D.35). On the left-hand side of (D.27), we have $\rho_{-}-\zeta_{-}>0$, which gives

$$
\begin{aligned}
& \left(\lambda / 2-\left\|\nabla \mathcal{L}\left(\boldsymbol{\beta}^{*}\right)\right\|_{\infty}\right)\left\|\left(\boldsymbol{\beta}-\boldsymbol{\beta}^{*}\right)_{\overline{S^{*}}}\right\|_{1} \\
& \quad \leq\left(3 \lambda / 2+\left\|\nabla \mathcal{L}\left(\boldsymbol{\beta}^{*}\right)\right\|_{\infty}+\left\|\nabla \mathcal{Q}_{\lambda}\left(\boldsymbol{\beta}^{*}\right)\right\|_{\infty}\right)\left\|\left(\boldsymbol{\beta}-\boldsymbol{\beta}^{*}\right)_{S^{*}}\right\|_{1} .
\end{aligned}
$$

Note that in (D.36) we have $\left\|\nabla \mathcal{L}\left(\boldsymbol{\beta}^{*}\right)\right\|_{\infty} \leq \lambda / 8$ by (D.28), and $\left\|\nabla \mathcal{Q}_{\lambda}\left(\boldsymbol{\beta}^{*}\right)\right\|_{\infty} \leq$ $\lambda$ by (D.29). Hence we have

$$
(\lambda / 2-\lambda / 8)\left\|\left(\boldsymbol{\beta}-\boldsymbol{\beta}^{*}\right)_{S^{*}}\right\|_{1} \leq(3 \lambda / 2+\lambda / 8+\lambda)\left\|\left(\boldsymbol{\beta}-\boldsymbol{\beta}^{*}\right)_{S^{*}}\right\|_{1},
$$

which implies $\left\|\left(\boldsymbol{\beta}-\boldsymbol{\beta}^{*}\right)_{\overline{S^{*}}}\right\|_{1} \leq 7\left\|\left(\boldsymbol{\beta}-\boldsymbol{\beta}^{*}\right)_{S^{*}}\right\|_{1}$. Plugging this into the righthand side of (D.35), we obtain

$$
\begin{aligned}
\phi_{\lambda}(\boldsymbol{\beta})-\phi_{\lambda}\left(\boldsymbol{\beta}^{*}\right) & \leq(\lambda / 2+7 \lambda / 2)\left\|\left(\boldsymbol{\beta}-\boldsymbol{\beta}^{*}\right)_{S^{*}}\right\|_{1} \\
& \leq 4 \lambda \sqrt{s^{*}}\left\|\left(\boldsymbol{\beta}-\boldsymbol{\beta}^{*}\right)_{S^{*}}\right\|_{2} \leq 4 \lambda \sqrt{s^{*}}\left\|\boldsymbol{\beta}-\boldsymbol{\beta}^{*}\right\|_{2} .
\end{aligned}
$$

Plugging the upper bound of $\left\|\boldsymbol{\beta}-\boldsymbol{\beta}^{*}\right\|_{2}$ in (D.31) into the right-hand side of (D.38), we obtain

$$
\phi_{\lambda}(\boldsymbol{\beta})-\phi_{\lambda}\left(\boldsymbol{\beta}^{*}\right) \leq \frac{21 / 2}{\rho_{-}-\zeta_{-}} \lambda^{2} s^{*}
$$

Hence we reach the second conclusion.

\section{D.4. Proof of Lemma 5.3 in Wang et al. (2014a).}

Proof. Since $\left\|\boldsymbol{\beta}_{\overline{S^{*}}}\right\|_{0} \leq \widetilde{s}$ and $\left\|\boldsymbol{\beta}_{\overline{S^{*}}}^{*}\right\|_{0}=0$, we have $\left\|\left(\boldsymbol{\beta}-\boldsymbol{\beta}^{*}\right)_{S^{*}}\right\|_{0} \leq \widetilde{s}$. For logistic loss, we further have $\|\boldsymbol{\beta}\|_{2} \leq R$ and $\left\|\boldsymbol{\beta}^{*}\right\|_{2} \leq R$, where $R$ is specified in Definition 4.3 of Wang et al. (2014a). Therefore, Lemma 5.1 of Wang et al. (2014a) gives

$$
\widetilde{\mathcal{L}}_{\lambda}\left(\boldsymbol{\beta}^{*}\right)+\left(\boldsymbol{\beta}-\boldsymbol{\beta}^{*}\right)^{T} \nabla \widetilde{\mathcal{L}}_{\lambda}\left(\boldsymbol{\beta}^{*}\right)+\frac{\rho_{-}-\zeta_{-}}{2}\left\|\boldsymbol{\beta}^{*}-\boldsymbol{\beta}\right\|_{2}^{2} \leq \widetilde{\mathcal{L}}_{\lambda}(\boldsymbol{\beta}) .
$$

Recall that $\phi_{\lambda}(\boldsymbol{\beta})=\widetilde{\mathcal{L}}_{\lambda}(\boldsymbol{\beta})+\lambda\|\boldsymbol{\beta}\|_{1}$. Hence, from our assumption that

$$
\phi_{\lambda}(\boldsymbol{\beta})-\phi_{\lambda}\left(\boldsymbol{\beta}^{*}\right) \leq \frac{21 / 2}{\rho_{-}-\zeta_{-}} \lambda^{2} s^{*}
$$


we obtain

$$
\widetilde{\mathcal{L}}_{\lambda}(\boldsymbol{\beta})-\widetilde{\mathcal{L}}_{\lambda}\left(\boldsymbol{\beta}^{*}\right)+\lambda\left(\|\boldsymbol{\beta}\|_{1}-\left\|\boldsymbol{\beta}^{*}\right\|_{1}\right) \leq \frac{21 / 2}{\rho_{-}-\zeta_{-}} \lambda^{2} s^{*}
$$

Plugging (D.39) into the left-hand side of (D.40), we have

$$
\left(\boldsymbol{\beta}-\boldsymbol{\beta}^{*}\right)^{T} \nabla \widetilde{\mathcal{L}}_{\lambda}\left(\boldsymbol{\beta}^{*}\right)+\frac{\rho_{-}-\zeta_{-}}{2}\left\|\boldsymbol{\beta}^{*}-\boldsymbol{\beta}\right\|_{2}^{2}+\lambda\left(\|\boldsymbol{\beta}\|_{1}-\left\|\boldsymbol{\beta}^{*}\right\|_{1}\right) \leq \frac{21 / 2}{\rho_{-}-\zeta_{-}} \lambda^{2} s^{*} .
$$

Moving $\left(\boldsymbol{\beta}-\boldsymbol{\beta}^{*}\right)^{T} \nabla \widetilde{\mathcal{L}}_{\lambda}\left(\boldsymbol{\beta}^{*}\right)+\lambda\left(\|\boldsymbol{\beta}\|_{1}-\left\|\boldsymbol{\beta}^{*}\right\|_{1}\right)$ to its right-hand side yields

$$
\begin{aligned}
& \frac{\rho_{-}-\zeta_{-}}{2}\left\|\boldsymbol{\beta}^{*}-\boldsymbol{\beta}\right\|_{2}^{2} \\
& \leq \frac{21 / 2}{\rho_{-}-\zeta_{-}} \lambda^{2} s^{*} \underbrace{-\left(\boldsymbol{\beta}-\boldsymbol{\beta}^{*}\right)^{T} \nabla \widetilde{\mathcal{L}_{\lambda}}\left(\boldsymbol{\beta}^{*}\right)}_{(\mathrm{i})}+\lambda \underbrace{\left(\left\|\boldsymbol{\beta}^{*}\right\|_{1}-\|\boldsymbol{\beta}\|_{1}\right)}_{(\cdot i)} .
\end{aligned}
$$

For term (i) in (D.41), following the same way we obtain the lower bound of term (i) in (D.14) (in the proof of Lemma 5.2), we can obtain the same result as in (D.21), which implies

$$
\begin{gathered}
-\left(\boldsymbol{\beta}-\boldsymbol{\beta}^{*}\right)^{T} \nabla \widetilde{\mathcal{L}}_{\lambda}\left(\boldsymbol{\beta}^{*}\right) \leq\left\|\left(\boldsymbol{\beta}-\boldsymbol{\beta}^{*}\right)_{S^{*}}\right\|_{1}\left\|\nabla \mathcal{L}\left(\boldsymbol{\beta}^{*}\right)\right\|_{\infty}+\left\|\left(\boldsymbol{\beta}-\boldsymbol{\beta}^{*}\right)_{S^{*}}\right\|_{1}\left\|\nabla \mathcal{L}\left(\boldsymbol{\beta}^{*}\right)\right\|_{\infty} \\
(\mathrm{D} .42) \\
+\left\|\left(\boldsymbol{\beta}-\boldsymbol{\beta}^{*}\right)_{S^{*}}\right\|_{1}\left\|\nabla \mathcal{Q}_{\lambda}\left(\boldsymbol{\beta}^{*}\right)\right\|_{\infty} .
\end{gathered}
$$

For term (ii) in (D.41), separating the support of $\boldsymbol{\beta}$ and $\boldsymbol{\beta}^{*}$ into $S^{*}$ and $\overline{S^{*}}$ respectively, we obtain

$$
\left\|\boldsymbol{\beta}^{*}\right\|_{1}-\|\boldsymbol{\beta}\|_{1}=\left\|\boldsymbol{\beta}_{S^{*}}^{*}\right\|_{1}+\left\|\boldsymbol{\beta}_{\overline{S^{*}}}^{*}\right\|_{1}-\left(\left\|\boldsymbol{\beta}_{S^{*}}\right\|_{1}+\left\|\boldsymbol{\beta}_{\overline{S^{*}}}\right\|_{1}\right) .
$$

Note that $\boldsymbol{\beta}_{\overline{S^{*}}}^{*}=\mathbf{0}$, which gives $\boldsymbol{\beta}_{\overline{S^{*}}}=\boldsymbol{\beta}_{\overline{S^{*}}}-\boldsymbol{\beta}_{\overline{S^{*}}}^{*}=\left(\boldsymbol{\beta}-\boldsymbol{\beta}^{*}\right)_{\overline{S^{*}}}$. Hence, from (D.43) we have

$$
\begin{aligned}
\left\|\boldsymbol{\beta}^{*}\right\|_{1}-\|\boldsymbol{\beta}\|_{1} & =\left\|\boldsymbol{\beta}_{S^{*}}^{*}\right\|_{1}-\left\|\boldsymbol{\beta}_{S^{*}}\right\|_{1}-\left\|\left(\boldsymbol{\beta}-\boldsymbol{\beta}^{*}\right)_{\overline{S^{*}}}\right\|_{1} \\
& \leq\left\|\left(\boldsymbol{\beta}-\boldsymbol{\beta}^{*}\right)_{S^{*}}\right\|_{1}-\left\|\left(\boldsymbol{\beta}-\boldsymbol{\beta}^{*}\right)_{\overline{S^{*}}}\right\|_{1},
\end{aligned}
$$

where the inequality follows from the triangle inequality. Plugging (D.42) and (D.44) into the right-hand side of (D.41), we obtain

$$
\begin{aligned}
& \frac{\rho_{-}-\zeta_{-}}{2}\left\|\boldsymbol{\beta}^{*}-\boldsymbol{\beta}\right\|_{2}^{2}
\end{aligned}
$$

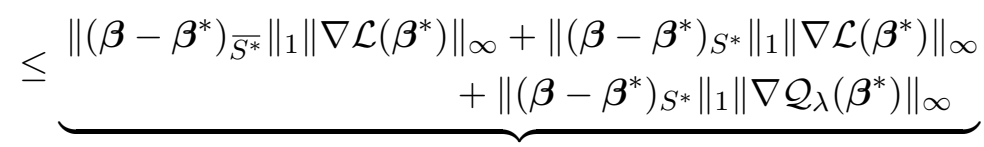

$$
\begin{aligned}
& +\lambda \underbrace{\left(\left\|\left(\boldsymbol{\beta}-\boldsymbol{\beta}^{*}\right)_{S^{*}}\right\|_{1}-\left\|\left(\boldsymbol{\beta}-\boldsymbol{\beta}^{*}\right)_{\overline{S^{*}}}\right\|_{1}\right)}+\frac{21 / 2}{\rho_{-}-\zeta_{-}} \lambda^{2} s^{*} .
\end{aligned}
$$

(ii) in (D.41) 
Rearranging the terms in (D.45), we obtain

$$
\begin{aligned}
& \frac{\rho_{-}-\zeta_{-}}{2}\left\|\boldsymbol{\beta}-\boldsymbol{\beta}^{*}\right\|_{2}^{2}+\underbrace{\left(\lambda-\left\|\nabla \mathcal{L}\left(\boldsymbol{\beta}^{*}\right)\right\|_{\infty}\right)\left\|\left(\boldsymbol{\beta}-\boldsymbol{\beta}^{*}\right)_{\overline{S^{*}}}\right\|_{1}}_{(\mathrm{i})} \\
& \leq(\lambda+\underbrace{\left\|\nabla \mathcal{L}\left(\boldsymbol{\beta}^{*}\right)\right\|_{\infty}}_{(\mathrm{ii})}+\underbrace{\left\|\nabla \mathcal{Q}_{\lambda}\left(\boldsymbol{\beta}^{*}\right)\right\|_{\infty}}_{(\mathrm{iii})})\left\|\left(\boldsymbol{\beta}-\boldsymbol{\beta}^{*}\right)_{S^{*}}\right\|_{1}+\frac{21 / 2}{\rho_{-}-\zeta_{-}} \lambda^{2} s^{*} .
\end{aligned}
$$

By (4.1) in Assumption 4.1 of Wang et al. (2014a) and $\lambda \geq \lambda_{\text {tgt }}$, for term (ii) in (D.46), we have

$$
\left\|\nabla \mathcal{L}\left(\boldsymbol{\beta}^{*}\right)\right\|_{\infty} \leq \lambda_{\text {tgt }} / 8 \leq \lambda / 8 .
$$

Moreover, (D.47) implies that term (i) in (D.46) is positive. For term (iii) in (D.46), since $\mathcal{Q}_{\lambda}(\boldsymbol{\beta})=\sum_{j=1}^{d} q_{\lambda}\left(\beta_{j}\right)$, where $q_{\lambda}\left(\beta_{j}\right)$ satisfies regularity condition (d), we have

$$
\left\|\nabla \mathcal{Q}_{\lambda}\left(\boldsymbol{\beta}^{*}\right)\right\|_{\infty} \leq \max _{1 \leq j \leq d}\left|q_{\lambda}^{\prime}\left(\beta_{j}^{*}\right)\right| \leq \lambda .
$$

Therefore, from (D.48) we obtain

$$
\begin{aligned}
& \frac{\rho_{-}-\zeta_{-}}{2}\left\|\boldsymbol{\beta}-\boldsymbol{\beta}^{*}\right\|_{2}^{2} \\
& \leq\left(\lambda+\left\|\nabla \mathcal{L}\left(\boldsymbol{\beta}^{*}\right)\right\|_{\infty}+\left\|\nabla \mathcal{Q}_{\lambda}\left(\boldsymbol{\beta}^{*}\right)\right\|_{\infty}\right)\left\|\left(\boldsymbol{\beta}-\boldsymbol{\beta}^{*}\right)_{S^{*}}\right\|_{1}+\frac{21 / 2}{\rho_{-}-\zeta_{-}} \lambda^{2} s^{*} \\
& \leq(\lambda+\lambda / 8+\lambda)\left\|\left(\boldsymbol{\beta}-\boldsymbol{\beta}^{*}\right)_{S^{*}}\right\|_{1}+\frac{21 / 2}{\rho_{-}-\zeta_{-}} \lambda^{2} s^{*} \\
& \leq 17 / 8 \cdot \lambda\left\|\left(\boldsymbol{\beta}-\boldsymbol{\beta}^{*}\right)_{S^{*}}\right\|_{1}+\frac{21 / 2}{\rho_{-}-\zeta_{-}} \lambda^{2} s^{*}
\end{aligned}
$$

To further obtain an upper bound of the right-hand side of (D.49), we discuss two cases regarding the relationship between $\left\|\left(\boldsymbol{\beta}-\boldsymbol{\beta}^{*}\right)_{S^{*}}\right\|_{1}$ and $\lambda s^{*}$.

- If $7 /\left(\rho_{-}-\zeta_{-}\right) \cdot \lambda s^{*}<\left\|\left(\boldsymbol{\beta}-\boldsymbol{\beta}^{*}\right)_{S^{*}}\right\|_{1}$, then we have

$$
\frac{21 / 2}{\rho_{-}-\zeta_{-}} \lambda^{2} s^{*}<3 / 2 \cdot \lambda\left\|\left(\boldsymbol{\beta}-\boldsymbol{\beta}^{*}\right)_{S^{*}}\right\|_{1} .
$$

Plugging this into the right-hand side of (D.49), we obtain

$$
\begin{aligned}
\frac{\rho_{-}-\zeta_{-}}{2}\left\|\boldsymbol{\beta}-\boldsymbol{\beta}^{*}\right\|_{2}^{2} & \leq(17 / 8 \cdot \lambda+3 / 2 \cdot \lambda)\left\|\left(\boldsymbol{\beta}-\boldsymbol{\beta}^{*}\right)_{S^{*}}\right\|_{1} \\
& \leq 29 / 8 \cdot \lambda \sqrt{s^{*}}\left\|\left(\boldsymbol{\beta}-\boldsymbol{\beta}^{*}\right)_{S^{*}}\right\|_{2} \\
& \leq 29 / 8 \cdot \lambda \sqrt{s^{*}}\left\|\boldsymbol{\beta}-\boldsymbol{\beta}^{*}\right\|_{2} .
\end{aligned}
$$


Dividing $\left\|\boldsymbol{\beta}^{*}-\boldsymbol{\beta}\right\|_{2}$ on both sides, we have

$$
\left\|\boldsymbol{\beta}-\boldsymbol{\beta}^{*}\right\|_{2} \leq \frac{29 / 4}{\rho_{-}-\zeta_{-}} \lambda \sqrt{s^{*}}
$$

- If $\left\|\left(\boldsymbol{\beta}-\boldsymbol{\beta}^{*}\right)_{S^{*}}\right\|_{1} \leq 7 /\left(\rho_{-}-\zeta_{-}\right) \cdot \lambda s^{*}$, then we have

$$
17 / 8 \cdot \lambda\left\|\left(\boldsymbol{\beta}-\boldsymbol{\beta}^{*}\right)_{S^{*}}\right\|_{1}<\frac{119 / 8}{\rho_{-}-\zeta_{-}} \lambda^{2} s^{*}
$$

Plugging this into the right-hand side of (D.49), we obtain

$$
\begin{aligned}
\frac{\rho_{-}-\zeta_{-}}{2}\left\|\boldsymbol{\beta}-\boldsymbol{\beta}^{*}\right\|_{2}^{2} & \leq \frac{119 / 8}{\rho_{-}-\zeta_{-}} \lambda^{2} s^{*}+\frac{21 / 2}{\rho_{-}-\zeta_{-}} \lambda^{2} s^{*} \\
& =\frac{203 / 8}{\rho_{-}-\zeta_{-}} \lambda^{2} s^{*}
\end{aligned}
$$

which implies

$$
\left\|\boldsymbol{\beta}-\boldsymbol{\beta}^{*}\right\|_{2} \leq \frac{\sqrt{203} / 2}{\rho_{-}-\zeta_{-}} \lambda \sqrt{s^{*}} .
$$

Combining (D.50) and (D.52), since $\max \{29 / 4, \sqrt{203} / 2\} \leq 15 / 2$, we obtain

$$
\left\|\boldsymbol{\beta}-\boldsymbol{\beta}^{*}\right\|_{2}<\frac{15 / 2}{\rho_{-}-\zeta_{-}} \lambda \sqrt{s^{*}}
$$

Hence we conclude the proof.

\section{D.5. Proof of Lemma 5.4 in Wang et al. (2014a).}

Proof. Recall the proximal-gradient update step defined in (3.8) of Wang et al. (2014a) with $\Omega=\mathbb{R}^{d}$, i.e., $R=+\infty$, takes the form

$$
\left(\mathcal{T}_{L, \lambda}(\boldsymbol{\beta} ;+\infty)\right)_{j}= \begin{cases}0 & \text { if }\left|\bar{\beta}_{j}\right| \leq \lambda / L \\ \operatorname{sign}\left(\bar{\beta}_{j}\right)\left(\left|\bar{\beta}_{j}\right|-\lambda / L\right) & \text { if }\left|\bar{\beta}_{j}\right|>\lambda / L\end{cases}
$$

for $j=1, \ldots, d$, where

$$
\overline{\boldsymbol{\beta}}=\boldsymbol{\beta}-\frac{1}{L} \nabla \widetilde{\mathcal{L}}_{\lambda}(\boldsymbol{\beta}),
$$

and $\bar{\beta}_{j}$ is the $j$-th dimension of $\overline{\boldsymbol{\beta}}$. Furthermore, if $\Omega=B_{2}(R)$ of radius $R \in(0, \infty), \mathcal{T}_{L, \lambda}(\boldsymbol{\beta} ; R)$ can be obtained by projecting $\mathcal{T}_{L, \lambda}(\boldsymbol{\beta} ;+\infty)$ shown in (D.53) onto $B_{2}(R)$, i.e.,

$$
\mathcal{T}_{L, \lambda}(\boldsymbol{\beta} ; R)= \begin{cases}\mathcal{T}_{L, \lambda}(\boldsymbol{\beta} ;+\infty) & \text { if }\left\|\mathcal{T}_{L, \lambda}(\boldsymbol{\beta} ;+\infty)\right\|_{2}<R \\ \frac{R \cdot \mathcal{T}_{L, \lambda}(\boldsymbol{\beta} ;+\infty)}{\left\|\mathcal{T}_{L, \lambda}(\boldsymbol{\beta} ;+\infty)\right\|_{2}} & \text { if }\left\|\mathcal{T}_{L, \lambda}(\boldsymbol{\beta} ;+\infty)\right\|_{2} \geq R\end{cases}
$$

Note that $\mathcal{T}_{L, \lambda}(\boldsymbol{\beta} ;+\infty)$ and $\mathcal{T}_{L, \lambda}(\boldsymbol{\beta} ; R)$ have exactly the same sparsity pattern. 
Hence we focus on analyzing the sparsity pattern of $\mathcal{T}_{L, \lambda}(\boldsymbol{\beta} ;+\infty)$ in the following.

In fact, update scheme (D.53) defines a soft-thresholding operation on $\overline{\boldsymbol{\beta}}$ defined in (D.54), with the threshold value $\lambda / L$. To show $\left\|\left(\mathcal{T}_{L, \lambda}(\boldsymbol{\beta} ;+\infty)\right)_{\overline{S^{*}}}\right\|_{0} \leq$ $\widetilde{s}$, we need to prove that, for $j \in \overline{S^{*}}$, the number of $j$ 's such that $\left|\bar{\beta}_{j}\right|>\lambda / L$ is no more than $\widetilde{s}$. To achieve this goal, we first reformulate $\overline{\boldsymbol{\beta}}$ as

$$
\overline{\boldsymbol{\beta}}=\boldsymbol{\beta}-\frac{1}{L} \nabla \widetilde{\mathcal{L}}_{\lambda}(\boldsymbol{\beta})=\boldsymbol{\beta}-\frac{1}{L} \nabla \widetilde{\mathcal{L}}_{\lambda}\left(\boldsymbol{\beta}^{*}\right)+\frac{1}{L}\left(\nabla \widetilde{\mathcal{L}}_{\lambda}\left(\boldsymbol{\beta}^{*}\right)-\nabla \widetilde{\mathcal{L}}_{\lambda}(\boldsymbol{\beta})\right)
$$

Then it suffices to prove there exist integers $\widetilde{s}_{1}, \widetilde{s}_{2}$ and $\widetilde{s}_{3}$, which satisfy $\widetilde{s}_{1}+\widetilde{s}_{2}+\widetilde{s}_{3} \leq \widetilde{s}$, such that

$$
\begin{array}{r}
\left|\left\{j \in \overline{S^{*}}:\left|\beta_{j}\right| \geq 1 / 4 \cdot \lambda / L\right\}\right| \leq \widetilde{s}_{1}, \\
\left|\left\{j \in \overline{S^{*}}:\left|\left(\nabla \widetilde{\mathcal{L}}_{\lambda}\left(\boldsymbol{\beta}^{*}\right) / L\right)_{j}\right|>1 / 8 \cdot \lambda / L\right\}\right| \leq \widetilde{s}_{2}, \\
\left|\left\{j \in \overline{S^{*}}:\left|\left(\nabla \widetilde{\mathcal{L}}_{\lambda}(\boldsymbol{\beta}) / L-\nabla \widetilde{\mathcal{L}}_{\lambda}\left(\boldsymbol{\beta}^{*}\right) / L\right)_{j}\right| \geq 5 / 8 \cdot \lambda / L\right\}\right| \leq \widetilde{s}_{3} .
\end{array}
$$

This is because, if (D.57)-(D.59) hold, then there are at most $\widetilde{s}_{1}+\widetilde{s}_{2}+\widetilde{s}_{3} \leq \widetilde{s}$ coordinates $j \in \overline{S^{*}}$ such that

$$
\left|\beta_{j}\right|+\left|\left(\nabla \widetilde{\mathcal{L}}_{\lambda}\left(\boldsymbol{\beta}^{*}\right) / L\right)_{j}\right|+\left|\left(\nabla \widetilde{\mathcal{L}}_{\lambda}(\boldsymbol{\beta}) / L-\nabla \widetilde{\mathcal{L}}_{\lambda}\left(\boldsymbol{\beta}^{*}\right) / L\right)_{j}\right|>\lambda / L .
$$

Since by the triangular inequality (D.56) implies

$$
\left|\bar{\beta}_{j}\right| \leq\left|\beta_{j}\right|+\left|\left(\nabla \widetilde{\mathcal{L}}_{\lambda}\left(\boldsymbol{\beta}^{*}\right) / L\right)_{j}\right|+\left|\left(\nabla \widetilde{\mathcal{L}}_{\lambda}(\boldsymbol{\beta}) / L-\nabla \widetilde{\mathcal{L}}_{\lambda}\left(\boldsymbol{\beta}^{*}\right) / L\right)_{j}\right|,
$$

the number of coordinates $j \in \overline{S^{*}}$ such that $\left|\bar{\beta}_{j}\right|>\lambda / L$ is also upper bounded by $\widetilde{s}_{1}+\widetilde{s}_{2}+\widetilde{s}_{3} \leq \widetilde{s}$. In the following, we will prove (D.58)-(D.59) and specify the corresponding $\widetilde{s}_{1}, \widetilde{s}_{2}$ and $\widetilde{s}_{3}$.

Proof of (D.57): Note that for $j \in \overline{S^{*}}$, we have $\beta_{j}^{*}=0$. Hence we have

$$
\left|\left\{j \in \overline{S^{*}}:\left|\beta_{j}\right| \geq 1 / 4 \cdot \lambda / L\right\}\right|=\left|\left\{j \in \overline{S^{*}}:\left|\beta_{j}-\beta_{j}^{*}\right| \geq 1 / 4 \cdot \lambda / L\right\}\right| .
$$

Meanwhile, note that

$$
\begin{aligned}
& \frac{\lambda}{4 L}\left|\left\{j \in \overline{S^{*}}:\left|\beta_{j}-\beta_{j}^{*}\right| \geq 1 / 4 \cdot \lambda / L\right\}\right| \\
& \leq \sum_{j \in \overline{S^{*}}}\left|\beta_{j}-\beta_{j}^{*}\right| \cdot \mathbb{1}\left(\left|\beta_{j}-\beta_{j}^{*}\right| \geq 1 / 4 \cdot \lambda / L\right) \\
& \leq \sum_{j \in \overline{S^{*}}}\left|\beta_{j}-\beta_{j}^{*}\right| \\
& =\left\|\left(\boldsymbol{\beta}-\boldsymbol{\beta}^{*}\right)_{\overline{S^{*}}}\right\|_{1} .
\end{aligned}
$$


Plugging (D.61) into the right-hand side of (D.60), we obtain

$$
\left|\left\{j \in \overline{S^{*}}:\left|\beta_{j}\right| \geq 1 / 4 \cdot \lambda / L\right\}\right| \leq \frac{4 L}{\lambda}\left\|\left(\boldsymbol{\beta}-\boldsymbol{\beta}^{*}\right)_{\overline{S^{*}}}\right\|_{1} .
$$

Now we provide an upper bound of $\left\|\left(\boldsymbol{\beta}-\boldsymbol{\beta}^{*}\right)_{\bar{S}^{*}}\right\|_{1}$. Following the same way we derive (D.46) in the proof of Lemma 5.3 , we can obtain

$$
\begin{aligned}
& \frac{\rho_{-}-\zeta_{-}}{2}\left\|\boldsymbol{\beta}-\boldsymbol{\beta}^{*}\right\|_{2}^{2}+\left(\lambda-\left\|\nabla \mathcal{L}\left(\boldsymbol{\beta}^{*}\right)\right\|_{\infty}\right)\left\|\left(\boldsymbol{\beta}-\boldsymbol{\beta}^{*}\right)_{\overline{S^{*}}}\right\|_{1} \\
& \leq\left(\lambda+\left\|\nabla \mathcal{L}\left(\boldsymbol{\beta}^{*}\right)\right\|_{\infty}+\left\|\nabla \mathcal{Q}_{\lambda}\left(\boldsymbol{\beta}^{*}\right)\right\|_{\infty}\right)\left\|\left(\boldsymbol{\beta}-\boldsymbol{\beta}^{*}\right)_{S^{*}}\right\|_{1}+\frac{21 / 2}{\rho_{-}-\zeta_{-}} \lambda^{2} s^{*} .
\end{aligned}
$$

According to (4.3) in Wang et al. (2014a), we have $\rho_{-}-\zeta_{-}>0$. Hence (D.63) implies

$$
\begin{aligned}
& \left(\lambda-\left\|\nabla \mathcal{L}\left(\boldsymbol{\beta}^{*}\right)\right\|_{\infty}\right)\left\|\left(\boldsymbol{\beta}-\boldsymbol{\beta}^{*}\right)_{S^{*}}\right\|_{1} \\
& \leq\left(\lambda+\left\|\nabla \mathcal{L}\left(\boldsymbol{\beta}^{*}\right)\right\|_{\infty}+\left\|\nabla \mathcal{Q}_{\lambda}\left(\boldsymbol{\beta}^{*}\right)\right\|_{\infty}\right)\left\|\left(\boldsymbol{\beta}-\boldsymbol{\beta}^{*}\right)_{S^{*}}\right\|_{1}+\frac{21 / 2}{\rho_{-}-\zeta_{-}} \lambda^{2} s^{*} .
\end{aligned}
$$

By (4.1) in Assumption 4.1 of Wang et al. (2014a) and $\lambda \geq \lambda_{\text {tgt }}$, we have

$$
\left\|\nabla \mathcal{L}\left(\boldsymbol{\beta}^{*}\right)\right\|_{\infty} \leq \lambda_{\text {tgt }} / 8 \leq \lambda / 8 .
$$

Meanwhile, since $\mathcal{Q}_{\lambda}(\boldsymbol{\beta})=\sum_{j=1}^{d} q_{\lambda}\left(\beta_{j}\right)$ and $q_{\lambda}\left(\beta_{j}\right)$ satisfies regularity condition (d) in Wang et al. (2014a), we have

$$
\left\|\nabla \mathcal{Q}_{\lambda}\left(\boldsymbol{\beta}^{*}\right)\right\|_{\infty}=\max _{1 \leq j \leq d}\left|q_{\lambda}^{\prime}\left(\beta_{j}^{*}\right)\right| \leq \lambda .
$$

Plugging (D.65) and (D.66) into (D.64) and dividing $\lambda$ on both sides, we obtain

$$
7 / 8 \cdot\left\|\left(\boldsymbol{\beta}-\boldsymbol{\beta}^{*}\right)_{S^{*}}\right\|_{1} \leq 17 / 8 \cdot\left\|\left(\boldsymbol{\beta}-\boldsymbol{\beta}^{*}\right)_{S^{*}}\right\|_{1}+\frac{21 / 2}{\rho_{-}-\zeta_{-}} \lambda s^{*} .
$$

Now we discuss two cases regarding the relationship between $\left\|\left(\boldsymbol{\beta}-\boldsymbol{\beta}^{*}\right)_{S^{*}}\right\|_{1}$ and $\lambda s^{*}$.

- If $7 /\left(\rho_{-}-\zeta_{-}\right) \cdot \lambda s^{*}<\left\|\left(\boldsymbol{\beta}-\boldsymbol{\beta}^{*}\right)_{S^{*}}\right\|_{1}$, then we have

$$
\frac{21 / 2}{\rho_{-}-\zeta_{-}} \lambda s^{*} \leq 3 / 2 \cdot\left\|\left(\boldsymbol{\beta}-\boldsymbol{\beta}^{*}\right)_{S^{*}}\right\|_{1} \cdot
$$

Plugging this into the right-hand side of (D.67), we obtain

$$
\left\|\left(\boldsymbol{\beta}-\boldsymbol{\beta}^{*}\right)_{S^{*}}\right\|_{1} \leq 29 / 7 \cdot\left\|\left(\boldsymbol{\beta}-\boldsymbol{\beta}^{*}\right)_{S^{*}}\right\|_{1},
$$

which implies

$$
\begin{aligned}
\left\|\left(\boldsymbol{\beta}-\boldsymbol{\beta}^{*}\right)_{\overline{S^{*}}}\right\|_{1} \leq 29 / 7 \cdot\left\|\left(\boldsymbol{\beta}-\boldsymbol{\beta}^{*}\right)_{S^{*}}\right\|_{1} & \leq 29 / 7 \cdot \sqrt{s^{*}}\left\|\left(\boldsymbol{\beta}-\boldsymbol{\beta}^{*}\right)_{S^{*}}\right\|_{2} \\
& \leq 29 / 7 \cdot \sqrt{s^{*}}\left\|\boldsymbol{\beta}-\boldsymbol{\beta}^{*}\right\|_{2} .
\end{aligned}
$$


Plugging the upper bound of $\left\|\boldsymbol{\beta}-\boldsymbol{\beta}^{*}\right\|_{2}$ in Lemma 5.3 of Wang et al. (2014a) into the right-hand side of (D.68), we obtain

$$
\left\|\left(\boldsymbol{\beta}-\boldsymbol{\beta}^{*}\right)_{\overline{S^{*}}}\right\|_{1} \leq 29 / 7 \cdot \sqrt{s^{*}} \cdot \frac{15 / 2}{\rho_{-}-\zeta_{-}} \lambda \sqrt{s^{*}}=\frac{435 / 14}{\rho_{-}-\zeta_{-}} \lambda s^{*} .
$$

- If $\left\|\left(\boldsymbol{\beta}-\boldsymbol{\beta}^{*}\right)_{S^{*}}\right\|_{1} \leq 7 /\left(\rho_{-}-\zeta_{-}\right) \cdot \lambda s^{*}$, then plugging this into the right-hand side of (D.67), we obtain

$$
\left\|\left(\boldsymbol{\beta}-\boldsymbol{\beta}^{*}\right)_{\overline{S^{*}}}\right\|_{1} \leq 8 / 7 \cdot \frac{17 / 8 \cdot 7+21 / 2}{\rho_{-}-\zeta_{-}} \lambda s^{*}=\frac{29}{\rho_{-}-\zeta_{-}} \lambda s^{*} .
$$

Combining (D.69) and (D.70), we obtain

$$
\left\|\left(\boldsymbol{\beta}-\boldsymbol{\beta}^{*}\right)_{\overline{S^{*}}}\right\|_{1} \leq \frac{\max \{435 / 14,29\}}{\rho_{-}-\zeta_{-}} \lambda s^{*} \leq \frac{435 / 14}{\rho_{-}-\zeta_{-}} \lambda s^{*} .
$$

Plugging this into the right-hand side of (D.62), we obtain

$$
\left|\left\{j \in \overline{S^{*}}:\left|\beta_{j}\right| \geq 1 / 4 \cdot \lambda / L\right\}\right| \leq \frac{4 L}{\lambda} \cdot \frac{435 / 14}{\rho_{-}-\zeta_{-}} \lambda s^{*}<\frac{125 L}{\rho_{-}-\zeta_{-}} s^{*} .
$$

Meanwhile, since we assume $L<2\left(\rho_{+}-\zeta_{+}\right)$, we have

$$
\left|\left\{j \in \overline{S^{*}}:\left|\beta_{j}\right| \geq 1 / 4 \cdot \lambda / L\right\}\right|<250 \cdot \frac{\rho_{+}-\zeta_{+}}{\rho_{-}-\zeta_{-}} \cdot s^{*}=250 \kappa s^{*}
$$

where the last equality follows from the definition of the condition number $\kappa$ in (4.5). Therefore we obtain (D.57) by setting $\widetilde{s}_{1}=250 \kappa s^{*}$.

Proof of (D.58): Recall that $\nabla \widetilde{\mathcal{L}}_{\lambda}(\boldsymbol{\beta})=\mathcal{L}(\boldsymbol{\beta})+\mathcal{Q}_{\lambda}(\boldsymbol{\beta})$. Hence we have

$$
\left\|\left(\nabla \widetilde{\mathcal{L}}_{\lambda}\left(\boldsymbol{\beta}^{*}\right)\right)_{\overline{S^{*}}}\right\|_{\infty} \leq\left\|\left(\nabla \mathcal{L}\left(\boldsymbol{\beta}^{*}\right)\right)_{\overline{S^{*}}}\right\|_{\infty}+\left\|\left(\nabla \mathcal{Q}_{\lambda}\left(\boldsymbol{\beta}^{*}\right)\right)_{\overline{S^{*}}}\right\|_{\infty} .
$$

By (4.1) in Assumption 4.1 of Wang et al. (2014a), we have

$$
\left\|\left(\nabla \mathcal{L}\left(\boldsymbol{\beta}^{*}\right)\right)_{\overline{S^{*}}}\right\|_{\infty} \leq\left\|\nabla \mathcal{L}\left(\boldsymbol{\beta}^{*}\right)\right\|_{\infty} \leq \lambda / 8
$$

Recall $\mathcal{Q}_{\lambda}(\boldsymbol{\beta})=\sum_{j=1}^{d} q_{\lambda}\left(\beta_{j}\right)$, where $q_{\lambda}\left(\beta_{j}\right)$ satisfies regularity condition (c) that $q_{\lambda}^{\prime}(0)=0$. Hence we have

$$
\left\|\left(\nabla \mathcal{Q}_{\lambda}\left(\boldsymbol{\beta}^{*}\right)\right)_{\overline{S^{*}}}\right\|_{\infty}=\max _{j \in \overline{S^{*}}}\left|q_{\lambda}^{\prime}\left(\beta_{j}^{*}\right)\right|=\max _{j \in \overline{S^{*}}}\left|q_{\lambda}^{\prime}(0)\right|=0
$$

where the second equation follows from the fact that $\beta_{j}^{*}=0$ for $j \in \overline{S^{*}}$. Plugging (D.73) and (D.72) into the right-hand side of (D.71), we obtain $\left\|\left(\nabla \widetilde{\mathcal{L}}_{\lambda}\left(\boldsymbol{\beta}^{*}\right)\right)_{\overline{S^{*}}}\right\|_{\infty}=\max _{j \in \overline{S^{*}}}\left|\left(\nabla \widetilde{\mathcal{L}}_{\lambda}\left(\boldsymbol{\beta}^{*}\right) / L\right)_{j}\right| \leq \lambda / 8$. Hence we have

$$
\left|\left\{j \in \overline{S^{*}}:\left|\left(\nabla \widetilde{\mathcal{L}}_{\lambda}\left(\boldsymbol{\beta}^{*}\right) / L\right)_{j}\right|>1 / 8 \cdot \lambda / L\right\}\right|=0
$$

Therefore, by setting $\widetilde{s}_{2}=0$, we obtain (D.58). 
Proof of (D.59): Consider an arbitrary subset $S^{\prime}$ such that

$$
S^{\prime} \subseteq\left\{j:\left|\left(\nabla \widetilde{\mathcal{L}_{\lambda}}(\boldsymbol{\beta})-\nabla \widetilde{\mathcal{L}}_{\lambda}\left(\boldsymbol{\beta}^{*}\right)\right)_{j}\right| \geq 5 / 8 \cdot \lambda\right\} .
$$

Let $s^{\prime}=\left|S^{\prime}\right|$. In the sequel we provide an upper bound of $s^{\prime}$. Suppose $\boldsymbol{v} \in \mathbb{R}^{d}$ is chosen such that $v_{j}=\operatorname{sign}\left\{\left(\nabla \widetilde{\mathcal{L}}_{\lambda}(\boldsymbol{\beta})-\nabla \widetilde{\mathcal{L}}_{\lambda}\left(\boldsymbol{\beta}^{*}\right)\right)_{j}\right\}$ for $j \in S^{\prime}$, and $v_{j}=0$ for $j \notin S^{\prime}$. Hence we have

$$
\begin{aligned}
\boldsymbol{v}^{T}\left(\nabla \widetilde{\mathcal{L}}_{\lambda}(\boldsymbol{\beta})-\nabla \widetilde{\mathcal{L}}_{\lambda}\left(\boldsymbol{\beta}^{*}\right)\right) & =\sum_{j \in S^{\prime}} v_{j}\left(\nabla \widetilde{\mathcal{L}}_{\lambda}(\boldsymbol{\beta})-\nabla \widetilde{\mathcal{L}}_{\lambda}\left(\boldsymbol{\beta}^{*}\right)\right)_{j} \\
& =\sum_{j \in S^{\prime}}\left|\left(\nabla \widetilde{\mathcal{L}}_{\lambda}(\boldsymbol{\beta})-\nabla \widetilde{\mathcal{L}}_{\lambda}\left(\boldsymbol{\beta}^{*}\right)\right)_{j}\right| \geq 5 / 8 \cdot \lambda s^{\prime} .
\end{aligned}
$$

Meanwhile, by Cauchy Schwarz inequality we have

$$
\begin{aligned}
\boldsymbol{v}^{T}\left(\nabla \widetilde{\mathcal{L}}_{\lambda}(\boldsymbol{\beta})-\nabla \widetilde{\mathcal{L}}_{\lambda}\left(\boldsymbol{\beta}^{*}\right)\right) & \leq\|\boldsymbol{v}\|_{2}\left\|\nabla \widetilde{\mathcal{L}}_{\lambda}(\boldsymbol{\beta})-\nabla \widetilde{\mathcal{L}}_{\lambda}\left(\boldsymbol{\beta}^{*}\right)\right\|_{2} \\
& \leq \sqrt{s^{\prime}}\left\|\nabla \widetilde{\mathcal{L}}_{\lambda}(\boldsymbol{\beta})-\nabla \widetilde{\mathcal{L}}_{\lambda}\left(\boldsymbol{\beta}^{*}\right)\right\|_{2}
\end{aligned}
$$

where the last inequality follows from the fact that $\|\boldsymbol{v}\|_{2} \leq \sqrt{s^{\prime}}\|\boldsymbol{v}\|_{\infty}=\sqrt{s^{\prime}}$, because $\boldsymbol{v}$ is chosen such that $\|\boldsymbol{v}\|_{0}=s^{\prime}$. Combining (D.75) and (D.76) gives $(\mathrm{D} .77) 5 / 8 \cdot \lambda s^{\prime} \leq \boldsymbol{v}^{T}\left(\nabla \widetilde{\mathcal{L}}_{\lambda}(\boldsymbol{\beta})-\nabla \widetilde{\mathcal{L}}_{\lambda}\left(\boldsymbol{\beta}^{*}\right)\right) \leq \sqrt{s^{\prime}}\left\|\nabla \widetilde{\mathcal{L}}_{\lambda}(\boldsymbol{\beta})-\nabla \widetilde{\mathcal{L}}_{\lambda}\left(\boldsymbol{\beta}^{*}\right)\right\|_{2}$.

Since $\left\|\boldsymbol{\beta}_{\overline{S^{*}}}\right\|_{0} \leq \widetilde{s}$ and $\left\|\boldsymbol{\beta}_{\overline{S^{*}}}^{*}\right\|_{0}=0$, we have $\left\|\left(\boldsymbol{\beta}-\boldsymbol{\beta}^{*}\right)_{\overline{S^{*}}}\right\| \leq \widetilde{s}$. In the setting of logistic loss, we further have $\|\boldsymbol{\beta}\|_{2} \leq R$ and $\left\|\boldsymbol{\beta}^{*}\right\|_{2} \leq R$, where $R$ is specified in Definition 4.3 of Wang et al. (2014a). Therefore, Lemma 5.1 in Wang et al. (2014a) implies that $\widetilde{\mathcal{L}}_{\lambda}(\boldsymbol{\beta})$ is restricted strongly smooth. Hence we have

$$
\widetilde{\mathcal{L}}_{\lambda}(\boldsymbol{\beta}) \leq \widetilde{\mathcal{L}}_{\lambda}\left(\boldsymbol{\beta}^{*}\right)+\left(\boldsymbol{\beta}-\boldsymbol{\beta}^{*}\right)^{T} \nabla \widetilde{\mathcal{L}}_{\lambda}\left(\boldsymbol{\beta}^{*}\right)+\frac{\rho_{+}-\zeta_{+}}{2}\left\|\boldsymbol{\beta}^{*}-\boldsymbol{\beta}\right\|_{2}^{2} .
$$

According to Nesterov (2004, Theorem 2.1.9), the strong smoothness of $\widetilde{\mathcal{L}}_{\lambda}(\boldsymbol{\beta})$ is equivalent to the Lipschitz continuity of its gradient, i.e.,

$$
\left\|\nabla \widetilde{\mathcal{L}}_{\lambda}(\boldsymbol{\beta})-\nabla \widetilde{\mathcal{L}}_{\lambda}\left(\boldsymbol{\beta}^{*}\right)\right\|_{2} \leq\left(\rho_{+}-\zeta_{+}\right)\left\|\boldsymbol{\beta}-\boldsymbol{\beta}^{*}\right\|_{2} .
$$

Plugging (D.79) into the right-hand side of (D.77), we obtain

$$
5 / 8 \cdot \lambda s^{\prime} \leq\left(\rho_{+}-\zeta_{+}\right) \cdot \sqrt{s^{\prime}}\left\|\boldsymbol{\beta}-\boldsymbol{\beta}^{*}\right\|_{2} .
$$

Plugging the upper bound of $\left\|\boldsymbol{\beta}-\boldsymbol{\beta}^{*}\right\|_{2}$ in Lemma 5.3 of Wang et al. (2014a) into the right-hand side of (D.80), we obtain

$$
\begin{aligned}
\sqrt{s^{\prime}} & \leq \frac{8}{5 \lambda} \cdot\left(\rho_{+}-\zeta_{+}\right)\left\|\boldsymbol{\beta}-\boldsymbol{\beta}^{*}\right\|_{2} \\
& \leq \frac{8}{5 \lambda} \cdot\left(\rho_{+}-\zeta_{+}\right) \cdot \frac{15 / 2}{\rho_{-}-\zeta_{-}} \lambda \sqrt{s^{*}}=12 \kappa \sqrt{s^{*}}
\end{aligned}
$$

where the last equality follows from the definition of the condition number $\kappa$ 
in (4.5) of Wang et al. (2014a). Hence we obtain $s^{\prime} \leq 144 \kappa^{2} s^{*}$. Note that $S^{\prime}$ is defined as an arbitrary subset of $\left\{j:\left|\left(\nabla \widetilde{\mathcal{L}}_{\lambda}(\boldsymbol{\beta})-\nabla \widetilde{\mathcal{L}}_{\lambda}\left(\boldsymbol{\beta}^{*}\right)\right)_{j}\right| \geq 5 / 8 \cdot \lambda\right\}$ and

$$
\begin{aligned}
& \left\{j \in \overline{S^{*}}:\left|\left(\nabla \widetilde{\mathcal{L}}_{\lambda}(\boldsymbol{\beta})-\nabla \widetilde{\mathcal{L}}_{\lambda}\left(\boldsymbol{\beta}^{*}\right)\right)_{j}\right| \geq 5 / 8 \cdot \lambda\right\} \\
& \quad \subseteq\left\{j:\left|\left(\nabla \widetilde{\mathcal{L}}_{\lambda}(\boldsymbol{\beta})-\nabla \widetilde{\mathcal{L}}_{\lambda}\left(\boldsymbol{\beta}^{*}\right)\right)_{j}\right| \geq 5 / 8 \cdot \lambda\right\} .
\end{aligned}
$$

Hence we have

$$
\left|\left\{j \in \overline{S^{*}}:\left|\left(\nabla \widetilde{\mathcal{L}_{\lambda}}(\boldsymbol{\beta}) / L-\nabla \widetilde{\mathcal{L}}_{\lambda}\left(\boldsymbol{\beta}^{*}\right) / L\right)_{j}\right| \geq 5 / 8 \cdot \lambda / L\right\}\right| \leq 144 \kappa^{2} s^{*} .
$$

Therefore, by setting $\widetilde{s}_{3}=144 \kappa^{2} s^{*}$, we obtain (D.59).

In summary, we prove that (D.58)-(D.59) hold with $\widetilde{s}_{1}=250 \kappa s^{*}, \widetilde{s}_{2}=0$ and $\widetilde{s}_{2}=144 \kappa^{2} s^{*}$. In Assumption 4.4 , we assume $\widetilde{s} \geq 144 \kappa^{2}+250 \kappa$, which implies $\widetilde{s}_{1}+\widetilde{s}_{2}+\widetilde{s}_{3} \leq \widetilde{s}$. Therefore we have $\left\|\left(\mathcal{T}_{L, \lambda}(\boldsymbol{\beta} ;+\infty)\right)_{\overline{S^{*}}}\right\|_{0}<\widetilde{s}$. Since $\mathcal{T}_{L, \lambda}(\boldsymbol{\beta} ; R)$ has the same sparsity pattern as $\mathcal{T}_{L, \lambda}(\boldsymbol{\beta} ;+\infty)$, we also have that $\left\|\left(\mathcal{T}_{L, \lambda}(\boldsymbol{\beta} ; R)\right)_{\overline{S^{*}}}\right\|_{0}<\widetilde{s}$ for $R \in(0,+\infty)$. Hence we conclude the proof.

D.6. Proof of Theorem 5.5 in Wang et al. (2014a). We first provide a useful lemma. It states that if $\boldsymbol{\beta}$ is $\epsilon$-suboptimal with respect to the regularization parameter $\lambda$ and sufficiently sparse, then for $\lambda^{\prime} \leq \lambda$ the objective function value $\phi_{\lambda^{\prime}}(\boldsymbol{\beta})$ is close to $\phi_{\lambda^{\prime}}\left(\widehat{\boldsymbol{\beta}}_{\lambda^{\prime}}\right)$. Here $\widehat{\boldsymbol{\beta}}_{\lambda^{\prime}}$ is the exact local solution corresponding to $\lambda^{\prime}$.

Lemma D.3. Let $\lambda \geq \lambda_{\text {tgt }}$ and $\lambda^{\prime} \in\left[\lambda_{\text {tgt }}, \lambda\right]$. Suppose $\left\|\boldsymbol{\beta}_{\overline{S^{*}}}\right\|_{0} \leq \widetilde{s}$ and $\omega_{\lambda}(\boldsymbol{\beta}) \leq \epsilon$. Let $\widehat{\boldsymbol{\beta}}_{\lambda^{\prime}}$ be the exact local solution corresponding to $\lambda^{\prime}$, which satisfies the exact optimality condition in (3.13) of Wang et al. (2014a) and $\left\|\left(\widehat{\boldsymbol{\beta}}_{\lambda^{\prime}}\right)_{\overline{S^{*}}}\right\|_{0} \leq \widetilde{s}$. For logistic loss, we further assume $\max \left\{\|\boldsymbol{\beta}\|_{2},\left\|\widehat{\boldsymbol{\beta}}_{\lambda^{\prime}}\right\|_{2}\right\} \leq$ $R$, where $R$ is specified in Definition 4.3 of Wang et al. (2014a). Under Assumption 4.1 and Assumption 4.4 of Wang et al. (2014a), we have

$$
\phi_{\lambda^{\prime}}(\boldsymbol{\beta})-\phi_{\lambda^{\prime}}\left(\widehat{\boldsymbol{\beta}}_{\lambda^{\prime}}\right) \leq C\left(\epsilon+2\left(\lambda-\lambda^{\prime}\right)\right) \cdot\left(\lambda^{\prime}+\lambda\right) s^{*}, \quad \text { where } C=\frac{21}{\rho_{-}-\zeta_{-}} .
$$

Proof. Since $\left\|\boldsymbol{\beta}_{\overline{S^{*}}}\right\|_{0} \leq \widetilde{s}$ and $\left\|\left(\widehat{\boldsymbol{\beta}}_{\lambda^{\prime}}\right)_{\overline{S^{*}}}\right\|_{0} \leq \widetilde{s}$, we have $\left\|\left(\boldsymbol{\beta}-\widehat{\boldsymbol{\beta}}_{\lambda^{\prime}}\right)_{\overline{S^{*}}}\right\| \leq$ $2 \widetilde{s}$. In the setting of logistic loss, we further have $\|\boldsymbol{\beta}\|_{2} \leq R$ and $\left\|\widehat{\boldsymbol{\beta}}_{\lambda^{\prime}}\right\|_{2} \leq R$. Therefore, Lemma 5.1 of Wang et al. (2014a) gives

$$
\begin{aligned}
\widetilde{\mathcal{L}}_{\lambda^{\prime}}\left(\widehat{\boldsymbol{\beta}}_{\lambda^{\prime}}\right) & \geq \widetilde{\mathcal{L}}_{\lambda^{\prime}}(\boldsymbol{\beta})+\left(\widehat{\boldsymbol{\beta}}_{\lambda^{\prime}}-\boldsymbol{\beta}\right)^{T} \nabla \widetilde{\mathcal{L}}_{\lambda^{\prime}}(\boldsymbol{\beta})+\frac{\rho_{-}-\zeta_{-}}{2}\left\|\widehat{\boldsymbol{\beta}}_{\lambda^{\prime}}-\boldsymbol{\beta}\right\|_{2}^{2} \\
& \geq \widetilde{\mathcal{L}}_{\lambda^{\prime}}(\boldsymbol{\beta})+\left(\widehat{\boldsymbol{\beta}}_{\lambda^{\prime}}-\boldsymbol{\beta}\right)^{T} \nabla \widetilde{\mathcal{L}}_{\lambda^{\prime}}(\boldsymbol{\beta}),
\end{aligned}
$$

where the second inequality is because $\rho_{-}-\zeta_{-}>0$, which follows from (4.3) in Wang et al. (2014a). 
Let $\boldsymbol{\xi} \in \partial\|\boldsymbol{\beta}\|_{1}$ be the subgradient that attains the minimum in

$$
\omega_{\lambda}(\boldsymbol{\beta})=\min _{\boldsymbol{\xi}^{\prime} \in \partial\|\boldsymbol{\beta}\|_{1}} \max _{\boldsymbol{\beta}^{\prime} \in \Omega}\left\{\frac{\left(\boldsymbol{\beta}-\boldsymbol{\beta}^{\prime}\right)^{T}}{\left\|\boldsymbol{\beta}-\boldsymbol{\beta}^{\prime}\right\|_{1}}\left(\nabla \widetilde{\mathcal{L}}_{\lambda}(\boldsymbol{\beta})+\lambda \boldsymbol{\xi}^{\prime}\right)\right\},
$$

where $\Omega=B_{2}(R)$ in the setting of logistic loss and $\Omega=\mathbb{R}^{d}$ in other settings. Since $\boldsymbol{\xi}$ is a minimizer, we have

$$
\omega_{\lambda}(\boldsymbol{\beta})=\max _{\boldsymbol{\beta}^{\prime} \in \Omega}\left\{\frac{\left(\boldsymbol{\beta}-\boldsymbol{\beta}^{\prime}\right)^{T}}{\left\|\boldsymbol{\beta}-\boldsymbol{\beta}^{\prime}\right\|_{1}}\left(\nabla \widetilde{\mathcal{L}}_{\lambda}(\boldsymbol{\beta})+\lambda \boldsymbol{\xi}\right)\right\} .
$$

By the convexity of $\ell_{1}$ norm, we also have

$$
\lambda^{\prime}\left\|\widehat{\boldsymbol{\beta}}_{\lambda^{\prime}}\right\|_{1} \geq \lambda^{\prime}\|\boldsymbol{\beta}\|_{1}+\lambda^{\prime} \boldsymbol{\xi}^{T}\left(\widehat{\boldsymbol{\beta}}_{\lambda^{\prime}}-\boldsymbol{\beta}\right) .
$$

Recall that the objective function $\phi_{\lambda}(\boldsymbol{\beta})$ is defined as $\phi_{\lambda}(\boldsymbol{\beta})=\widetilde{\mathcal{L}}_{\lambda}(\boldsymbol{\beta})+\lambda\|\boldsymbol{\beta}\|_{1}$. Adding (D.82) and (D.85), we obtain

$$
\phi_{\lambda^{\prime}}\left(\widehat{\boldsymbol{\beta}}_{\lambda^{\prime}}\right) \geq \phi_{\lambda^{\prime}}(\boldsymbol{\beta})+\left(\nabla \widetilde{\mathcal{L}}_{\lambda^{\prime}}(\boldsymbol{\beta})+\lambda^{\prime} \boldsymbol{\xi}\right)^{T}\left(\widehat{\boldsymbol{\beta}}_{\lambda^{\prime}}-\boldsymbol{\beta}\right) .
$$

Hence we have

$$
\begin{aligned}
& \phi_{\lambda^{\prime}}(\boldsymbol{\beta})-\phi_{\lambda^{\prime}}\left(\widehat{\boldsymbol{\beta}}_{\lambda^{\prime}}\right) \\
& \leq\left(\nabla \widetilde{\mathcal{L}}_{\lambda^{\prime}}(\boldsymbol{\beta})+\lambda^{\prime} \boldsymbol{\xi}\right)^{T}\left(\boldsymbol{\beta}-\widehat{\boldsymbol{\beta}}_{\lambda^{\prime}}\right) \\
& =(\overbrace{}^{\nabla \mathcal{L}(\boldsymbol{\beta})+\nabla \widetilde{\mathcal{L}}_{\lambda}(\boldsymbol{\beta})}) \\
& \leq \underbrace{\left(\nabla \widetilde{\mathcal{L}}_{\lambda}(\boldsymbol{\beta})+\lambda \boldsymbol{\xi}\right)^{T}\left(\boldsymbol{\beta}-\widehat{\boldsymbol{\beta}}_{\lambda^{\prime}}\right)}_{(\mathrm{i})}+\underbrace{\left\|\nabla \mathcal{Q}_{\lambda^{\prime}}(\boldsymbol{\beta})-\nabla \mathcal{Q}_{\lambda}(\boldsymbol{\beta})\right\|_{\infty}}_{(\mathrm{ii})} \underbrace{\left\|\boldsymbol{\beta}-\widehat{\boldsymbol{\beta}}_{\lambda^{\prime}}\right\|_{1}}_{(\mathrm{iv})} \\
& +\underbrace{\left\|\lambda^{\prime} \boldsymbol{\xi}-\lambda \boldsymbol{\xi}\right\|_{\infty}}_{(\mathrm{iii})} \underbrace{\left\|\boldsymbol{\beta}-\widehat{\boldsymbol{\beta}}_{\lambda^{\prime}}\right\|_{1}}_{(\mathrm{iv})} .
\end{aligned}
$$

Now we provide upper bounds of terms (i)-(iv) correspondingly.

Bounding Term (i) in (D.86): According to (D.84), we have

$$
\frac{\left(\boldsymbol{\beta}-\widehat{\boldsymbol{\beta}}_{\lambda^{\prime}}\right)^{T}}{\left\|\boldsymbol{\beta}-\widehat{\boldsymbol{\beta}}_{\lambda^{\prime}}\right\|_{1}}\left(\nabla \widetilde{\mathcal{L}}_{\lambda}(\boldsymbol{\beta})+\lambda \boldsymbol{\xi}\right) \leq \max _{\boldsymbol{\beta}^{\prime} \in \Omega}\left\{\frac{\left(\boldsymbol{\beta}-\boldsymbol{\beta}^{\prime}\right)^{T}}{\left\|\boldsymbol{\beta}-\boldsymbol{\beta}^{\prime}\right\|_{1}}\left(\nabla \widetilde{\mathcal{L}}_{\lambda}(\boldsymbol{\beta})+\lambda \boldsymbol{\xi}\right)\right\}=\omega_{\lambda}(\boldsymbol{\beta}) \leq \epsilon,
$$

where the last inequality is our assumption. Therefore we obtain

$$
\left(\nabla \widetilde{\mathcal{L}}_{\lambda}(\boldsymbol{\beta})+\lambda \boldsymbol{\xi}\right)^{T}\left(\boldsymbol{\beta}-\widehat{\boldsymbol{\beta}}_{\lambda^{\prime}}\right) \leq \epsilon \cdot\left\|\boldsymbol{\beta}-\widehat{\boldsymbol{\beta}}_{\lambda^{\prime}}\right\|_{1} .
$$

We will provide an upper bound of $\left\|\boldsymbol{\beta}-\widehat{\boldsymbol{\beta}}_{\lambda^{\prime}}\right\|_{1}$ when we handle term (iv). 
Bounding Term (ii) in (D.86): Recall $\mathcal{Q}_{\lambda}(\boldsymbol{\beta})=\sum_{i=1}^{d} q_{\lambda}\left(\beta_{j}\right)$. We have

$$
\begin{aligned}
\left\|\nabla \mathcal{Q}_{\lambda^{\prime}}(\boldsymbol{\beta})-\nabla \mathcal{Q}_{\lambda}(\boldsymbol{\beta})\right\|_{\infty} & =\max _{1 \leq j \leq d}\left|q_{\lambda^{\prime}}\left(\beta_{j}\right)-q_{\lambda}\left(\beta_{j}\right)\right| \\
& \leq \max _{1 \leq j \leq d}\left|\lambda^{\prime}-\lambda\right|=\lambda-\lambda^{\prime}
\end{aligned}
$$

where the inequality follows from regularity condition (e), the last equality is because $\lambda \geq \lambda^{\prime}$.

Bounding Term (iii) in (D.86): Since $\boldsymbol{\xi} \in \partial\|\boldsymbol{\beta}\|_{1}$, we have $\|\boldsymbol{\xi}\|_{\infty} \leq 1$. Then we obtain

$$
\left\|\lambda^{\prime} \boldsymbol{\xi}-\lambda \boldsymbol{\xi}\right\|_{\infty}=\left|\lambda^{\prime}-\lambda\right|\|\boldsymbol{\xi}\|_{\infty} \leq\left|\lambda-\lambda^{\prime}\right|=\lambda-\lambda^{\prime} .
$$

Bounding Term (iv) in (D.86): Note that

$$
\left\|\boldsymbol{\beta}-\widehat{\boldsymbol{\beta}}_{\lambda^{\prime}}\right\|_{1} \leq \underbrace{\left\|\boldsymbol{\beta}-\boldsymbol{\beta}^{*}\right\|_{1}}_{\text {(iv).a }}+\underbrace{\left\|\widehat{\boldsymbol{\beta}}_{\lambda^{\prime}}-\boldsymbol{\beta}^{*}\right\|_{1}}_{\text {(iv).b }} .
$$

For term (iv).a, since $\boldsymbol{\beta}$ satisfies $\left\|\boldsymbol{\beta}_{\overline{S^{*}}}\right\|_{0} \leq \widetilde{s}, \omega_{\lambda}(\boldsymbol{\beta}) \leq \lambda / 2$, and $\|\boldsymbol{\beta}\|_{2} \leq R$ for logistic loss, we have that $\boldsymbol{\beta}$ satisfies the assumptions of Lemma 5.2 in Wang et al. (2014a). Following the same way we obtain (D.37) in the proof of Lemma 5.2 in Wang et al. (2014a), we can get

$$
(\lambda / 2-\lambda / 8)\left\|\left(\boldsymbol{\beta}-\boldsymbol{\beta}^{*}\right)_{\overline{S^{*}}}\right\|_{1} \leq(3 \lambda / 2+\lambda / 8+\lambda)\left\|\left(\boldsymbol{\beta}-\boldsymbol{\beta}^{*}\right)_{S^{*}}\right\|_{1},
$$

which implies $\left\|\left(\boldsymbol{\beta}-\boldsymbol{\beta}^{*}\right)_{S^{*}}\right\|_{1} \leq 7\left\|\left(\boldsymbol{\beta}-\boldsymbol{\beta}^{*}\right)_{S^{*}}\right\|_{1}$. Hence we obtain

$$
\begin{aligned}
\left\|\boldsymbol{\beta}-\boldsymbol{\beta}^{*}\right\|_{1} \leq\left\|\left(\boldsymbol{\beta}-\boldsymbol{\beta}^{*}\right)_{S^{*}}\right\|_{1}+\left\|\left(\boldsymbol{\beta}-\boldsymbol{\beta}^{*}\right)_{S^{*}}\right\|_{1} & \leq 8\left\|\left(\boldsymbol{\beta}-\boldsymbol{\beta}^{*}\right)_{S^{*}}\right\|_{1} \\
& \leq 8 \sqrt{s^{*}}\left\|\left(\boldsymbol{\beta}-\boldsymbol{\beta}^{*}\right)_{S^{*}}\right\|_{2} \\
& \leq 8 \sqrt{s^{*}}\left\|\boldsymbol{\beta}-\boldsymbol{\beta}^{*}\right\|_{2} .
\end{aligned}
$$

With the upper bound of $\left\|\boldsymbol{\beta}-\boldsymbol{\beta}^{*}\right\|_{2}$ in Lemma 5.2 of Wang et al. (2014a), we obtain

$$
\left\|\boldsymbol{\beta}-\boldsymbol{\beta}^{*}\right\|_{1} \leq \frac{21}{\rho_{-}-\zeta_{-}} \lambda s^{*} .
$$

Meanwhile, for term (iv).b, note that we assume $\widehat{\boldsymbol{\beta}}_{\lambda^{\prime}}$ satisfies $\left\|\left(\widehat{\boldsymbol{\beta}}_{\lambda^{\prime}}\right)_{\overline{S^{*}}}\right\|_{0} \leq \widetilde{s}$ and $\left\|\widehat{\boldsymbol{\beta}}_{\lambda^{\prime}}\right\|_{2} \leq R$ for logistic loss. Since $\widehat{\boldsymbol{\beta}}_{\lambda^{\prime}}$ is an exact local solution, it satisfies the exact optimality condition $\omega\left(\widehat{\boldsymbol{\beta}}_{\lambda^{\prime}}\right) \leq 0$, which gives $\omega\left(\widehat{\boldsymbol{\beta}}_{\lambda^{\prime}}\right)<\lambda^{\prime} / 2$. Hence $\widehat{\boldsymbol{\beta}}_{\lambda^{\prime}}$ also satisfies the conditions of Lemma 5.2 of Wang et al. (2014a). Similar to (D.91), we have

$$
\left\|\widehat{\boldsymbol{\beta}}_{\lambda^{\prime}}-\boldsymbol{\beta}^{*}\right\|_{1} \leq \frac{21}{\rho_{-}-\zeta_{-}} \lambda^{\prime} s^{*}
$$


Plugging (D.92) and (D.91) into (D.90), for term (iv) in (D.86), we obtain

$$
\left\|\boldsymbol{\beta}-\widehat{\boldsymbol{\beta}}_{\lambda^{\prime}}\right\|_{1} \leq \frac{21}{\rho_{-}-\zeta_{-}}\left(\lambda^{\prime}+\lambda\right) s^{*} .
$$

Plugging (D.87)-(D.89) and (D.93) into the right-hand side of (D.86), we obtain

$$
\begin{aligned}
& \phi_{\lambda^{\prime}}(\boldsymbol{\beta})-\phi_{\lambda^{\prime}}\left(\widehat{\boldsymbol{\beta}}_{\lambda^{\prime}}\right) \\
& \leq \underbrace{\epsilon \cdot \frac{21}{\rho_{-}-\zeta_{-}}\left(\lambda^{\prime}+\lambda\right) s^{*}}_{(\mathrm{i}) \text { in }(\mathrm{D} .86)}+(\underbrace{\left(\lambda-\lambda^{\prime}\right)}_{\text {(ii) in }(\mathrm{D} .86)}+\underbrace{\left(\lambda-\lambda^{\prime}\right)}_{\text {(iii) in }(\mathrm{D} .86)}) \cdot \underbrace{\frac{21}{\rho_{-}-\zeta_{-}}\left(\lambda^{\prime}+\lambda\right) s^{*}}_{(\mathrm{iv}) \text { in }(\mathrm{D} .86)} \\
& \leq \frac{21}{\rho_{-}-\zeta_{-}}\left(\epsilon+2\left(\lambda-\lambda^{\prime}\right)\right) \cdot\left(\lambda^{\prime}+\lambda\right) s^{*},
\end{aligned}
$$

where the upper bound of term (i) in (D.86) is obtained by plugging (D.93) into the right-hand side of (D.87). Hence we conclude the proof.

Now we are ready to prove Theorem 5.5 of Wang et al. (2014a).

Proof. Sparsity of $\left\{\boldsymbol{\beta}_{t}^{k}\right\}_{k=0}^{\infty}$ within the $t$-th Stage: In the following, we provide results concerning the sparsity of the sequence $\left\{\boldsymbol{\beta}_{t}^{k}\right\}_{k=0}^{\infty}$ within the $t$-th path following stage. In the following we prove this by induction. Note that the initialization satisfies

$$
\left\|\left(\boldsymbol{\beta}_{t}^{0}\right)_{\overline{S^{*}}}\right\|_{0} \leq \widetilde{s}, \quad \omega_{\lambda_{t}}\left(\boldsymbol{\beta}_{t}^{0}\right) \leq \lambda_{t} / 2, \quad \text { and } \quad L_{t}^{0} \leq 2\left(\rho_{+}-\zeta_{+}\right) .
$$

By Lemma 5.2 of Wang et al. (2014a) we have

$$
\phi_{\lambda_{t}}\left(\boldsymbol{\beta}_{t}^{0}\right)-\phi_{\lambda_{t}}\left(\boldsymbol{\beta}^{*}\right) \leq \frac{21 / 2}{\rho_{-}-\zeta_{-}} \lambda_{t}^{2} s^{*},
$$

Suppose that, at the $(k-1)$-th iteration of the proximal-gradient method (Lines 5-9 of Algorithm 3 in Wang et al. (2014a)), we have

$$
\left\|\left(\boldsymbol{\beta}_{t}^{k-1}\right)_{\overline{S^{*}}}\right\|_{0} \leq \widetilde{s}, L_{t}^{k-1} \leq 2\left(\rho_{+}-\zeta_{+}\right), \phi_{\lambda_{t}}\left(\boldsymbol{\beta}_{t}^{k-1}\right)-\phi_{\lambda_{t}}\left(\boldsymbol{\beta}^{*}\right) \leq \frac{21 / 2}{\rho_{-}-\zeta_{-}} \lambda_{t}^{2} s^{*},
$$

Then by Lemma 5.4 in Wang et al. (2014a), we have that $\boldsymbol{\beta}_{t}^{k}=\mathcal{T}_{L_{t}^{k}, \lambda_{t}}\left(\boldsymbol{\beta}_{t}^{k-1} ; R\right)$ satisfies

$$
\left\|\left(\boldsymbol{\beta}_{t}^{k}\right)_{\overline{S^{*}}}\right\|_{0} \leq \widetilde{s}
$$

Note that, in the setting of logistic loss, we always have $\left\|\boldsymbol{\beta}_{t}^{k}\right\|_{2} \leq R$ for $k=0,1, \ldots$ because of the $\ell_{2}$ constraint $\Omega=B_{2}(R)$. Since $\left\|\left(\boldsymbol{\beta}_{t}^{k-1}\right)_{\overline{S^{*}}}\right\|_{0} \leq \widetilde{s}$ and $\left\|\left(\boldsymbol{\beta}_{t}^{k}\right)_{\overline{S^{*}}}\right\|_{0} \leq \widetilde{s}$ imply $\left\|\left(\boldsymbol{\beta}_{t}^{k-1}-\boldsymbol{\beta}_{t}^{k}\right)_{\overline{S^{*}}}\right\| \leq 2 \widetilde{s}$, from Lemma 5.1 of Wang 
et al. (2014a) we have

$$
\begin{aligned}
\widetilde{\mathcal{L}}_{\lambda_{t}}\left(\boldsymbol{\beta}_{t}^{k}\right) \geq \widetilde{\mathcal{L}}_{\lambda_{t}}\left(\boldsymbol{\beta}_{t}^{k-1}\right)+\nabla \widetilde{\mathcal{L}}_{\lambda_{t}}\left(\boldsymbol{\beta}_{t}^{k-1}\right)^{T}\left(\boldsymbol{\beta}_{t}^{k}-\boldsymbol{\beta}_{t}^{k-1}\right) & \\
& +\frac{\rho_{-}-\zeta_{-}}{2}\left\|\boldsymbol{\beta}_{t}^{k}-\boldsymbol{\beta}_{t}^{k-1}\right\|_{2}^{2}, \\
\widetilde{\mathcal{L}}_{\lambda_{t}}\left(\boldsymbol{\beta}_{t}^{k}\right) \leq \widetilde{\mathcal{L}}_{\lambda_{t}}\left(\boldsymbol{\beta}_{t}^{k-1}\right)+\nabla \widetilde{\mathcal{L}}_{\lambda_{t}}\left(\boldsymbol{\beta}_{t}^{k-1}\right)^{T} & \left(\boldsymbol{\beta}_{t}^{k}-\boldsymbol{\beta}_{t}^{k-1}\right) \\
& +\frac{\rho_{+}-\zeta_{+}}{2}\left\|\boldsymbol{\beta}_{t}^{k}-\boldsymbol{\beta}_{t}^{k-1}\right\|_{2}^{2}
\end{aligned}
$$

Now we prove that (D.99) guarantees the line-search method in Algorithm 2 of Wang et al. (2014a) produces $L_{t}^{k} \leq 2\left(\rho_{+}-\zeta_{+}\right)$. We prove by contradiction: We assume that, when the line-search method stops, it outputs $L_{t}^{k}>2\left(\rho_{+}-\zeta_{+}\right)$. Recall that we double $L_{t}^{k}$ at each line-search iteration (Line 6 of Algorithm 2 in Wang et al. (2014a)). Then at the line-search iteration right before the line-search method stops, we have $L_{t}^{k^{\prime}}=L_{t}^{k} / 2>\left(\rho_{+}-\zeta_{+}\right)$. Recall that the objective function $\phi_{\lambda}(\boldsymbol{\beta})=\widetilde{\mathcal{L}}_{\lambda}(\boldsymbol{\beta})+\lambda\|\boldsymbol{\beta}\|_{1}$. Adding $\lambda_{t}\left\|\boldsymbol{\beta}_{t}^{k}\right\|_{1}$ to the both sides of (D.99), we obtain

$$
\begin{aligned}
\phi_{\lambda_{t}}\left(\boldsymbol{\beta}_{t}^{k}\right)= & \widetilde{\mathcal{L}}_{\lambda_{t}}\left(\boldsymbol{\beta}_{t}^{k}\right)+\lambda_{t}\left\|\boldsymbol{\beta}_{t}^{k}\right\|_{1} \\
\leq & \widetilde{\mathcal{L}}_{\lambda_{t}}\left(\boldsymbol{\beta}_{t}^{k-1}\right)+\nabla \widetilde{\mathcal{L}}_{\lambda_{t}}\left(\boldsymbol{\beta}_{t}^{k-1}\right)^{T}\left(\boldsymbol{\beta}_{t}^{k}-\boldsymbol{\beta}_{t}^{k-1}\right) \\
\quad & \quad+\frac{\rho_{+}-\zeta_{+}}{2}\left\|\boldsymbol{\beta}_{t}^{k}-\boldsymbol{\beta}_{t}^{k-1}\right\|_{2}^{2}+\lambda_{t}\left\|\boldsymbol{\beta}_{t}^{k}\right\|_{1} \\
& \leq \widetilde{\mathcal{L}}_{\lambda_{t}}\left(\boldsymbol{\beta}_{t}^{k-1}\right)+\nabla \widetilde{\mathcal{L}}_{\lambda_{t}}\left(\boldsymbol{\beta}_{t}^{k-1}\right)^{T}\left(\boldsymbol{\beta}_{t}^{k}-\boldsymbol{\beta}_{t}^{k-1}\right) \\
\quad & \quad+\frac{L_{t}^{k^{\prime}}}{2}\left\|\boldsymbol{\beta}_{t}^{k}-\boldsymbol{\beta}_{t}^{k-1}\right\|_{2}^{2}+\lambda_{t}\left\|\boldsymbol{\beta}_{t}^{k}\right\|_{1} \\
= & \psi_{L_{t}^{k^{\prime}}, \lambda_{t}}\left(\boldsymbol{\beta}_{t}^{k} ; \boldsymbol{\beta}_{t}^{k-1}\right),
\end{aligned}
$$

where the last equality follows from (3.7) of Wang et al. (2014a). The stopping criterion of Algorithm 2 in Wang et al. (2014a) implies that the line-search method should have already stopped and give $\left(L_{t}^{k}\right)^{\prime}=L_{t}^{k} / 2$, which contradicts our assumption that the line-search method outputs $L_{t}^{k}$. Therefore we have

$$
L_{t}^{k} \leq 2\left(\rho_{+}-\zeta_{+}\right)
$$

Moreover, according to (D.98) and (D.99), Lemma D.1 holds, i.e.,

$$
\phi_{\lambda_{t}}\left(\boldsymbol{\beta}_{t}^{k}\right) \leq \phi_{\lambda_{t}}\left(\boldsymbol{\beta}_{t}^{k-1}\right)-\frac{L_{t}^{k}}{2}\left\|\boldsymbol{\beta}_{t}^{k}-\boldsymbol{\beta}_{t}^{k-1}\right\|_{2}^{2}
$$


which implies

$$
\begin{aligned}
\phi_{\lambda_{t}}\left(\boldsymbol{\beta}_{t}^{k}\right)-\phi_{\lambda_{t}}\left(\boldsymbol{\beta}^{*}\right) & \leq \phi_{\lambda_{t}}\left(\boldsymbol{\beta}_{t}^{k-1}\right)-\frac{L_{t}^{k}}{2}\left\|\boldsymbol{\beta}_{t}^{k}-\boldsymbol{\beta}_{t}^{k-1}\right\|_{2}^{2}-\phi_{\lambda_{t}}\left(\boldsymbol{\beta}^{*}\right) \\
& \leq \frac{21 / 2}{\rho_{-}-\zeta_{-}} \lambda_{t}^{2} s^{*}
\end{aligned}
$$

According to (D.97) and (D.100)-(D.102), now we have

$$
\left\|\left(\boldsymbol{\beta}_{t}^{k}\right)_{\bar{S}^{*}}\right\|_{0} \leq \widetilde{s}, \quad L_{t}^{k} \leq 2\left(\rho_{+}-\zeta_{+}\right), \quad \phi_{\lambda_{t}}\left(\boldsymbol{\beta}_{t}^{k}\right)-\phi_{\lambda_{t}}\left(\boldsymbol{\beta}^{*}\right) \leq \frac{21 / 2}{\rho_{-}-\zeta_{-}} \lambda_{t}^{2} s^{*} .
$$

Combining (D.94), (D.96) and (D.103), by induction we prove that (D.103) holds for all $k=0,1, \ldots$ within the $t$-th path following stage. Furthermore, by Lemma 5.3 of Wang et al. (2014a), all $\boldsymbol{\beta}_{t}^{k}$ 's have nice statistical recovery properties, i.e.,

$$
\left\|\boldsymbol{\beta}_{t}^{k}-\boldsymbol{\beta}^{*}\right\|_{2} \leq \frac{15 / 2}{\rho_{-}-\zeta_{-}} \lambda_{t} \sqrt{s^{*}}, \quad \text { for } k=0,1, \ldots
$$

Convergence to Unique Local Solution: In the following, we prove that, within the $t$-th path following stage, the limit point of the sequence $\left\{\boldsymbol{\beta}_{t}^{k}\right\}_{k=0}^{\infty}$ generated by Algorithm 3 in Wang et al. (2014a) is unique and also an exact local solution. Since $\left\|\left(\boldsymbol{\beta}_{t}^{0}\right)_{\overline{S^{*}}}\right\| \leq \widetilde{s}$, the restricted strong convexity of $\widetilde{\mathcal{L}}_{\lambda}(\boldsymbol{\beta})$ in Lemma 5.1 of Wang et al. (2014a) implies that the sub-level set

$$
\left\{\boldsymbol{\beta}: \phi_{\lambda_{t}}(\boldsymbol{\beta}) \leq \phi_{\lambda_{t}}\left(\boldsymbol{\beta}_{t}^{0}\right),\left\|\left(\boldsymbol{\beta}_{t}^{0}-\boldsymbol{\beta}\right)_{\overline{S^{*}}}\right\| \leq 2 \widetilde{s}\right\}
$$

is bounded. From (D.101) and (D.103) we have

$$
\phi_{\lambda_{t}}\left(\boldsymbol{\beta}_{t}^{k}\right) \leq \phi_{\lambda_{t}}\left(\boldsymbol{\beta}_{t}^{0}\right) \text { and }\left\|\left(\boldsymbol{\beta}_{t}^{k}\right)_{\overline{S^{*}}}\right\|_{0} \leq \widetilde{s}, \quad \text { for } k=1,2, \ldots
$$

Thus $\left\{\boldsymbol{\beta}_{t}^{k}\right\}_{k=0}^{\infty}$ is bounded, which implies that $\left\{\phi_{\lambda_{t}}\left(\boldsymbol{\beta}_{t}^{k}\right)\right\}_{k=0}^{\infty}$ is also bounded. Meanwhile, (D.101) implies that $\left\{\phi_{\lambda_{t}}\left(\boldsymbol{\beta}_{t}^{k}\right)\right\}_{k=0}^{\infty}$ decreases monotonically. By the Bolzano-Weierstrass theorem, the limit point of $\left\{\phi_{\lambda_{t}}\left(\boldsymbol{\beta}_{t}^{k}\right)\right\}_{k=0}^{\infty}$ is unique, which implies

$$
\lim _{k \rightarrow \infty}\left\{\phi_{\lambda_{t}}\left(\boldsymbol{\beta}_{t}^{k}\right)-\phi_{\lambda_{t}}\left(\boldsymbol{\beta}_{t}^{k-1}\right)\right\}=0 .
$$

Consequently, by (D.101) we have that, for any limit point of $\left\{\boldsymbol{\beta}^{k}\right\}_{k=0}^{\infty}$,

$$
\lim _{k \rightarrow \infty}\left\{\left\|\boldsymbol{\beta}_{t}^{k}-\boldsymbol{\beta}_{t}^{k-1}\right\|_{2}\right\} \leq \frac{2}{L_{t}^{k}} \cdot \lim _{k \rightarrow \infty}\left\{\phi_{\lambda_{t}}\left(\boldsymbol{\beta}_{t}^{k}\right)-\phi_{\lambda_{t}}\left(\boldsymbol{\beta}_{t}^{k-1}\right)\right\}=0 .
$$

Moreover, Lemma D.2 implies

$$
\lim _{k \rightarrow \infty}\left\{\omega_{\lambda_{t}}\left(\boldsymbol{\beta}_{t}^{k}\right)\right\} \leq\left(L_{t}^{k}+\left(\rho_{+}-\zeta_{+}\right)\right) \cdot \lim _{k \rightarrow \infty}\left\{\left\|\boldsymbol{\beta}_{t}^{k}-\boldsymbol{\beta}_{t}^{k-1}\right\|_{2}\right\}=0 .
$$


In other words, the sequence $\left\{\boldsymbol{\beta}_{t}^{k}\right\}_{k=0}^{\infty}$ has a convergent subsequence, which satisfies $\lim _{k \rightarrow \infty}\left\{\omega_{\lambda_{t}}\left(\boldsymbol{\beta}_{t}^{k}\right)\right\} \leq 0$. Furthermore, it implies that this convergent subsequence of $\left\{\boldsymbol{\beta}_{t}^{k}\right\}_{k=0}^{\infty}$ converges towards an exact local solution $\widehat{\boldsymbol{\beta}}_{\lambda_{t}}$ that satisfies the optimal condition in (3.13) of Wang et al. (2014a). By (D.103) we have $\left\|\left(\boldsymbol{\beta}_{t}^{k}\right)_{\overline{S^{*}}}\right\|_{0} \leq \widetilde{s}(k=1,2, \ldots)$, which implies $\left\|\left(\widehat{\boldsymbol{\beta}}_{\lambda_{t}}\right)_{\overline{S^{*}}}\right\|_{0} \leq \widetilde{s}$.

Now we prove the uniqueness of this exact local solution by contradiction. Let $\boldsymbol{\xi} \in \partial\left\|\widehat{\boldsymbol{\beta}}_{\lambda_{t}}\right\|_{1}$ be the subgradient that attains the minimum in

$$
\omega_{\lambda_{t}}\left(\widehat{\boldsymbol{\beta}}_{\lambda_{t}}\right)=\min _{\boldsymbol{\xi}^{\prime} \in \partial\left\|\widehat{\boldsymbol{\beta}}_{\lambda_{t}}\right\|_{1}} \max _{\boldsymbol{\beta}^{\prime} \in \Omega}\left\{\frac{\left(\widehat{\boldsymbol{\beta}}_{\lambda_{t}}-\boldsymbol{\beta}^{\prime}\right)^{T}}{\left\|\widehat{\boldsymbol{\beta}}_{\lambda_{t}}-\boldsymbol{\beta}^{\prime}\right\|_{1}}\left(\nabla \widetilde{\mathcal{L}}_{\lambda_{t}}\left(\widehat{\boldsymbol{\beta}}_{\lambda_{t}}\right)+\lambda_{t} \boldsymbol{\xi}^{\prime}\right)\right\} .
$$

Since $\omega_{\lambda_{t}}\left(\widehat{\boldsymbol{\beta}}_{\lambda_{t}}\right) \leq 0$, we have

$$
\max _{\boldsymbol{\beta}^{\prime} \in \Omega}\left\{\frac{\left(\widehat{\boldsymbol{\beta}}_{\lambda_{t}}-\boldsymbol{\beta}^{\prime}\right)^{T}}{\left\|\widehat{\boldsymbol{\beta}}_{\lambda_{t}}-\boldsymbol{\beta}^{\prime}\right\|_{1}}\left(\nabla \widetilde{\mathcal{L}}_{\lambda_{t}}\left(\widehat{\boldsymbol{\beta}}_{\lambda_{t}}\right)+\lambda_{t} \boldsymbol{\xi}\right)\right\} \leq 0 .
$$

We assume there exists another local solution $\widehat{\boldsymbol{\beta}}_{\lambda_{t}}^{\prime}$, which is the limit point of another convergent subsequence of $\left\{\boldsymbol{\beta}_{t}^{k}\right\}_{k=0}^{\infty}$. Since $\left\|\left(\widehat{\boldsymbol{\beta}}_{\lambda_{t}}^{\prime}\right){\overline{S^{*}}}_{0}\right\|_{0} \leq \widetilde{s}$, we have $\left\|\left(\widehat{\boldsymbol{\beta}}_{\lambda_{t}}^{\prime}-\widehat{\boldsymbol{\beta}}_{\lambda_{t}}\right)_{\overline{S^{*}}}\right\| \leq 2 \widetilde{s}$. In the setting of logistic loss, we have $\left\|\widehat{\boldsymbol{\beta}}_{\lambda_{t}}^{\prime}\right\|_{2} \leq R$ and $\left\|\widehat{\boldsymbol{\beta}}_{\lambda_{t}}\right\|_{2} \leq R$ by the $\ell_{2}$ constraint. Hence Lemma 5.1 of Wang et al. (2014a) implies

$$
\begin{aligned}
\widetilde{\mathcal{L}}_{\lambda_{t}}\left(\widehat{\boldsymbol{\beta}}_{\lambda_{t}}^{\prime}\right) \geq \widetilde{\mathcal{L}}_{\lambda_{t}}\left(\widehat{\boldsymbol{\beta}}_{\lambda_{t}}\right)+\left(\widehat{\boldsymbol{\beta}}_{\lambda_{t}}^{\prime}\right. & \left.-\widehat{\boldsymbol{\beta}}_{\lambda_{t}}\right)^{T} \nabla \widetilde{\mathcal{L}}_{\lambda_{t}}\left(\widehat{\boldsymbol{\beta}}_{\lambda_{t}}\right) \\
& +\frac{\rho-\zeta_{-}}{2}\left\|\widehat{\boldsymbol{\beta}}_{\lambda_{t}}^{\prime}-\widehat{\boldsymbol{\beta}}_{\lambda_{t}}\right\|_{2}^{2} .
\end{aligned}
$$

Meanwhile, the convexity of $\ell_{1}$ norm implies

$$
\lambda_{t}\left\|\widehat{\boldsymbol{\beta}}_{\lambda_{t}}^{\prime}\right\|_{1} \geq \lambda_{t}\left\|\widehat{\boldsymbol{\beta}}_{\lambda_{t}}\right\|_{1}+\lambda_{t}\left(\widehat{\boldsymbol{\beta}}_{\lambda_{t}}^{\prime}-\widehat{\boldsymbol{\beta}}_{\lambda_{t}}\right)^{T} \boldsymbol{\xi}
$$

Recall that the objective function $\phi_{\lambda}(\boldsymbol{\beta})=\widetilde{\mathcal{L}}_{\lambda}(\boldsymbol{\beta})+\lambda\|\boldsymbol{\beta}\|_{1}$. Adding (D.106) and (D.107), we obtain

$$
\begin{aligned}
& \phi_{\lambda_{t}}\left(\widehat{\boldsymbol{\beta}}_{\lambda_{t}}^{\prime}\right)-\phi_{\lambda_{t}}\left(\widehat{\boldsymbol{\beta}}_{\lambda_{t}}\right) \\
& \geq \underbrace{\left(\nabla \widetilde{\mathcal{L}}_{\lambda_{t}}\left(\widehat{\boldsymbol{\beta}}_{\lambda_{t}}\right)+\lambda_{t} \boldsymbol{\xi}\right)^{T}\left(\widehat{\boldsymbol{\beta}}_{\lambda_{t}}^{\prime}-\widehat{\boldsymbol{\beta}}_{\lambda_{t}}\right)}+\frac{\rho_{-}-\zeta_{-}}{2}\left\|\widehat{\boldsymbol{\beta}}_{\lambda_{t}}^{\prime}-\widehat{\boldsymbol{\beta}}_{\lambda_{t}}\right\|_{2}^{2} .
\end{aligned}
$$

Since (D.105) implies

$$
\begin{aligned}
\frac{\left(\widehat{\boldsymbol{\beta}}_{\lambda_{t}}-\widehat{\boldsymbol{\beta}}_{\lambda_{t}}^{\prime}\right)^{T}}{\left\|\widehat{\boldsymbol{\beta}}_{\lambda_{t}}-\widehat{\boldsymbol{\beta}}_{\lambda_{t}}^{\prime}\right\|_{1}}\left(\nabla \widetilde{\mathcal{L}}_{\lambda_{t}}\left(\widehat{\boldsymbol{\beta}}_{\lambda_{t}}\right)+\lambda_{t} \boldsymbol{\xi}\right) & \leq \max _{\boldsymbol{\beta}^{\prime} \in \Omega}\left\{\frac{\left(\widehat{\boldsymbol{\beta}}_{\lambda_{t}}-\boldsymbol{\beta}^{\prime}\right)^{T}}{\left\|\widehat{\boldsymbol{\beta}}_{\lambda_{t}}-\boldsymbol{\beta}^{\prime}\right\|_{1}}\left(\nabla \widetilde{\mathcal{L}}_{\lambda_{t}}\left(\widehat{\boldsymbol{\beta}}_{\lambda_{t}}\right)+\lambda_{t} \boldsymbol{\xi}\right)\right\} \\
& \leq 0
\end{aligned}
$$


term (i) in (D.108) is nonnegative. Hence we obtain

$$
\phi_{\lambda_{t}}\left(\widehat{\boldsymbol{\beta}}_{\lambda_{t}}^{\prime}\right)-\phi_{\lambda_{t}}\left(\widehat{\boldsymbol{\beta}}_{\lambda_{t}}\right) \geq \frac{\rho_{-}-\zeta_{-}}{2}\left\|\widehat{\boldsymbol{\beta}}_{\lambda_{t}}^{\prime}-\widehat{\boldsymbol{\beta}}_{\lambda_{t}}\right\|_{2}^{2}
$$

Recall we already know that the limit point of $\left\{\phi_{\lambda_{t}}\left(\boldsymbol{\beta}_{t}^{k}\right)\right\}_{k=0}^{\infty}$ is unique, which implies $\phi_{\lambda_{t}}\left(\widehat{\boldsymbol{\beta}}_{\lambda_{t}}^{\prime}\right)-\phi_{\lambda_{t}}\left(\widehat{\boldsymbol{\beta}}_{\lambda_{t}}\right)=0$. Then we obtain $\left\|\widehat{\boldsymbol{\beta}}_{\lambda_{t}}^{\prime}-\widehat{\boldsymbol{\beta}}_{\lambda_{t}}\right\|_{2}^{2}=0$, which contradicts our assumption that $\widehat{\boldsymbol{\beta}}_{\lambda_{t}}^{\prime} \neq \widehat{\boldsymbol{\beta}}_{\lambda_{t}}$. In other words, we prove that the sequence $\left\{\boldsymbol{\beta}_{t}^{k}\right\}_{k=0}^{\infty}$ converges to a unique local solution $\widehat{\boldsymbol{\beta}}_{\lambda_{t}}$.

Geometric Convergence Rate of Algorithm 3 in Wang et al. (2014a): Now we establish the geometric rate of convergence of Algorithm 3. According to the stopping criterion of Algorithm 2 in Wang et al. (2014a), we have

$$
\begin{aligned}
\phi_{\lambda_{t}}\left(\boldsymbol{\beta}_{t}^{k}\right) \leq & \psi_{L_{t}^{k}, \lambda_{t}}\left(\boldsymbol{\beta}_{t}^{k} ; \boldsymbol{\beta}_{t}^{k-1}\right) \\
& =\min _{\boldsymbol{\beta}}\left\{\widetilde{\mathcal{L}}_{\lambda_{t}}\left(\boldsymbol{\beta}_{t}^{k-1}\right)+\nabla \widetilde{\mathcal{L}}_{\lambda_{t}}\left(\boldsymbol{\beta}_{t}^{k-1}\right)^{T}\left(\boldsymbol{\beta}-\boldsymbol{\beta}_{t}^{k-1}\right)\right. \\
& \left.+\frac{L_{t}^{k}}{2}\left\|\boldsymbol{\beta}-\boldsymbol{\beta}_{t}^{k-1}\right\|_{2}^{2}+\lambda_{t}\|\boldsymbol{\beta}\|_{1}\right\}
\end{aligned}
$$

$$
\begin{array}{r}
\leq \min _{\substack{\boldsymbol{\beta}=\alpha \widehat{\boldsymbol{\beta}}_{\lambda_{t}}+(1-\alpha) \boldsymbol{\beta}_{t}^{k-1} \\
\alpha \in[0,1]}}\{\overbrace{\widetilde{\mathcal{L}}_{\lambda_{t}}\left(\boldsymbol{\beta}_{t}^{k-1}\right)+\nabla \widetilde{\mathcal{L}}_{\lambda_{t}}\left(\boldsymbol{\beta}_{t}^{k-1}\right)^{T}\left(\boldsymbol{\beta}-\boldsymbol{\beta}_{t}^{k-1}\right)} \\
\left.+\frac{L_{t}^{k}}{2}\left\|\boldsymbol{\beta}-\boldsymbol{\beta}_{t}^{k-1}\right\|_{2}^{2}+\lambda_{t}\|\boldsymbol{\beta}\|_{1}\right\} .
\end{array}
$$

For term (i), since $\left\|\left(\boldsymbol{\beta}_{t}^{k-1}\right)_{\overline{S^{*}}}\right\|_{0} \leq \widetilde{s},\left\|\left(\widehat{\boldsymbol{\beta}}_{\lambda_{t}}\right)_{\overline{S^{*}}}\right\|_{0} \leq \widetilde{s}$ and $\boldsymbol{\beta}=\alpha \widehat{\boldsymbol{\beta}}_{\lambda_{t}}+(1-$ $\alpha) \boldsymbol{\beta}_{t}^{k-1}$ with $\alpha \in[0,1]$, we obtain $\left\|\left(\boldsymbol{\beta}-\boldsymbol{\beta}_{t}^{k-1}\right)_{S^{*}}\right\|_{0} \leq 2 \widetilde{s}$. For logistic loss, since $\left\|\boldsymbol{\beta}_{t}^{k-1}\right\|_{2} \leq R$ and $\left\|\widehat{\boldsymbol{\beta}}_{\lambda_{t}}\right\|_{2} \leq R$, we have $\|\boldsymbol{\beta}\|_{2} \leq R$, since the $\ell_{2}$ ball $B_{2}(R)$ is a convex set. Applying Lemma 5.1 in Wang et al. (2014a), we have

$$
\begin{aligned}
\widetilde{\mathcal{L}}_{\lambda_{t}}(\boldsymbol{\beta}) \geq \widetilde{\mathcal{L}}_{\lambda_{t}}\left(\boldsymbol{\beta}_{t}^{k-1}\right)+\nabla \widetilde{\mathcal{L}}_{\lambda_{t}}\left(\boldsymbol{\beta}_{t}^{k-1}\right)^{T}\left(\boldsymbol{\beta}-\boldsymbol{\beta}_{t}^{k-1}\right) \\
\quad+\frac{\rho_{-}-\zeta_{-}}{2}\left\|\boldsymbol{\beta}-\boldsymbol{\beta}_{t}^{k-1}\right\|_{2}^{2} \\
\geq \widetilde{\mathcal{L}}_{\lambda_{t}}\left(\boldsymbol{\beta}_{t}^{k-1}\right)+\nabla \widetilde{\mathcal{L}}_{\lambda_{t}}\left(\boldsymbol{\beta}_{t}^{k-1}\right)^{T}\left(\boldsymbol{\beta}-\boldsymbol{\beta}_{t}^{k-1}\right),
\end{aligned}
$$

where the second inequality follows from (4.3) in Wang et al. (2014a). Plugging (D.111) into (D.110), we obtain

$$
\phi_{\lambda_{t}}\left(\boldsymbol{\beta}_{t}^{k}\right) \leq \min _{\substack{\boldsymbol{\beta}=\alpha \widehat{\boldsymbol{\beta}}_{\lambda_{t}}+(1-\alpha) \boldsymbol{\beta}_{t}^{k-1} \\ \alpha \in[0,1]}}\left\{\widetilde{\mathcal{L}}_{\lambda_{t}}(\boldsymbol{\beta})+\frac{L_{t}^{k}}{2}\left\|\boldsymbol{\beta}-\boldsymbol{\beta}_{t}^{k-1}\right\|_{2}^{2}+\lambda_{t}\|\boldsymbol{\beta}\|_{1}\right\}
$$


Since $\left\|\left(\boldsymbol{\beta}_{t}^{k-1}\right)_{\overline{S^{*}}}\right\|_{0} \leq \widetilde{s}$ and $\left\|\left(\widehat{\boldsymbol{\beta}}_{\lambda_{t}}\right)_{\overline{S^{*}}}\right\|_{0} \leq \widetilde{s}$ imply $\left\|\left(\widehat{\boldsymbol{\beta}}_{\lambda_{t}}-\boldsymbol{\beta}_{t}^{k-1}\right)_{\overline{S^{*}}}\right\|_{0} \leq 2 \widetilde{s}$, Lemma 5.1 in Wang et al. (2014a) implies that the strong convexity of $\widetilde{\mathcal{L}}_{\lambda_{t}}(\boldsymbol{\beta})$ holds for $\widehat{\boldsymbol{\beta}}_{\lambda_{t}}$ and $\boldsymbol{\beta}_{t}^{k-1}$. Hence we have

$$
\begin{aligned}
\widetilde{\mathcal{L}}_{\lambda_{t}}(\boldsymbol{\beta}) & =\widetilde{\mathcal{L}}_{\lambda_{t}}\left(\alpha \widehat{\boldsymbol{\beta}}_{\lambda_{t}}+(1-\alpha) \boldsymbol{\beta}^{k-1}\right) \\
& \leq \alpha \widetilde{\mathcal{L}}_{\lambda_{t}}\left(\widehat{\boldsymbol{\beta}}_{\lambda_{t}}\right)+(1-\alpha) \widetilde{\mathcal{L}}_{\lambda_{t}}\left(\boldsymbol{\beta}^{k-1}\right)
\end{aligned}
$$

Meanwhile, by the convexity of $\ell_{1}$ norm we have

$$
\begin{aligned}
\lambda_{t}\|\boldsymbol{\beta}\|_{1} & =\lambda_{t}\left\|\alpha \widehat{\boldsymbol{\beta}}_{\lambda_{t}}+(1-\alpha) \boldsymbol{\beta}^{k-1}\right\|_{1} \\
& \leq \alpha \lambda_{t}\left\|\widehat{\boldsymbol{\beta}}_{\lambda_{t}}\right\|_{1}+(1-\alpha)\left\|\boldsymbol{\beta}^{k-1}\right\|_{1} .
\end{aligned}
$$

Plugging (D.113) and (D.114) into the right-hand side of (D.112), we obtain

$$
\begin{aligned}
\phi_{\lambda_{t}}\left(\boldsymbol{\beta}_{t}^{k}\right) \leq \min _{\alpha \in[0,1]}\left\{\alpha \left(\widetilde{\mathcal{L}}_{\lambda_{t}}\left(\widehat{\boldsymbol{\beta}}_{\lambda_{t}}\right)\right.\right. & \left.+\lambda_{t}\left\|\widehat{\boldsymbol{\beta}}_{\lambda_{t}}\right\|_{1}\right) \\
& +(1-\alpha)\left(\widetilde{\mathcal{L}}_{\lambda_{t}}\left(\boldsymbol{\beta}_{t}^{k-1}\right)+\lambda_{t}\left\|\boldsymbol{\beta}_{t}^{k-1}\right\|_{1}\right) \\
& \left.+\frac{L_{t}^{k}}{2}\left\|\alpha \widehat{\boldsymbol{\beta}}_{\lambda_{t}}+(1-\alpha) \boldsymbol{\beta}_{t}^{k-1}-\boldsymbol{\beta}_{t}^{k-1}\right\|_{2}^{2}\right\} \\
=\min _{\alpha \in[0,1]}\left\{\alpha \phi_{\lambda_{t}}\left(\widehat{\boldsymbol{\beta}}_{\lambda_{t}}\right)\right. & +(1-\alpha) \phi_{\lambda_{t}}\left(\boldsymbol{\beta}_{t}^{k-1}\right) \\
& \left.+\frac{L_{t}^{k}}{2}\left\|\alpha \widehat{\boldsymbol{\beta}}_{\lambda_{t}}+(1-\alpha) \boldsymbol{\beta}_{t}^{k-1}-\boldsymbol{\beta}_{t}^{k-1}\right\|_{2}^{2}\right\} \\
\leq \min _{\alpha \in[0,1]}\left\{\phi_{\lambda_{t}}\left(\boldsymbol{\beta}_{t}^{k-1}\right)-\alpha\left(\phi_{\lambda_{t}}\left(\boldsymbol{\beta}_{t}^{k-1}\right)-\phi_{\lambda_{t}}\left(\widehat{\boldsymbol{\beta}}_{\lambda_{t}}\right)\right)\right. & +\frac{\alpha^{2} L_{t}^{k}}{2} \underbrace{\left\|\boldsymbol{\beta}_{t}^{k-1}-\widehat{\boldsymbol{\beta}}_{\lambda_{t}}\right\|_{2}^{2}}\} .
\end{aligned}
$$

For term (i), similar to (D.109), applying the exact optimality condition of $\widehat{\boldsymbol{\beta}}_{\lambda_{t}}$ and the restricted strong convexity of $\widetilde{\mathcal{L}}_{\lambda_{t}}(\boldsymbol{\beta})$, we obtain

$$
\phi_{\lambda_{t}}\left(\boldsymbol{\beta}_{t}^{k-1}\right)-\phi_{\lambda_{t}}\left(\widehat{\boldsymbol{\beta}}_{\lambda_{t}}\right) \geq \frac{\rho_{-}-\zeta_{-}}{2}\left\|\boldsymbol{\beta}_{t}^{k-1}-\widehat{\boldsymbol{\beta}}_{\lambda_{t}}\right\|_{2}^{2} .
$$

Plugging this into the right-hand side of (D.115), we obtain

$$
\begin{aligned}
\phi_{\lambda_{t}}\left(\boldsymbol{\beta}_{t}^{k}\right) \leq \min _{\alpha \in[0,1]}\left\{\phi_{\lambda_{t}}\left(\boldsymbol{\beta}_{t}^{k-1}\right)-\alpha\left(\phi_{\lambda_{t}}\left(\boldsymbol{\beta}_{t}^{k-1}\right)-\phi_{\lambda_{t}}\left(\widehat{\boldsymbol{\beta}}_{\lambda_{t}}\right)\right)\right. \\
\left.\quad+\frac{\alpha^{2} L_{t}^{k}}{2} \cdot \frac{2}{\rho_{-}-\zeta_{-}}\left(\phi_{\lambda_{t}}\left(\boldsymbol{\beta}_{t}^{k-1}\right)-\phi_{\lambda_{t}}\left(\widehat{\boldsymbol{\beta}}_{\lambda_{t}}\right)\right)\right\} .
\end{aligned}
$$

The right-hand side of (D.116) attains its minimum if $\alpha=\left(\rho_{-}-\zeta_{-}\right) /\left(2 L_{t}^{k}\right)$. 
Plugging this value of $\alpha$ into (D.116), we obtain

$$
\phi_{\lambda_{t}}\left(\boldsymbol{\beta}_{t}^{k}\right) \leq \phi_{\lambda_{t}}\left(\boldsymbol{\beta}_{t}^{k-1}\right)-\frac{\rho_{-}-\zeta_{-}}{4 L_{t}^{k}}\left(\phi_{\lambda_{t}}\left(\boldsymbol{\beta}_{t}^{k-1}\right)-\phi_{\lambda_{t}}\left(\widehat{\boldsymbol{\beta}}_{\lambda_{t}}\right)\right),
$$

which implies

$$
\begin{aligned}
& \phi_{\lambda_{t}}\left(\boldsymbol{\beta}_{t}^{k}\right)-\phi_{\lambda_{t}}\left(\widehat{\boldsymbol{\beta}}_{\lambda_{t}}\right) \\
& \leq\left(\phi_{\lambda_{t}}\left(\boldsymbol{\beta}_{t}^{k-1}\right)-\phi_{\lambda_{t}}\left(\widehat{\boldsymbol{\beta}}_{\lambda_{t}}\right)\right)-\frac{\rho_{-}-\zeta_{-}}{4 L_{t}^{k}}\left(\phi_{\lambda_{t}}\left(\boldsymbol{\beta}_{t}^{k-1}\right)-\phi_{\lambda_{t}}\left(\widehat{\boldsymbol{\beta}}_{\lambda_{t}}\right)\right) \\
& =\left(1-\frac{\rho_{-}-\zeta_{-}}{4 L_{t}^{k}}\right)\left(\phi_{\lambda_{t}}\left(\boldsymbol{\beta}_{t}^{k-1}\right)-\phi_{\lambda_{t}}\left(\widehat{\boldsymbol{\beta}}_{\lambda_{t}}\right)\right) .
\end{aligned}
$$

Recall that in (D.103) we have $L_{t}^{k} \leq 2\left(\rho_{+}-\zeta_{+}\right)(k=0,1, \ldots)$. Plugging in this into the right-hand side of (D.117), we obtain

$$
\begin{aligned}
\phi_{\lambda_{t}}\left(\boldsymbol{\beta}_{t}^{k}\right)-\phi_{\lambda_{t}}\left(\widehat{\boldsymbol{\beta}}_{\lambda_{t}}\right) \leq & (1-\frac{1}{8} \cdot \underbrace{\frac{\rho_{-}-\zeta_{-}}{\rho_{+}-\zeta_{t}}}_{1 / \kappa})\left(\phi_{\lambda_{t}}\left(\boldsymbol{\beta}_{t}^{k-1}\right)-\phi_{\lambda_{t}}\left(\widehat{\boldsymbol{\beta}}_{\lambda_{t}}\right)\right) \\
= & \left(1-\frac{1}{8 \kappa}\right)^{2}\left(\phi_{\lambda_{t}}\left(\boldsymbol{\beta}_{t}^{k-2}\right)-\phi_{\lambda_{t}}\left(\widehat{\boldsymbol{\beta}}_{\lambda_{t}}\right)\right) \\
\vdots & \\
= & \left(1-\frac{1}{8 \kappa}\right)^{k}\left(\phi_{\lambda_{t}}\left(\boldsymbol{\beta}_{t}^{0}\right)-\phi_{\lambda_{t}}\left(\widehat{\boldsymbol{\beta}}_{\lambda_{t}}\right)\right),
\end{aligned}
$$

where $\kappa$ is the condition number defined in (4.5) of Wang et al. (2014a). Now we can characterize the total number of proximal-gradient steps required to obtain an approximate solution $\widetilde{\boldsymbol{\beta}}_{t}=\boldsymbol{\beta}_{t}^{k+1}$ that satisfies

$$
\omega_{\lambda_{t}}\left(\widetilde{\boldsymbol{\beta}}_{t}\right) \leq \lambda_{t} / 4 \quad(t=1, \ldots, N-1), \quad \text { or } \quad \omega_{\lambda_{t}}(\widetilde{\boldsymbol{\beta}}) \leq \epsilon_{\mathrm{opt}} \quad(t=N)
$$

From Lemma D.2, we have

$$
\begin{aligned}
\omega_{\lambda_{t}}\left(\boldsymbol{\beta}_{t}^{k+1}\right) & \leq\left(L_{t}^{k+1}+\left(\rho_{+}-\zeta_{+}\right)\right)\left\|\boldsymbol{\beta}_{t}^{k+1}-\boldsymbol{\beta}_{t}^{k}\right\|_{2} \\
& =L_{t}^{k+1}\left(1+\frac{\rho_{+}-\zeta_{+}}{L_{t}^{k+1}}\right)\left\|\boldsymbol{\beta}_{t}^{k+1}-\boldsymbol{\beta}_{t}^{k}\right\|_{2} .
\end{aligned}
$$

Note that the stopping criterion of the line-search method (Line 7 of Algorithm 2 in in Wang et al. (2014a)) implies $L_{t}^{k+1} \geq \rho_{-}-\zeta_{-}$. Otherwise, we assume that $L_{t}^{k+1}<\rho_{-}-\zeta_{-}$. Since $\left\|\left(\boldsymbol{\beta}_{t}^{k+1}\right)_{\overline{S^{*}}}\right\|_{0} \leq \widetilde{s}$ and $\left\|\left(\boldsymbol{\beta}_{t}^{k}\right)_{\overline{S^{*}}}\right\|_{0} \leq \widetilde{s}$ 
imply $\left\|\left(\boldsymbol{\beta}_{t}^{k}-\boldsymbol{\beta}_{t}^{k+1}\right)_{\overline{S^{*}}}\right\|_{0} \leq 2 \widetilde{s}$, by Lemma 5.1 in Wang et al. (2014a) we have

$$
\begin{aligned}
& \psi_{L_{t}^{k+1}, \lambda_{t}}\left(\boldsymbol{\beta}_{t}^{k+1} ; \boldsymbol{\beta}_{t}^{k}\right) \\
& =\widetilde{\mathcal{L}}_{\lambda_{t}}\left(\boldsymbol{\beta}_{t}^{k}\right)+\nabla \widetilde{\mathcal{L}}_{\lambda_{t}}\left(\boldsymbol{\beta}_{t}^{k}\right)^{T}\left(\boldsymbol{\beta}_{t}^{k+1}-\boldsymbol{\beta}_{t}^{k}\right)+\frac{L^{k+1}}{2}\left\|\boldsymbol{\beta}_{t}^{k+1}-\boldsymbol{\beta}_{t}^{k}\right\|_{2}^{2} \\
& +\lambda_{t}\left\|\boldsymbol{\beta}_{t}^{k+1}\right\|_{1} \\
& <\widetilde{\mathcal{L}}_{\lambda_{t}}\left(\boldsymbol{\beta}_{t}^{k}\right)+\nabla \widetilde{\mathcal{L}}_{\lambda_{t}}\left(\boldsymbol{\beta}_{t}^{k}\right)^{T}\left(\boldsymbol{\beta}_{t}^{k+1}-\boldsymbol{\beta}_{t}^{k}\right)+\frac{\rho_{-}-\zeta_{-}}{2}\left\|\boldsymbol{\beta}_{t}^{k+1}-\boldsymbol{\beta}_{t}^{k}\right\|_{2}^{2} \\
& \leq \lambda_{t}\left\|\boldsymbol{\beta}_{t}^{k+1}\right\|_{1} \\
& =\widetilde{\mathcal{L}}_{\lambda_{t}}\left(\boldsymbol{\beta}_{t}^{k+1}\right)+\lambda_{t}\left\|\boldsymbol{\beta}_{t}^{k+1}\right\|_{1} \\
& =\phi_{\lambda_{t}}\left(\boldsymbol{\beta}_{t}^{k+1}\right) .
\end{aligned}
$$

Here the first equality is from the definition in (3.7) of Wang et al. (2014a), the first inequality is from our assumption that $L_{t}^{k+1}<\rho_{-}-\zeta_{-}$, the second inequality follows from the restricted strong convexity by Lemma 5.1 in Wang et al. (2014a). However, this contradicts the stopping criterion $\phi_{\lambda_{t}}\left(\boldsymbol{\beta}_{t}^{k+1}\right) \leq$ $\psi_{L_{t}^{k+1}, \lambda_{t}}\left(\boldsymbol{\beta}_{t}^{k+1} ; \boldsymbol{\beta}_{t}^{k}\right)$. Therefore we have proved $L_{t}^{k+1} \geq \rho_{-}-\zeta_{-}$. From (D.120) we have

$$
\begin{aligned}
\omega_{\lambda_{t}}\left(\boldsymbol{\beta}_{t}^{k+1}\right) & \leq L_{t}^{k+1}\left(1+\frac{\rho_{+}-\zeta_{+}}{\rho_{-}-\zeta_{-}}\right)\left\|\boldsymbol{\beta}_{t}^{k+1}-\boldsymbol{\beta}_{t}^{k}\right\|_{2} \\
& =L_{t}^{k+1}(1+\kappa)\left\|\boldsymbol{\beta}_{t}^{k+1}-\boldsymbol{\beta}_{t}^{k}\right\|_{2} .
\end{aligned}
$$

Moreover, by Lemma D.1 we have

$$
\frac{L_{t}^{k+1}}{2}\left\|\boldsymbol{\beta}_{t}^{k+1}-\boldsymbol{\beta}_{t}^{k}\right\|_{2}^{2} \leq \phi_{\lambda}\left(\boldsymbol{\beta}_{t}^{k}\right)-\phi_{\lambda}\left(\boldsymbol{\beta}_{t}^{k+1}\right) .
$$

Plugging this into the right-hand side of (D.121), we obtain

$$
\begin{aligned}
\omega_{\lambda_{t}}\left(\boldsymbol{\beta}_{t}^{k+1}\right) & \leq(1+\kappa) L_{t}^{k+1}\left\|\boldsymbol{\beta}_{t}^{k+1}-\boldsymbol{\beta}_{t}^{k}\right\|_{2} \\
& \leq(1+\kappa) \sqrt{2 L_{t}^{k+1}\left(\phi_{\lambda_{t}}\left(\boldsymbol{\beta}_{t}^{k}\right)-\phi_{\lambda_{t}}\left(\boldsymbol{\beta}_{t}^{k+1}\right)\right)} .
\end{aligned}
$$

According to (D.101), the sequence $\left\{\phi_{\lambda_{t}}\left(\boldsymbol{\beta}_{t}^{k}\right)\right\}_{k=0}^{\infty}$ decreases monotonically. Therefore, we have $\phi_{\lambda_{t}}\left(\boldsymbol{\beta}_{t}^{k+1}\right) \geq \phi_{\lambda_{t}}\left(\widehat{\boldsymbol{\beta}}_{\lambda_{t}}\right)$, which implies

$$
\omega_{\lambda_{t}}\left(\boldsymbol{\beta}_{t}^{k+1}\right) \leq(1+\kappa) \sqrt{2 L_{t}^{k+1}\left(\phi_{\lambda_{t}}\left(\boldsymbol{\beta}_{t}^{k}\right)-\phi_{\lambda_{t}}\left(\widehat{\boldsymbol{\beta}}_{\lambda_{t}}\right)\right)}
$$

Now we provide an upper bound of the right-hand side of (D.122). Recall that in (D.103) we have $L_{t}^{k} \leq 2\left(\rho_{+}-\zeta_{+}\right)(k=0,1, \ldots)$, and in (D.118) we have $\phi_{\lambda_{t}}\left(\boldsymbol{\beta}_{t}^{k}\right)-\phi_{\lambda_{t}}\left(\widehat{\boldsymbol{\beta}}_{\lambda_{t}}\right) \leq(1-1 /(8 \kappa))^{k}\left(\phi_{\lambda_{t}}\left(\boldsymbol{\beta}_{t}^{0}\right)-\phi_{\lambda_{t}}\left(\widehat{\boldsymbol{\beta}}_{\lambda_{t}}\right)\right)$. Note that we assume $\left\|\left(\boldsymbol{\beta}_{t}^{0}\right)_{\overline{S^{*}}}\right\|_{0} \leq \widetilde{s}$ and $\omega_{\lambda_{t}}\left(\boldsymbol{\beta}_{t}^{0}\right) \leq \lambda_{t} / 2$. In Lemma D.3, we set 
$\lambda^{\prime}=\lambda=\lambda_{t}$ and $\epsilon=\lambda_{t} / 2$, then we have

$$
\phi_{\lambda_{t}}\left(\boldsymbol{\beta}_{t}^{0}\right)-\phi_{\lambda_{t}}\left(\widehat{\boldsymbol{\beta}}_{\lambda_{t}}\right) \leq \frac{21}{\rho_{-}-\zeta_{-}} \lambda_{t}^{2} s^{*}
$$

Plugging these into the right-hand side of (D.122), we obtain

$$
\begin{aligned}
\omega_{\lambda_{t}}\left(\boldsymbol{\beta}_{t}^{k+1}\right) & \leq(1+\kappa) \sqrt{4\left(\rho_{+}-\zeta_{+}\right) \cdot\left(1-\frac{1}{8 \kappa}\right)^{k} \frac{21}{\rho_{-}-\zeta_{-}} \lambda_{t}^{2} s^{*}} \\
& =(1+\kappa) \sqrt{84 \kappa\left(1-\frac{1}{8 \kappa}\right)^{k}} \cdot \lambda_{t} \sqrt{s^{*}} .
\end{aligned}
$$

Therefore, for $t=1, \ldots, N-1$, to ensure that $\boldsymbol{\beta}_{t}^{k+1}$ satisfies $\omega_{\lambda_{t}}\left(\boldsymbol{\beta}_{t}^{k+1}\right) \leq \lambda_{t} / 4$, it suffices to make $k$ satisfy

$$
(1+\kappa) \sqrt{84 \kappa\left(1-\frac{1}{8 \kappa}\right)^{k}} \cdot \lambda_{t} \sqrt{s^{*}} \leq \lambda_{t} / 4
$$

which implies

$$
k \geq 2 \log \left(8 \sqrt{21} \cdot \sqrt{\kappa}(1+\kappa) \cdot \sqrt{s^{*}}\right) / \log \left(1-\frac{1}{8 \kappa}\right) .
$$

Similarly, for $t=N$, to ensure that $\boldsymbol{\beta}_{t}^{k+1}$ satisfies $\omega_{\lambda_{t}}\left(\boldsymbol{\beta}^{k+1}\right) \leq \epsilon_{\mathrm{opt}}, k$ should satisfy

$$
k \geq 2 \log \left(2 \sqrt{21} \cdot \sqrt{\kappa}(1+\kappa) \cdot \sqrt{s^{*}} \lambda_{t} / \epsilon_{\mathrm{opt}}\right) / \log \left(1-\frac{1}{8 \kappa}\right) .
$$

Therefore we conclude the proof of Theorem 5.5.

D.7. Proof of Theorem 4.5 in Wang et al. (2014a). First we present a useful lemma. It ensures that the approximate solution $\widetilde{\boldsymbol{\beta}}_{t-1}$, which is obtained from the $(t-1)$-th path following stage, is $\left(\lambda_{t} / 2\right)$-suboptimal with respect to regularization parameter $\lambda_{t}$, i.e., $\omega_{\lambda_{t}}\left(\widetilde{\boldsymbol{\beta}}_{t-1}\right) \leq \lambda_{t} / 2$.

Lemma D.4. Let $\widetilde{\boldsymbol{\beta}}_{t-1}(t=1, \ldots, N)$ be the approximate solution obtained from the $(t-1)$-th path following stage (Line 8 of Algorithm 1 in Wang et al. (2014a)). If $\omega_{\lambda_{t-1}}\left(\widetilde{\boldsymbol{\beta}}_{t-1}\right) \leq \lambda_{t-1} / 4$. Under Assumption 4.1 and Assumption 4.4 in Wang et al. (2014a), we have

$$
\omega_{\lambda_{t}}\left(\widetilde{\boldsymbol{\beta}}_{t-1}\right) \leq \lambda_{t} / 2
$$

where $\lambda_{t}=\eta \lambda_{t-1}$ with $\eta \in[0.9,1)$.

Proof. Consider the regularization parameter $\lambda_{t-1}$. Let $\boldsymbol{\xi} \in \partial\left\|\widetilde{\boldsymbol{\beta}}_{t-1}\right\|_{1}$ 
be the subgradient that attains the minimum in

$$
\omega_{\lambda_{t-1}}\left(\widetilde{\boldsymbol{\beta}}_{t-1}\right)=\min _{\boldsymbol{\xi}^{\prime} \in \partial\left\|\widetilde{\boldsymbol{\beta}}_{t-1}\right\|_{1}} \max _{\boldsymbol{\beta}^{\prime} \in \Omega}\left\{\frac{\left(\widetilde{\boldsymbol{\beta}}_{t-1}-\boldsymbol{\beta}^{\prime}\right)^{T}}{\left\|\widetilde{\boldsymbol{\beta}}_{t-1}-\boldsymbol{\beta}^{\prime}\right\|_{1}}\left(\nabla \widetilde{\mathcal{L}}_{\lambda_{t-1}}\left(\widetilde{\boldsymbol{\beta}}_{t-1}\right)+\lambda_{t-1} \boldsymbol{\xi}^{\prime}\right)\right\},
$$

which implies

$$
\omega_{\lambda_{t-1}}\left(\widetilde{\boldsymbol{\beta}}_{t-1}\right)=\max _{\boldsymbol{\beta}^{\prime} \in \Omega}\left\{\frac{\left(\widetilde{\boldsymbol{\beta}}_{t-1}-\boldsymbol{\beta}^{\prime}\right)^{T}}{\left\|\widetilde{\boldsymbol{\beta}}_{t-1}-\boldsymbol{\beta}^{\prime}\right\|_{1}}\left(\nabla \widetilde{\mathcal{L}}_{\lambda_{t-1}}\left(\widetilde{\boldsymbol{\beta}}_{t-1}\right)+\lambda_{t-1} \boldsymbol{\xi}\right)\right\} .
$$

Now we consider regularization parameter $\lambda_{t}$. We have

$$
\begin{aligned}
& \omega_{\lambda_{t}}\left(\widetilde{\boldsymbol{\beta}}_{t-1}\right)=\min _{\boldsymbol{\xi}^{\prime} \in \partial\left\|\widetilde{\boldsymbol{\beta}}_{t-1}\right\|_{1}} \max _{\boldsymbol{\beta}^{\prime} \in \Omega}\left\{\frac{\left(\widetilde{\boldsymbol{\beta}}_{t-1}-\boldsymbol{\beta}^{\prime}\right)^{T}}{\left\|\widetilde{\boldsymbol{\beta}}_{t-1}-\boldsymbol{\beta}^{\prime}\right\|_{1}}\left(\nabla \widetilde{\mathcal{L}}_{\lambda_{t}}\left(\widetilde{\boldsymbol{\beta}}_{t-1}\right)+\lambda_{t} \boldsymbol{\xi}^{\prime}\right)\right\} \\
& \leq \max _{\boldsymbol{\beta}^{\prime} \in \Omega}\left\{\frac{\left(\widetilde{\boldsymbol{\beta}}_{t-1}-\boldsymbol{\beta}^{\prime}\right)^{T}}{\left\|\widetilde{\boldsymbol{\beta}}_{t-1}-\boldsymbol{\beta}^{\prime}\right\|_{1}}\left(\nabla \widetilde{\mathcal{L}}_{\lambda_{t}}\left(\widetilde{\boldsymbol{\beta}}_{t-1}\right)+\lambda_{t} \boldsymbol{\xi}\right)\right\},
\end{aligned}
$$

where $\boldsymbol{\xi}$ is defined as the minimizer of (D.123). Recall that $\nabla \widetilde{\mathcal{L}}_{\lambda_{t}}\left(\widetilde{\boldsymbol{\beta}}_{t-1}\right)=$ $\nabla \mathcal{L}\left(\widetilde{\boldsymbol{\beta}}_{t-1}\right)+\nabla \mathcal{Q}_{\lambda_{t}}\left(\widetilde{\boldsymbol{\beta}}_{t-1}\right)$. We have

$$
\begin{array}{r}
\nabla \widetilde{\mathcal{L}}_{\lambda_{t}}\left(\widetilde{\boldsymbol{\beta}}_{t-1}\right)+\lambda_{t} \boldsymbol{\xi}=\left(\nabla \mathcal{L}\left(\widetilde{\boldsymbol{\beta}}_{t-1}\right)+\nabla \mathcal{Q}_{\lambda_{t-1}}\left(\widetilde{\boldsymbol{\beta}}_{t-1}\right)+\lambda_{t} \boldsymbol{\xi}\right)+\left(\lambda_{t-1} \boldsymbol{\xi}-\lambda_{t} \boldsymbol{\xi}\right) \\
+\left(\nabla \mathcal{Q}_{\lambda_{t}}\left(\widetilde{\boldsymbol{\beta}}_{t-1}\right)-\nabla \mathcal{Q}_{\lambda_{t-1}}\left(\widetilde{\boldsymbol{\beta}}_{t-1}\right)\right) .
\end{array}
$$

Plugging this into the right-hand side of (D.125), we obtain

$$
\begin{aligned}
& \omega_{\lambda_{t}}\left(\widetilde{\boldsymbol{\beta}}_{t-1}\right) \leq \underbrace{\max _{\boldsymbol{\beta}^{\prime} \in \Omega}\left\{\frac{\left(\widetilde{\boldsymbol{\beta}}_{t-1}-\boldsymbol{\beta}^{\prime}\right)^{T}}{\left\|\widetilde{\boldsymbol{\beta}}_{t-1}-\boldsymbol{\beta}^{\prime}\right\|_{1}}\left(\nabla \widetilde{\mathcal{L}}_{\lambda_{t-1}}\left(\widetilde{\boldsymbol{\beta}}_{t-1}\right)+\lambda_{t-1} \boldsymbol{\xi}\right)\right\}} \\
& \text { (i) } \\
& +\underbrace{\max _{\boldsymbol{\beta}^{\prime} \in \Omega}\left\{\frac{\left(\widetilde{\boldsymbol{\beta}}_{t-1}-\boldsymbol{\beta}^{\prime}\right)^{T}}{\left\|\widetilde{\boldsymbol{\beta}}_{t-1}-\boldsymbol{\beta}^{\prime}\right\|_{1}}\left(\lambda_{t-1} \boldsymbol{\xi}-\lambda_{t} \boldsymbol{\xi}\right)\right\}} \\
& \text { (ii) } \\
& +\underbrace{\max _{2}\left\{\frac{\left(\widetilde{\boldsymbol{\beta}}_{t-1}-\boldsymbol{\beta}^{\prime}\right)^{T}}{\left\|\widetilde{\boldsymbol{\beta}}_{t-1}-\boldsymbol{\beta}^{\prime}\right\|_{1}}\left(\nabla \mathcal{Q}_{\lambda_{t}}\left(\widetilde{\boldsymbol{\beta}}_{t-1}\right)-\nabla \mathcal{Q}_{\lambda_{t-1}}\left(\widetilde{\boldsymbol{\beta}}_{t-1}\right)\right)\right\}}_{\left(\boldsymbol{\beta}^{\prime} \in \Omega\right.} .
\end{aligned}
$$

According to (D.124), term (i) in (D.126) is equal to $\omega_{\lambda_{t-1}}\left(\widetilde{\boldsymbol{\beta}}_{t-1}\right)$, which is upper bounded by $\lambda_{t-1} / 4$ by our assumption. For term (ii) in (D.126), we 
have

$$
\begin{aligned}
\max _{\boldsymbol{\beta}^{\prime} \in \Omega}\left\{\frac{\left(\widetilde{\boldsymbol{\beta}}_{t-1}-\boldsymbol{\beta}^{\prime}\right)^{T}}{\left\|\widetilde{\boldsymbol{\beta}}_{t-1}-\boldsymbol{\beta}^{\prime}\right\|_{1}}\left(\lambda_{t-1} \boldsymbol{\xi}-\lambda_{t} \boldsymbol{\xi}\right)\right\} & \leq \max _{\boldsymbol{\beta}^{\prime} \in \mathbb{R}^{d}}\left\{\frac{\left(\widetilde{\boldsymbol{\beta}}_{t-1}-\boldsymbol{\beta}^{\prime}\right)^{T}}{\left\|\widetilde{\boldsymbol{\beta}}_{t-1}-\boldsymbol{\beta}^{\prime}\right\|_{1}}\left(\lambda_{t-1} \boldsymbol{\xi}-\lambda_{t} \boldsymbol{\xi}\right)\right\} \\
& =\left\|\lambda_{t-1} \boldsymbol{\xi}-\lambda_{t} \boldsymbol{\xi}\right\|_{\infty} \\
& \leq \lambda_{t-1}-\lambda_{t},
\end{aligned}
$$

where first inequality is due to the duality between $\ell_{1}$ and $\ell_{\infty}$ norm, while the second inequality is due to the fact that $\lambda_{t-1}>\lambda_{t}$ and $\|\boldsymbol{\xi}\|_{\infty} \leq 1$, which follows from $\boldsymbol{\xi} \in \partial\left\|\widetilde{\boldsymbol{\beta}}_{t-1}\right\|_{1}$. Similarly, for term (iii) we have

$$
\begin{aligned}
& \max _{\boldsymbol{\beta}^{\prime} \in \Omega}\left\{\frac{\left(\widetilde{\boldsymbol{\beta}}_{t-1}-\boldsymbol{\beta}^{\prime}\right)^{T}}{\left\|\widetilde{\boldsymbol{\beta}}_{t-1}-\boldsymbol{\beta}^{\prime}\right\|_{1}}\left(\nabla \mathcal{Q}_{\lambda_{t}}\left(\widetilde{\boldsymbol{\beta}}_{t-1}\right)-\nabla \mathcal{Q}_{\lambda_{t-1}}\left(\widetilde{\boldsymbol{\beta}}_{t-1}\right)\right)\right\} \\
& \leq\left\|\nabla \mathcal{Q}_{\lambda_{t}}\left(\widetilde{\boldsymbol{\beta}}_{t-1}\right)-\nabla \mathcal{Q}_{\lambda_{t-1}}\left(\widetilde{\boldsymbol{\beta}}_{t-1}\right)\right\|_{\infty} \\
& =\max _{1 \leq j \leq d}\left|q_{\lambda_{t}}^{\prime}\left(\left(\widetilde{\beta}_{t-1}\right)_{j}\right)-q_{\lambda_{t-1}}^{\prime}\left(\left(\widetilde{\beta}_{t-1}\right)_{j}\right)\right| \\
& \leq \lambda_{t-1}-\lambda_{t},
\end{aligned}
$$

where the second inequality follows from regularity condition (e) in Wang et al. (2014a). Hence, from (D.126) we obtain

$$
\begin{aligned}
\omega_{\lambda_{t}}\left(\widetilde{\boldsymbol{\beta}}_{t-1}\right) & \leq \overbrace{\lambda_{t-1} / 4}^{\text {(i) in (D.126) }}+\overbrace{\lambda_{t-1}-\lambda_{t}}^{\text {(ii) in (D.126) }}+\overbrace{\lambda_{t-1}-\lambda_{t}}^{\text {(iii) in (D.126) }} \\
& \leq(1 /(4 \eta)+1 / \eta-1+1 / \eta-1) \lambda_{t} \leq \lambda_{t} / 2,
\end{aligned}
$$

where the last inequality is obtained by plugging in $\eta \in[0.9,1)$. Hence we conclude the proof.

Now we are ready to prove Theorem 4.5 in Wang et al. (2014a).

Proof. Geometric Rate of Convergence within Each Stage: The stopping criterion of Algorithm 3 (Line 9) in Wang et al. (2014a) implies

$$
\omega_{\lambda_{t-1}}\left(\widetilde{\boldsymbol{\beta}}_{t-1}\right) \leq \lambda_{t-1} / 4, \text { for } t=1, \ldots, N \text {. }
$$

By Lemma D.4 we have

$$
\omega_{\lambda_{t}}\left(\widetilde{\boldsymbol{\beta}}_{t-1}\right) \leq \lambda_{t} / 2, \quad \text { for } t=1, \ldots, N .
$$

Recall we initialize the $t$-th stage with $\widetilde{\boldsymbol{\beta}}_{t-1}=\boldsymbol{\beta}_{t}^{0}$ and $L_{t-1}=L_{t}^{0}$ (Line 8 of Algorithm 1). By Theorem 5.5 in Wang et al. (2014a), as long as $\left\|\left(\widetilde{\boldsymbol{\beta}}_{t-1}\right)_{\overline{S^{*}}}\right\|_{0} \leq \widetilde{s}$ and $L_{(t-1)} \leq 2\left(\rho_{+}-\zeta_{+}\right)$, we have

$$
\left\|\left(\boldsymbol{\beta}_{t}^{k}\right)_{\overline{S^{*}}}\right\|_{0} \leq \widetilde{s}, \quad L_{t}^{k} \leq 2\left(\rho_{+}-\zeta_{+}\right), \quad \text { for } k=1,2, \ldots,
$$


which implies $\left\|\left(\widetilde{\boldsymbol{\beta}}_{t}\right)_{\bar{S}^{*}}\right\|_{0} \leq \widetilde{s}$ and $L_{t} \leq 2\left(\rho_{+}-\zeta_{+}\right)$. Remind that we initialize the entire path following procedure with $\widetilde{\boldsymbol{\beta}}_{0}=\mathbf{0}$ and $L_{0}=L_{\min } \leq 2\left(\rho_{+}-\zeta_{+}\right)$ (Line 4 of Algorithm 1 in Wang et al. (2014a)). By induction we obtain

$$
\left\|\left(\widetilde{\boldsymbol{\beta}}_{t}\right)_{S^{*}}\right\|_{0} \leq \widetilde{s}, \quad L_{t} \leq 2\left(\rho_{+}-\zeta_{+}\right), \quad \text { for } t=1, \ldots, N
$$

By setting $\lambda=\lambda_{t}$ and $\widetilde{\boldsymbol{\beta}}=\widetilde{\boldsymbol{\beta}}_{t}(t=1, \ldots, N)$ in Theorem 5.5 of Wang et al. (2014a), we obtain that, within the $t$-th stage $(t=1, \ldots, N-1)$, the total number of proximal-gradient iterations is no more than

$$
2 \log \left(8 \sqrt{21} \cdot \sqrt{\kappa}(1+\kappa) \cdot \sqrt{s^{*}}\right) / \log \left(\frac{1}{1-1 /(8 \kappa)}\right),
$$

while within the $N$-th stage, the total number of proximal-gradient steps is no more that

$$
2 \log \left(2 \sqrt{21} \cdot \sqrt{\kappa}(1+\kappa) \cdot \sqrt{s^{*}} \lambda_{\text {tgt }} / \epsilon_{\text {opt }}\right) / \log \left(\frac{1}{1-1 /(8 \kappa)}\right) .
$$

Hence we obtain the first conclusion.

Geometric Rate of Convergence over the Full Path: Now we prove the second statement about the total number of proximal-gradient steps along the entire solution path. The total number of path following stages is

$$
N=\log \left(\lambda_{\text {tgt }} / \lambda_{0}\right) / \log \eta \text {. }
$$

Together with the first result, we have that the total number of proximalgradient steps is no more than

$$
(N-1) C^{\prime} \log \left(4 C \sqrt{s^{*}}\right)+C^{\prime} \log \left(C \sqrt{s^{*}} \lambda_{\text {tgt }} / \epsilon_{\text {opt }}\right) .
$$

where

$$
C=2 \sqrt{21} \cdot \sqrt{\kappa}(1+\kappa), \quad C^{\prime}=2 / \log \left(\frac{1}{1-1 /(8 \kappa)}\right) .
$$

Geometric Rate of Convergence of the Objective Function Values: Now we prove the third statement concerning the objective function value. For $t=1, \ldots, N-1$, by (D.127) we have $\omega_{\lambda_{t+1}}\left(\widetilde{\boldsymbol{\beta}}_{t}\right) \leq \lambda_{t+1} / 2$. Setting $\lambda^{\prime}=\lambda_{\text {tgt }}$, $\lambda=\lambda_{t+1}, \boldsymbol{\beta}=\widetilde{\boldsymbol{\beta}}_{t}$ and $\epsilon=\lambda_{t+1} / 2$ in Lemma D.3, we obtain $\phi_{\lambda_{\mathrm{tgt}}}\left(\widetilde{\boldsymbol{\beta}}_{t}\right)-\phi_{\lambda_{\mathrm{tgt}}}\left(\widehat{\boldsymbol{\beta}}_{\lambda_{\mathrm{tgt}}}\right) \leq \frac{21}{\rho_{-}-\zeta_{-}}\left(\lambda_{t+1} / 2+2\left(\lambda_{t+1}-\lambda_{\mathrm{tgt}}\right)\right) \cdot\left(\lambda_{\mathrm{tgt}}+\lambda_{t+1}\right) s^{*}$.

Since $\lambda_{\text {tgt }} \leq \lambda_{t+1}$, we have

$\phi_{\lambda_{\mathrm{tgt}}}\left(\widetilde{\boldsymbol{\beta}}_{t}\right)-\phi_{\lambda_{\mathrm{tgt}}}\left(\widehat{\boldsymbol{\beta}}_{\lambda_{\mathrm{tgt}}}\right) \leq \frac{21}{\rho_{-}-\zeta_{-}}\left(\lambda_{t+1} / 2+2 \lambda_{t+1}\right) \cdot 2 \lambda_{t+1} s^{*}=\frac{105 \cdot \lambda_{t+1}^{2} s^{*}}{\rho_{-}-\zeta_{-}}$. 
Since $\lambda_{t+1}=\eta^{t+1} \lambda_{0}$, we obtain

$$
\phi_{\lambda_{\mathrm{tgt}}}\left(\widetilde{\boldsymbol{\beta}}_{t}\right)-\phi_{\lambda_{\mathrm{tgt}}}\left(\widehat{\boldsymbol{\beta}}_{\lambda_{\mathrm{tgt}}}\right) \leq \eta^{2(t+1)} \frac{105 \cdot \lambda_{0}^{2} s^{*}}{\rho_{-}-\zeta_{-}}, \text {for } t=1, \ldots, N-1 .
$$

Similarly, for $t=N$, we have $\omega_{\lambda_{\text {tgt }}}\left(\widetilde{\boldsymbol{\beta}}_{N}\right) \leq \epsilon_{\mathrm{opt}}$. By setting $\lambda=\lambda^{\prime}=\lambda_{\text {tgt }}$ and $\epsilon=\epsilon_{\mathrm{opt}}$ in Lemma D.3, we have

$$
\phi_{\lambda_{\mathrm{tgt}}}\left(\widetilde{\boldsymbol{\beta}}_{t}\right)-\phi_{\lambda_{\mathrm{tgt}}}\left(\widehat{\boldsymbol{\beta}}_{\lambda_{\mathrm{tgt}}}\right) \leq \frac{21 \cdot \lambda_{\mathrm{tgt}} s^{*}}{\rho_{-}-\zeta_{-}} \epsilon_{\mathrm{opt}}
$$

Therefore we conclude the proof of Theorem 4.5 in Wang et al. (2014a).

\section{D.8. Proof of Theorem 4.7 in Wang et al. (2014a).}

Proof. Recall $\widetilde{\boldsymbol{\beta}}_{t}$ is the approximate local solution obtained from the $t$-th path following stage (Lines 8 and 12 of Algorithm 1 in Wang et al. (2014a)). Therefore, it satisfies the stopping criterion of the proximal-gradient method (Line 9 of Algorithm 3 in Wang et al. (2014a)), i.e., for $t=1, \ldots, N-1$ we have $\omega_{\lambda_{t}}\left(\widetilde{\boldsymbol{\beta}}_{t}\right) \leq \lambda_{t} / 4<\lambda_{t} / 2$, while for $t=N$ we have $\omega_{\lambda_{t}}\left(\widetilde{\boldsymbol{\beta}}_{t}\right) \leq \epsilon_{\mathrm{opt}} \ll$ $\lambda_{\text {tgt }} / 4<\lambda_{t} / 2$. Meanwhile, by (5.2) in Theorem 5.5 of Wang et al. (2014a), $\widetilde{\boldsymbol{\beta}}_{t}$ satisfies $\left\|\left(\widetilde{\boldsymbol{\beta}}_{t}\right)_{S^{*}}\right\|_{0} \leq \widetilde{s}$. For logistic loss, we further have $\left\|\widetilde{\boldsymbol{\beta}}_{t}\right\|_{2} \leq R$ due to the $\ell_{2}$ constraint. Therefore Lemma 5.2 in Wang et al. (2014a) gives

$$
\left\|\widetilde{\boldsymbol{\beta}}_{t}-\boldsymbol{\beta}^{*}\right\|_{2} \leq \frac{21 / 8}{\rho_{-}-\zeta_{-}} \lambda_{t} \sqrt{s^{*}}, \quad \text { for } t=1, \ldots, N,
$$

which concludes the proof.

\section{D.9. Proof of Theorem 4.8 in Wang et al. (2014a).}

Proof. We denote the subgradients by $\boldsymbol{\xi}^{*} \in \partial\left\|\boldsymbol{\beta}^{*}\right\|_{1}$ and $\widehat{\boldsymbol{\xi}} \in \partial\left\|\widehat{\boldsymbol{\beta}}_{\lambda_{t}}\right\|_{1}$. In particular, we set $\widehat{\xi}$ to be the subgradient that attains the minimum in

$$
\omega_{\lambda_{t}}\left(\widehat{\boldsymbol{\beta}}_{\lambda_{t}}\right)=\min _{\boldsymbol{\xi}^{\prime} \in \partial\left\|\widehat{\boldsymbol{\beta}}_{\lambda_{t}}\right\|_{1}} \max _{\boldsymbol{\beta}^{\prime} \in \Omega}\left\{\frac{\left(\widehat{\boldsymbol{\beta}}_{\lambda_{t}}-\boldsymbol{\beta}^{\prime}\right)^{T}}{\left\|\widehat{\boldsymbol{\beta}}_{\lambda_{t}}-\boldsymbol{\beta}^{\prime}\right\|_{1}}\left(\nabla \widetilde{\mathcal{L}}_{\lambda_{t}}\left(\widehat{\boldsymbol{\beta}}_{\lambda_{t}}\right)+\lambda_{t} \boldsymbol{\xi}^{\prime}\right)\right\} .
$$

Recall that $\widehat{\boldsymbol{\beta}}_{\lambda_{t}}$ satisfies the exact optimality condition that $\omega_{\lambda_{t}}\left(\widehat{\boldsymbol{\beta}}_{\lambda_{t}}\right) \leq 0$, hence we have

$$
\max _{\boldsymbol{\beta}^{\prime} \in \Omega}\left\{\left(\widehat{\boldsymbol{\beta}}_{\lambda_{t}}-\boldsymbol{\beta}^{\prime}\right)^{T}\left(\nabla \widetilde{\mathcal{L}}_{\lambda_{t}}\left(\widehat{\boldsymbol{\beta}}_{\lambda_{t}}\right)+\lambda_{t} \widehat{\boldsymbol{\xi}}\right)\right\} \leq 0 .
$$

Theorem 5.5 of Wang et al. (2014a) gives $\left\|\left(\widehat{\boldsymbol{\beta}}_{\lambda_{t}}\right)_{\overline{S^{*}}}\right\|_{0} \leq \widetilde{s}$. Since

$$
\left\|\left(\widehat{\boldsymbol{\beta}}_{\lambda_{t}}-\boldsymbol{\beta}^{*}\right)_{S^{*}}\right\|_{0} \leq \widetilde{s}
$$


according to Lemma 5.1 of Wang et al. (2014a) the restricted convexity holds for $\widetilde{\mathcal{L}}_{\lambda_{t}}(\boldsymbol{\beta})$ at $\boldsymbol{\beta}_{t}$ and $\boldsymbol{\beta}^{*}$, i.e.,

$$
\begin{aligned}
& \widetilde{\mathcal{L}}_{\lambda_{t}}\left(\widehat{\boldsymbol{\beta}}_{\lambda_{t}}\right) \geq \widetilde{\mathcal{L}}_{\lambda_{t}}\left(\boldsymbol{\beta}^{*}\right)+\nabla \widetilde{\mathcal{L}}_{\lambda_{t}}\left(\boldsymbol{\beta}^{*}\right)^{T}\left(\widehat{\boldsymbol{\beta}}_{\lambda_{t}}-\boldsymbol{\beta}^{*}\right)+\frac{\rho_{-}-\zeta_{-}}{2}\left\|\widehat{\boldsymbol{\beta}}_{\lambda_{t}}-\boldsymbol{\beta}^{*}\right\|_{2}^{2} \\
& \widetilde{\mathcal{L}}_{\lambda_{t}}\left(\boldsymbol{\beta}^{*}\right) \geq \widetilde{\mathcal{L}}_{\lambda_{t}}\left(\widehat{\boldsymbol{\beta}}_{\lambda_{t}}\right)+\nabla \widetilde{\mathcal{L}}_{\lambda_{t}}\left(\widehat{\boldsymbol{\beta}}_{\lambda_{t}}\right)^{T}\left(\boldsymbol{\beta}^{*}-\widehat{\boldsymbol{\beta}}_{\lambda_{t}}\right)+\frac{\rho_{-}-\zeta_{-}}{2}\left\|\boldsymbol{\beta}^{*}-\widehat{\boldsymbol{\beta}}_{\lambda_{t}}\right\|_{2}^{2}
\end{aligned}
$$

Meanwhile, by the convexity of $\ell_{1}$ norm, we have

$$
\begin{aligned}
\lambda_{t}\left\|\widehat{\boldsymbol{\beta}}_{\lambda_{t}}\right\|_{1} & \geq \lambda_{t}\left\|\boldsymbol{\beta}^{*}\right\|_{1}+\lambda_{t}\left(\widehat{\boldsymbol{\beta}}_{\lambda_{t}}-\boldsymbol{\beta}^{*}\right)^{T} \boldsymbol{\xi}^{*}, \\
\lambda_{t}\left\|\boldsymbol{\beta}^{*}\right\|_{1} & \geq \lambda_{t}\left\|\widehat{\boldsymbol{\beta}}_{\lambda_{t}}\right\|_{1}+\lambda_{t}\left(\boldsymbol{\beta}^{*}-\widehat{\boldsymbol{\beta}}_{\lambda_{t}}\right)^{T} \widehat{\boldsymbol{\xi}} .
\end{aligned}
$$

Recall that $\widetilde{\mathcal{L}}_{\lambda}(\boldsymbol{\beta})=\mathcal{L}(\boldsymbol{\beta})+\mathcal{Q}_{\lambda}(\boldsymbol{\beta})$. Adding (D.129)-(D.132), we obtain

$$
\begin{aligned}
0 \geq \underbrace{\left(\nabla \mathcal{L}\left(\boldsymbol{\beta}^{*}\right)+\nabla \mathcal{Q}_{\lambda_{t}}\left(\boldsymbol{\beta}^{*}\right)+\lambda_{t} \boldsymbol{\xi}^{*}\right)^{T}\left(\widehat{\boldsymbol{\beta}}_{\lambda_{t}}-\boldsymbol{\beta}^{*}\right)}_{(\mathrm{i})} \\
\quad+\underbrace{\left(\nabla \widetilde{\mathcal{L}}_{\lambda_{t}}\left(\widehat{\boldsymbol{\beta}}_{\lambda_{t}}\right)+\lambda_{t} \widehat{\boldsymbol{\xi}}\right)^{T}\left(\boldsymbol{\beta}^{*}-\widehat{\boldsymbol{\beta}}_{\lambda_{t}}\right)}_{(\mathrm{ii})}+\left(\rho_{-}-\zeta_{-}\right)\left\|\widehat{\boldsymbol{\beta}}_{\lambda_{t}}-\boldsymbol{\beta}^{*}\right\|_{2}^{2} .
\end{aligned}
$$

According to (D.128) we have

$$
\left(\nabla \widetilde{\mathcal{L}}_{\lambda_{t}}\left(\widehat{\boldsymbol{\beta}}_{\lambda_{t}}\right)+\lambda_{t} \widehat{\boldsymbol{\xi}}\right)^{T}\left(\widehat{\boldsymbol{\beta}}_{\lambda_{t}}-\boldsymbol{\beta}^{*}\right) \leq \max _{\boldsymbol{\beta}^{\prime} \in \Omega}\left\{\left(\widehat{\boldsymbol{\beta}}_{\lambda_{t}}-\boldsymbol{\beta}^{\prime}\right)^{T}\left(\nabla \widetilde{\mathcal{L}}_{\lambda_{t}}\left(\widehat{\boldsymbol{\beta}}_{\lambda_{t}}\right)+\lambda \widehat{\boldsymbol{\xi}}\right)\right\} \leq 0
$$

which implies that term (ii) in (D.133) is nonnegative. Moving term (i) in (D.133) to its left-hand side, we obtain

$$
\begin{aligned}
& \left(\rho_{-}-\zeta_{-}\right)\left\|\widehat{\boldsymbol{\beta}}_{\lambda_{t}}-\boldsymbol{\beta}^{*}\right\|_{2}^{2} \\
& \leq\left(\nabla \mathcal{L}\left(\boldsymbol{\beta}^{*}\right)+\nabla \mathcal{Q}_{\lambda_{t}}\left(\boldsymbol{\beta}^{*}\right)+\lambda_{t} \boldsymbol{\xi}^{*}\right)^{T}\left(\widehat{\boldsymbol{\beta}}_{\lambda_{t}}-\boldsymbol{\beta}^{*}\right) \\
& \leq \min _{\boldsymbol{\xi}^{*} \in \partial\left\|\boldsymbol{\beta}^{*}\right\|_{1}}\left\{\sum_{j=1}^{d}\left(\left|\left(\nabla \mathcal{L}\left(\boldsymbol{\beta}^{*}\right)+\nabla \mathcal{Q}_{\lambda_{t}}\left(\boldsymbol{\beta}^{*}\right)+\lambda_{t} \boldsymbol{\xi}^{*}\right)_{j}\right| \cdot\left|\left(\boldsymbol{\beta}^{*}-\widehat{\boldsymbol{\beta}}_{\lambda_{t}}\right)_{j}\right|\right)\right\} .
\end{aligned}
$$

In the sequel, we decompose the summation on the right-hand side of (D.134) into three parts: $j \in \overline{S^{*}}, j \in S_{1}^{*}$ and $j \in S_{2}^{*}$, where $S_{1}^{*}=\left\{j:\left|\beta_{j}\right| \geq \nu_{t}\right\}$ and $S_{2}^{*}=\left\{j:\left|\beta_{j}\right|<\nu_{t}\right\}$. Here $\nu_{t}>0$ is defined in (4.16) of Wang et al. (2014a).

- For $j \in \overline{S^{*}}$, by regularity condition (c) in Wang et al. (2014a), we have

$$
\left(\nabla \mathcal{Q}_{\lambda_{t}}\left(\boldsymbol{\beta}^{*}\right)\right)_{j}=q_{\lambda_{t}}^{\prime}\left(\beta_{j}^{*}\right)=q_{\lambda_{t}}^{\prime}(0)=0, \quad \text { for } j \in \overline{S^{*}}
$$


By (4.1) in Assumption 4.1 of Wang et al. (2014a), we have

$$
\begin{aligned}
\max _{j \in \bar{S}^{*}}\left|\left(\nabla \mathcal{L}\left(\boldsymbol{\beta}^{*}\right)\right)_{j}\right| \leq \max _{1 \leq j \leq d}\left|\left(\nabla \mathcal{L}\left(\boldsymbol{\beta}^{*}\right)\right)_{j}\right| & =\left\|\nabla \mathcal{L}\left(\boldsymbol{\beta}^{*}\right)\right\|_{\infty} \\
& \leq \lambda_{\text {tgt }} / 8 \leq \lambda_{t} / 8<\lambda_{t} .
\end{aligned}
$$

Hence we have

$$
\max _{j \in \overline{S^{*}}}\left|\left(\nabla \mathcal{L}\left(\boldsymbol{\beta}^{*}\right)+\mathcal{Q}_{\lambda_{t}}\left(\boldsymbol{\beta}^{*}\right)\right)_{j}\right| \leq \lambda_{t} .
$$

Meanwhile, since $\boldsymbol{\xi}^{*} \in \partial\left\|\boldsymbol{\beta}^{*}\right\|_{1}$, we have $\lambda_{t} \boldsymbol{\xi}_{j}^{*} \in\left[-\lambda_{t}, \lambda_{t}\right]$. Therefore, for any $j \in \overline{S^{*}}$, we can always find a $\xi_{j}^{*}$ such that

$$
\left|\left(\nabla \mathcal{L}\left(\boldsymbol{\beta}^{*}\right)+\nabla \mathcal{Q}_{\lambda_{t}}\left(\boldsymbol{\beta}^{*}\right)\right)_{j}+\lambda_{t} \xi_{j}^{*}\right|=0,
$$

which implies

$$
\min _{\boldsymbol{\xi}^{*} \in \partial\left\|\boldsymbol{\beta}^{*}\right\|_{1}}\left\{\left|\left(\nabla \mathcal{L}\left(\boldsymbol{\beta}^{*}\right)+\nabla \mathcal{Q}_{\lambda_{t}}\left(\boldsymbol{\beta}^{*}\right)+\lambda_{t} \boldsymbol{\xi}^{*}\right)_{j}\right|\right\}=0, \quad \text { for } j \in \overline{S^{*}} .
$$

Thus we obtain

$$
\min _{\boldsymbol{\xi}^{*} \in \partial\left\|\boldsymbol{\beta}^{*}\right\|_{1}}\left\{\sum_{j \in \overline{S^{*}}}\left|\left(\nabla \mathcal{L}\left(\boldsymbol{\beta}^{*}\right)+\nabla \mathcal{Q}_{\lambda_{t}}\left(\boldsymbol{\beta}^{*}\right)+\lambda_{t} \boldsymbol{\xi}^{*}\right)_{j}\right| \cdot\left|\left(\boldsymbol{\beta}^{*}-\widehat{\boldsymbol{\beta}}_{\lambda_{t}}\right)_{j}\right|\right\}=0 .
$$

- For $j \in S_{1}^{*} \subseteq S^{*}$, we have $\left|\beta_{j}^{*}\right| \geq \nu_{t}$. Recall that $\mathcal{P}_{\lambda}(\boldsymbol{\beta})=\mathcal{Q}_{\lambda}(\boldsymbol{\beta})+\lambda\|\boldsymbol{\beta}\|_{1}$. By our assumption on $\mathcal{P}_{\lambda_{t}}(\boldsymbol{\beta})$ in (4.16) of Wang et al. (2014a), we have

$$
\left(\nabla \mathcal{Q}_{\lambda_{t}}\left(\boldsymbol{\beta}^{*}\right)+\lambda_{t} \boldsymbol{\xi}^{*}\right)_{j}=p_{\lambda_{t}}^{\prime}\left(\beta_{j}^{*}\right)=0, \quad \text { for } j \in S_{1}^{*},
$$

which implies

$$
\begin{aligned}
& \min _{\boldsymbol{\xi}^{*} \in \partial\left\|\boldsymbol{\beta}^{*}\right\|_{1}}\left\{\sum_{j \in S_{1}^{*}}\left(\left|\left(\nabla \mathcal{L}\left(\boldsymbol{\beta}^{*}\right)+\nabla \mathcal{Q}_{\lambda_{t}}\left(\boldsymbol{\beta}^{*}\right)+\lambda_{t} \boldsymbol{\xi}^{*}\right)_{j}\right| \cdot\left|\left(\boldsymbol{\beta}^{*}-\widehat{\boldsymbol{\beta}}_{\lambda_{t}}\right)_{j}\right|\right)\right\} \\
& =\sum_{j \in S_{1}^{*}}\left|\left(\nabla \mathcal{L}\left(\boldsymbol{\beta}^{*}\right)\right)_{j}\right| \cdot\left|\left(\boldsymbol{\beta}^{*}-\widehat{\boldsymbol{\beta}}_{\lambda_{t}}\right)_{j}\right| \\
& \leq\left\|\left(\nabla \mathcal{L}\left(\boldsymbol{\beta}^{*}\right)\right)_{S_{1}^{*}}\right\|_{2} \cdot\left\|\boldsymbol{\beta}^{*}-\widehat{\boldsymbol{\beta}}_{\lambda_{t}}\right\|_{2} .
\end{aligned}
$$

- For $j \in S_{2}^{*} \subseteq S^{*}$, we have $\left|\beta_{j}^{*}\right|<\nu_{t}$. According to (4.1) in Assumption 4.1 of Wang et al. (2014a), we have

$$
\max _{j \in S_{2}^{*}}\left|\left(\nabla \mathcal{L}\left(\boldsymbol{\beta}^{*}\right)\right)_{j}\right| \leq \max _{1 \leq j \leq d}\left|\left(\nabla \mathcal{L}\left(\boldsymbol{\beta}^{*}\right)\right)_{j}\right|=\left\|\nabla \mathcal{L}\left(\boldsymbol{\beta}^{*}\right)\right\|_{\infty} \leq \lambda_{t} / 8 \leq \lambda_{t} / 8 .
$$


Meanwhile we have

$$
\max _{j \in S_{2}^{*}}\left|\left(\nabla \mathcal{Q}_{\lambda_{t}}\left(\boldsymbol{\beta}^{*}\right)\right)_{j}\right|=\max _{j \in S_{2}^{*}}\left|q_{\lambda_{t}}^{\prime}\left(\beta_{j}^{*}\right)\right| \leq \max _{1 \leq j \leq d}\left|q_{\lambda_{t}}^{\prime}\left(\beta_{j}^{*}\right)\right| \leq \lambda_{t},
$$

where the last inequality follows from regularity condition (d) in Wang et al. (2014a). Also, since $\boldsymbol{\xi}^{*} \in \partial\left\|\boldsymbol{\beta}^{*}\right\|_{1}$, we have $\left|\xi_{j}^{*}\right| \leq 1$. Therefore we obtain that, for $j \in S_{2}^{*}$,

$$
\begin{aligned}
& \left|\left(\nabla \mathcal{L}\left(\boldsymbol{\beta}^{*}\right)+\nabla \mathcal{Q}_{\lambda_{t}}\left(\boldsymbol{\beta}^{*}\right)+\lambda_{t} \boldsymbol{\xi}^{*}\right)_{j}\right| \\
& \quad \leq \max _{j \in S_{2}^{*}}\left|\left(\nabla \mathcal{L}\left(\boldsymbol{\beta}^{*}\right)\right)_{j}\right|+\max _{j \in S_{2}^{*}}\left|\left(\nabla \mathcal{Q}_{\lambda_{t}}\left(\boldsymbol{\beta}^{*}\right)\right)_{j}\right|+\lambda_{t} \leq 3 \lambda_{t} .
\end{aligned}
$$

which implies

(D.137)

$$
\begin{aligned}
& \min _{\boldsymbol{\xi}^{*} \in \partial\left\|\boldsymbol{\beta}^{*}\right\|_{1}}\left\{\sum_{j \in S_{2}^{*}}\left|\left(\nabla \mathcal{L}\left(\boldsymbol{\beta}^{*}\right)+\nabla \mathcal{Q}_{\lambda_{t}}\left(\boldsymbol{\beta}^{*}\right)+\lambda_{t} \boldsymbol{\xi}^{*}\right)_{j}\right| \cdot\left|\left(\boldsymbol{\beta}^{*}-\widehat{\boldsymbol{\beta}}_{\lambda_{t}}\right)_{j}\right|\right\} \\
& \leq 3 \lambda_{t} \sum_{j \in S_{2}^{*}}\left|\left(\boldsymbol{\beta}^{*}-\widehat{\boldsymbol{\beta}}_{\lambda_{t}}\right)_{j}\right| \\
& =3 \lambda_{t}\left\|\left(\boldsymbol{\beta}^{*}-\widehat{\boldsymbol{\beta}}_{\lambda_{t}}\right)_{\overline{S_{2}^{*}}}\right\|_{1} \\
& \leq 3 \lambda_{t} \sqrt{s^{*}}\left\|\left(\boldsymbol{\beta}^{*}-\widehat{\boldsymbol{\beta}}_{\lambda_{t}}\right)_{\overline{S_{2}^{*}}}\right\|_{2} \\
& \leq 3 \lambda_{t} \sqrt{s_{2}^{*}}\left\|\boldsymbol{\beta}^{*}-\widehat{\boldsymbol{\beta}}_{\lambda_{t}}\right\|_{2} .
\end{aligned}
$$

Plugging (D.135)-(D.137) into the right-hand side of (D.134), we obtain

$$
\left\|\widehat{\boldsymbol{\beta}}_{\lambda_{t}}-\boldsymbol{\beta}^{*}\right\|_{2} \leq \frac{1}{\rho_{-}-\zeta_{-}}\left(\left\|\left(\nabla \mathcal{L}\left(\boldsymbol{\beta}^{*}\right)\right)_{S_{1}^{*}}\right\|_{2}+3 \lambda_{t} \sqrt{s_{2}^{*}}\right)
$$

which concludes the proof of Theorem 4.8 in Wang et al. (2014a).

D.10. Proof for Lemma 4.9 and Theorem 4.10. First, we prove Lemma 4.9 in Wang et al. (2014a), which states that the oracle estimator $\widehat{\boldsymbol{\beta}}_{\mathrm{O}}$ is uniquely defined and has nice statistical recovery property.

Proof. To prove that the global minimizer of (4.19) in Wang et al. (2014a) is unique even for nonconvex loss functions, in the following we show that $\mathcal{L}(\boldsymbol{\beta})$ is strongly convex on the sparse set $\left\{\boldsymbol{\beta}: \operatorname{supp}(\boldsymbol{\beta}) \subseteq S^{*}\right\}$. We assume that $\boldsymbol{\beta}$ and $\boldsymbol{\beta}^{\prime}$ satisfy $\operatorname{supp}(\boldsymbol{\beta}) \subseteq S^{*}$ and $\operatorname{supp}\left(\boldsymbol{\beta}^{\prime}\right) \subseteq S^{*}$. By Taylor's theorem and the mean value theorem, we have

$$
\mathcal{L}\left(\boldsymbol{\beta}^{\prime}\right)=\mathcal{L}(\boldsymbol{\beta})+\nabla \mathcal{L}(\boldsymbol{\beta})^{T}\left(\boldsymbol{\beta}^{\prime}-\boldsymbol{\beta}\right)+\frac{1}{2}\left(\boldsymbol{\beta}^{\prime}-\boldsymbol{\beta}\right)^{T} \nabla^{2} \mathcal{L}\left(\gamma \boldsymbol{\beta}^{\prime}+(1-\gamma) \boldsymbol{\beta}\right)\left(\boldsymbol{\beta}^{\prime}-\boldsymbol{\beta}\right)
$$


where $\gamma \in[0,1]$. Note that we have $\left\|\boldsymbol{\beta}^{\prime}-\boldsymbol{\beta}\right\|_{0}=s^{*}<s^{*}+2 \widetilde{s}$. By Definition 4.2 and Definition 4.3 in Wang et al. (2014a), we have

$$
\frac{\left(\boldsymbol{\beta}^{\prime}-\boldsymbol{\beta}\right)^{T}}{\left\|\boldsymbol{\beta}^{\prime}-\boldsymbol{\beta}\right\|_{2}} \nabla^{2} \mathcal{L}\left(\gamma \boldsymbol{\beta}+(1-\gamma) \boldsymbol{\beta}^{\prime}\right) \frac{\left(\boldsymbol{\beta}^{\prime}-\boldsymbol{\beta}\right)}{\left\|\boldsymbol{\beta}^{\prime}-\boldsymbol{\beta}\right\|_{2}} \geq \rho_{-}\left(\nabla^{2} \mathcal{L}, s^{*}+2 \widetilde{s}\right) .
$$

Plugging this into the right-hand side of (D.138), we obtain

$$
\mathcal{L}\left(\boldsymbol{\beta}^{\prime}\right) \geq \mathcal{L}(\boldsymbol{\beta})+\nabla \mathcal{L}(\boldsymbol{\beta})^{T}\left(\boldsymbol{\beta}^{\prime}-\boldsymbol{\beta}\right)+\frac{\rho_{-}}{2}\left\|\boldsymbol{\beta}^{\prime}-\boldsymbol{\beta}\right\|_{2}^{2},
$$

where $\rho_{-}=\rho_{-}\left(\nabla^{2} \mathcal{L}, s^{*}+2 \widetilde{s}\right)$ is a positive constant according to Assumption 4.4. Note that (D.139) holds for any $\boldsymbol{\beta}$ and $\boldsymbol{\beta}^{\prime}$ such that $\operatorname{supp}(\boldsymbol{\beta}) \subseteq S^{*}$ and $\operatorname{supp}\left(\boldsymbol{\beta}^{\prime}\right) \subseteq S^{*}$. Therefore, $\mathcal{L}(\boldsymbol{\beta})$ is strongly convex on this sparse set, which implies the minimizer of (4.19) in Wang et al. (2014a) is unique.

Now we prove the statistical recovery property of the oracle estimator $\widehat{\boldsymbol{\beta}}_{\mathrm{O}}$ in the setting where $\mathcal{L}(\boldsymbol{\beta})$ is least squares loss. Let $\widehat{\boldsymbol{\beta}}_{\mathrm{O}}^{\prime}, \boldsymbol{\beta}^{* \prime} \in \mathbb{R}^{s^{*}}$ be the restrictions of $\widehat{\boldsymbol{\beta}}_{\mathrm{O}}, \boldsymbol{\beta}^{*} \in \mathbb{R}^{d}$ to $S^{*}$ respectively, and $\mathbf{X}_{S^{*}} \in \mathbb{R}^{n \times s^{*}}$ be a new matrix containing the columns of $\mathbf{X}$, i.e., $\mathbf{X}_{j}$, that satisfy $j \in S^{*}$. Since $\widehat{\boldsymbol{\beta}}_{\mathrm{O}}^{\prime}$ is the solution to the ordinary least squares problem

$$
\widehat{\boldsymbol{\beta}}_{\mathrm{O}}^{\prime}=\underset{\boldsymbol{\beta}^{\prime} \in \mathbb{R}^{s^{*}}}{\operatorname{argmin}} \frac{1}{2 n}\left\|\mathbf{X}_{S^{*}} \boldsymbol{\beta}^{\prime}-\mathbf{y}\right\|_{2}^{2}
$$

it has the closed-form expression of

$$
\widehat{\boldsymbol{\beta}}_{\mathrm{O}}^{\prime}=\left(\mathbf{X}_{S^{*}}^{T} \mathbf{X}_{S^{*}}\right)^{-1} \mathbf{X}_{S^{*}}^{T} \mathbf{y}
$$

Here we still need to prove that $\mathbf{X}_{S^{*}}^{T} \mathbf{X}_{S^{*}} \in \mathbb{R}^{s^{*} \times s^{*}}$ is invertible. Note that the smallest eigenvalue of $\mathbf{X}_{S^{*}}^{T} \mathbf{X}_{S^{*}}$ is defined as

$$
\Lambda_{\min }\left(\mathbf{X}_{S^{*}}^{T} \mathbf{X}_{S^{*}}\right)=\inf \left\{\boldsymbol{v}^{T} \mathbf{X}_{S^{*}}^{T} \mathbf{X}_{S^{*}} \boldsymbol{v}:\|\boldsymbol{v}\|_{2}=1, \boldsymbol{v} \in \mathbb{R}^{s^{*}}\right\}
$$

which satisfies

$$
\begin{aligned}
\Lambda_{\min }\left(\mathbf{X}_{S^{*}}^{T} \mathbf{X}_{S^{*}}\right) & =\inf \left\{\boldsymbol{v}^{T} \mathbf{X}^{T} \mathbf{X} \boldsymbol{v}:\|\boldsymbol{v}\|_{2}=1, \boldsymbol{v} \in \mathbb{R}^{d}, \operatorname{supp}(\boldsymbol{v})=S^{*}\right\} \\
& \geq \inf \left\{\boldsymbol{v}^{T} \mathbf{X}^{T} \mathbf{X} \boldsymbol{v}:\|\boldsymbol{v}\|_{2}=1, \boldsymbol{v} \in \mathbb{R}^{d},\|\boldsymbol{v}\|_{0} \leq s^{*}\right\} \\
& \geq \inf \left\{\boldsymbol{v}^{T} \mathbf{X}^{T} \mathbf{X} \boldsymbol{v}:\|\boldsymbol{v}\|_{2}=1, \boldsymbol{v} \in \mathbb{R}^{d},\|\boldsymbol{v}\|_{0} \leq s^{*}+2 \widetilde{s}\right\} \\
& =n \rho_{-}\left(\nabla^{2} \mathcal{L}, s^{*}+2 \widetilde{s}\right) \\
& >0 .
\end{aligned}
$$

Here the first and second inequality are due to $\left\{\boldsymbol{v}: \operatorname{supp}(\boldsymbol{v})=S^{*}\right\} \subseteq\{\boldsymbol{v}$ : $\left.\|\boldsymbol{v}\|_{0} \leq s^{*}\right\} \subseteq\left\{\boldsymbol{v}:\|\boldsymbol{v}\|_{0} \leq s^{*}+2 \widetilde{s}\right\}$, while the second equality follows from Definition 4.2 in Wang et al. (2014a), since for least squares loss $\nabla^{2} \mathcal{L}(\boldsymbol{\beta})=\mathbf{X}^{T} \mathbf{X} / n$, and the last inequality follows from Assumption 4.4 in Wang et al. (2014a). Therefore the smallest eigenvalue of $\mathbf{X}_{S^{*}}^{T} \mathbf{X}_{S^{*}}$ is positive, 
which implies that $\mathbf{X}_{S^{*}}^{T} \mathbf{X}_{S^{*}}$ is invertible.

By our assumption on $\left(Y \mid \boldsymbol{X}=\mathbf{x}_{i}\right)$, we have $\mathbf{y}=\mathbf{X} \boldsymbol{\beta}^{*}+\boldsymbol{\epsilon}=\mathbf{X}_{S^{*}} \boldsymbol{\beta}^{* \prime}+\boldsymbol{\epsilon}$, where $\epsilon \in \mathbb{R}^{n}$ is a zero mean sub-Gaussian random vector with independent entries and variance proxy $\sigma^{2}$. Therefore, we have

$$
\begin{aligned}
\widehat{\boldsymbol{\beta}}_{\mathrm{O}}^{\prime}-\boldsymbol{\beta}^{* \prime}=\left(\mathbf{X}_{S^{*}}^{T} \mathbf{X}_{S^{*}}\right)^{-1} \mathbf{X}_{S^{*}}^{T} \mathbf{y}-\boldsymbol{\beta}^{* \prime} & =\left(\mathbf{X}_{S^{*}}^{T} \mathbf{X}_{S^{*}}\right)^{-1} \mathbf{X}_{S^{*}}^{T}\left(\mathbf{X} \boldsymbol{\beta}^{*}+\boldsymbol{\epsilon}\right)-\boldsymbol{\beta}^{* \prime} \\
& =\left(\mathbf{X}_{S^{*}}^{T} \mathbf{X}_{S^{*}}\right)^{-1} \mathbf{X}_{S^{*}}^{T} \boldsymbol{\epsilon} .
\end{aligned}
$$

Now we provide an upper bound of $\left\|\widehat{\boldsymbol{\beta}}_{\mathrm{O}}^{\prime}-\boldsymbol{\beta}^{* \prime}\right\|_{\infty}$. Note that the $j$-th entry of $\left(\mathbf{X}_{S^{*}}^{T} \mathbf{X}_{S^{*}}\right)^{-1} \mathbf{X}_{S^{*}}^{T} \boldsymbol{\epsilon} \in \mathbb{R}^{s^{*}}$ could be denoted as $\boldsymbol{e}_{j}\left(\mathbf{X}_{S^{*}}^{T} \mathbf{X}_{S^{*}}\right)^{-1} \mathbf{X}_{S^{*}}^{T} \boldsymbol{\epsilon}$. Here $\boldsymbol{e}_{j} \in \mathbb{R}^{s^{*}}$ denotes a vector that is all-zero expect an "1" in its $j$-th coordinate. Hence, for any $j, \boldsymbol{e}_{j}\left(\mathbf{X}_{S^{*}}^{T} \mathbf{X}_{S^{*}}\right)^{-1} \mathbf{X}_{S^{*}}^{T} \boldsymbol{\epsilon}$ is sub-Gaussian with variance proxy $\left\|\boldsymbol{e}_{j}\left(\mathbf{X}_{S^{*}}^{T} \mathbf{X}_{S^{*}}\right)^{-1} \mathbf{X}_{S^{*}}^{T}\right\|_{2}^{2} \sigma^{2}$. Therefore we have

$$
\mathbb{P}\left(\left|\boldsymbol{e}_{j}\left(\mathbf{X}_{S^{*}}^{T} \mathbf{X}_{S^{*}}\right)^{-1} \mathbf{X}_{S^{*}}^{T} \boldsymbol{\epsilon}\right|>t\right) \leq 2 \exp \left(-t^{2} /\left(\left\|\boldsymbol{e}_{j}\left(\mathbf{X}_{S^{*}}^{T} \mathbf{X}_{S^{*}}\right)^{-1} \mathbf{X}_{S^{*}}^{T}\right\|_{2}^{2} \sigma^{2}\right)\right),
$$

which implies

$$
\begin{aligned}
& \mathbb{P}\left(\max _{j \in\left\{1, \ldots, s^{*}\right\}}\left|\boldsymbol{e}_{j}\left(\mathbf{X}_{S^{*}}^{T} \mathbf{X}_{S^{*}}\right)^{-1} \mathbf{X}_{S^{*}}^{T} \boldsymbol{\epsilon}\right|>t\right) \\
& \quad \leq 2 s^{*} \exp \left(-t^{2} /\left(\max _{j \in\left\{1, \ldots, s^{*}\right\}}\left\|\boldsymbol{e}_{j}\left(\mathbf{X}_{S^{*}}^{T} \mathbf{X}_{S^{*}}\right)^{-1} \mathbf{X}_{S^{*}}^{T}\right\|_{2}^{2} \sigma^{2}\right)\right)
\end{aligned}
$$

Taking $t=C \max _{j \in\left\{1, \ldots, s^{*}\right\}}\left\|\boldsymbol{e}_{j}\left(\mathbf{X}_{S^{*}}^{T} \mathbf{X}_{S^{*}}\right)^{-1} \mathbf{X}_{S^{*}}^{T}\right\|_{2} \sigma \cdot \sqrt{2 \log s^{*}}$ with $C>0$, we have that

$$
\begin{aligned}
\left\|\widehat{\boldsymbol{\beta}}_{\mathrm{O}}^{\prime}-\boldsymbol{\beta}^{* \prime}\right\|_{\infty} & =\left\|\left(\mathbf{X}_{S^{*}}^{T} \mathbf{X}_{S^{*}}\right)^{-1} \mathbf{X}_{S^{*}}^{T} \boldsymbol{\epsilon}\right\|_{\infty} \\
& =\max _{j \in\left\{1, \ldots, s^{*}\right\}}\left|\boldsymbol{e}_{j}\left(\mathbf{X}_{S^{*}}^{T} \mathbf{X}_{S^{*}}\right)^{-1} \mathbf{X}_{S^{*}}^{T} \boldsymbol{\epsilon}\right| \\
& \leq C \max _{j \in\left\{1, \ldots, s^{*}\right\}}\left\|\boldsymbol{e}_{j}\left(\mathbf{X}_{S^{*}}^{T} \mathbf{X}_{S^{*}}\right)^{-1} \mathbf{X}_{S^{*}}^{T}\right\|_{2} \sigma \cdot \sqrt{2 \log s^{*}}
\end{aligned}
$$

holds with probability at least $1-2 \exp \left(-C^{2}\right) / s^{*}$. In other words, there exists a constant $C>0$ sufficiently large such that (D.141) holds with high probability. Note that, for any $j \in\{1, \ldots, d\}$

$$
\begin{aligned}
\left\|\boldsymbol{e}_{j}\left(\mathbf{X}_{S^{*}}^{T} \mathbf{X}_{S^{*}}\right)^{-1} \mathbf{X}_{S^{*}}^{T}\right\|_{2}^{2} & =\boldsymbol{e}_{j}\left(\mathbf{X}_{S^{*}}^{T} \mathbf{X}_{S^{*}}\right)^{-1} \mathbf{X}_{S^{*}}^{T} \mathbf{X}_{S^{*}}\left(\mathbf{X}_{S^{*}}^{T} \mathbf{X}_{S^{*}}\right)^{-1} \boldsymbol{e}_{j}^{T} \\
& =\boldsymbol{e}_{j}\left(\mathbf{X}_{S^{*}}^{T} \mathbf{X}_{S^{*}}\right)^{-1} \boldsymbol{e}_{j}^{T} \\
& \leq \Lambda_{\max }\left(\left(\mathbf{X}_{S^{*}}^{T} \mathbf{X}_{S^{*}}\right)^{-1}\right) \\
& =1 / \Lambda_{\min }\left(\mathbf{X}_{S^{*}}^{T} \mathbf{X}_{S^{*}}\right) \\
& \leq 1 /\left(n \rho_{-}\right)
\end{aligned}
$$

where the last inequality follows from (D.140). Plugging this into (D.141), 
we obtain

$$
\left\|\widehat{\boldsymbol{\beta}}_{\mathrm{O}}^{\prime}-\boldsymbol{\beta}^{* \prime}\right\|_{\infty} \leq C \sigma \sqrt{2 / \rho_{-}} \cdot \sqrt{\frac{\log s^{*}}{n}} .
$$

We remind that $\widehat{\boldsymbol{\beta}}_{\mathrm{O}}^{\prime}$ and $\boldsymbol{\beta}^{* \prime}$ are the restrictions of $\widehat{\boldsymbol{\beta}}_{\mathrm{O}}$ and $\boldsymbol{\beta}^{*}$ to $S^{*}$, and $\operatorname{supp}\left(\widehat{\boldsymbol{\beta}}_{\mathrm{O}}\right) \subseteq S^{*}$. Therefore we obtain

$$
\left\|\widehat{\boldsymbol{\beta}}_{\mathrm{O}}-\boldsymbol{\beta}^{*}\right\|_{\infty} \leq C \sigma \sqrt{2 / \rho_{-}} \cdot \sqrt{\frac{\log s^{*}}{n}},
$$

which concludes the proof.

Now we prove Theorem 4.10 in Wang et al. (2014a).

Proof. Let $\widehat{\boldsymbol{\xi}} \in \partial\left\|\widehat{\boldsymbol{\beta}}_{\lambda_{t}}\right\|_{1}$. We set $\widehat{\boldsymbol{\xi}}$ as the subgradient which attains the minimum in

$$
\omega_{\lambda_{t}}\left(\widehat{\boldsymbol{\beta}}_{\lambda_{t}}\right)=\min _{\boldsymbol{\xi}^{\prime} \in \partial\left\|\widehat{\boldsymbol{\beta}}_{\lambda_{t}}\right\|_{1}} \max _{\boldsymbol{\beta}^{\prime} \in \Omega}\left\{\frac{\left(\widehat{\boldsymbol{\beta}}_{\lambda_{t}}-\boldsymbol{\beta}^{\prime}\right)^{T}}{\left\|\widehat{\boldsymbol{\beta}}_{\lambda_{t}}-\boldsymbol{\beta}^{\prime}\right\|_{1}}\left(\nabla \widetilde{\mathcal{L}}_{\lambda_{t}}\left(\widehat{\boldsymbol{\beta}}_{\lambda_{t}}\right)+\lambda_{t} \boldsymbol{\xi}^{\prime}\right)\right\} .
$$

Since $\widehat{\boldsymbol{\beta}}_{\lambda_{t}}$ satisfies the exact optimality condition that $\omega_{\lambda_{t}}\left(\widehat{\boldsymbol{\beta}}_{\lambda_{t}}\right) \leq 0$, we have

$$
\max _{\boldsymbol{\beta}^{\prime} \in \Omega}\left\{\left(\widehat{\boldsymbol{\beta}}_{\lambda_{t}}-\boldsymbol{\beta}^{\prime}\right)^{T}\left(\nabla \widetilde{\mathcal{L}}_{\lambda_{t}}\left(\widehat{\boldsymbol{\beta}}_{\lambda_{t}}\right)+\lambda_{t} \widehat{\boldsymbol{\xi}}\right)\right\} \leq 0 .
$$

Now we prove that there exists some $\boldsymbol{\xi}_{\mathrm{O}} \in \partial\left\|\widehat{\boldsymbol{\beta}}_{\mathrm{O}}\right\|_{1}$, such that $\widehat{\boldsymbol{\beta}}_{\mathrm{O}}$ satisfies the same exact optimality condition

$$
\max _{\boldsymbol{\beta}^{\prime} \in \Omega}\left\{\left(\widehat{\boldsymbol{\beta}}_{\mathrm{O}}-\boldsymbol{\beta}^{\prime}\right)^{T}\left(\nabla \widetilde{\mathcal{L}}_{\lambda_{t}}\left(\widehat{\boldsymbol{\beta}}_{\mathrm{O}}\right)+\lambda_{t} \boldsymbol{\xi}_{\mathrm{O}}\right)\right\} \leq 0 .
$$

Recall that $\widetilde{\mathcal{L}}_{\lambda}(\boldsymbol{\beta})=\mathcal{L}(\boldsymbol{\beta})+\mathcal{Q}_{\lambda}(\boldsymbol{\beta})$. In (D.143), we have

$$
\begin{aligned}
& \left(\widehat{\boldsymbol{\beta}}_{\mathrm{O}}-\boldsymbol{\beta}^{\prime}\right)^{T}\left(\nabla \widetilde{\mathcal{L}}_{\lambda_{t}}\left(\widehat{\boldsymbol{\beta}}_{\mathrm{O}}\right)+\lambda_{t} \boldsymbol{\xi}_{\mathrm{O}}\right) \\
& =\underbrace{\sum_{j \in S^{*}}\left(\widehat{\boldsymbol{\beta}}_{\mathrm{O}}-\boldsymbol{\beta}^{\prime}\right)_{j}\left(\nabla \widetilde{\mathcal{L}}_{\lambda_{t}}\left(\widehat{\boldsymbol{\beta}}_{\mathrm{O}}\right)+\lambda_{t} \boldsymbol{\xi}_{\mathrm{O}}\right)_{j}}_{(\mathrm{i})}+\underbrace{\sum_{j \in \bar{S}^{*}}\left(\widehat{\boldsymbol{\beta}}_{\mathrm{O}}-\boldsymbol{\beta}^{\prime}\right)_{j}\left(\nabla \widetilde{\mathcal{L}}_{\lambda_{t}}\left(\widehat{\boldsymbol{\beta}}_{\mathrm{O}}\right)+\lambda_{t} \boldsymbol{\xi}_{\mathrm{O}}\right)_{j}}_{(\mathrm{ii})} .
\end{aligned}
$$

For term (i) in (D.144), according to Lemma 4.9 of Wang et al. (2014a) we have, for $n$ sufficiently large,

$$
\left|\left(\widehat{\boldsymbol{\beta}}_{\mathrm{O}}\right)_{j}\right| \geq\left|\beta_{j}^{*}\right|-\left\|\widehat{\boldsymbol{\beta}}_{\mathrm{O}}-\boldsymbol{\beta}^{*}\right\|_{\infty} \geq 2 \nu_{t}-\sigma \sqrt{2 / \rho_{-}} \cdot \sqrt{\frac{\log s^{*}}{n}} \geq \nu_{t} .
$$


Recall that $\mathcal{P}_{\lambda}(\boldsymbol{\beta})=\mathcal{Q}_{\lambda}(\boldsymbol{\beta})+\lambda\|\boldsymbol{\beta}\|_{1}$. Hence we have

$$
\left(\nabla \mathcal{Q}_{\lambda_{t}}\left(\widehat{\boldsymbol{\beta}}_{\mathrm{O}}\right)+\lambda_{t} \boldsymbol{\xi}_{\mathrm{O}}\right)_{j}=\left(\nabla \mathcal{P}_{\lambda_{t}}\left(\widehat{\boldsymbol{\beta}}_{\mathrm{O}}\right)\right)_{j}=p_{\lambda_{t}}^{\prime}\left(\left(\widehat{\boldsymbol{\beta}}_{\mathrm{O}}\right)_{j}\right)=0
$$

where the last equality is from (4.16) of Wang et al. (2014a). Then we have

$$
\begin{aligned}
& \sum_{j \in S^{*}}\left(\widehat{\boldsymbol{\beta}}_{\mathrm{O}}-\boldsymbol{\beta}^{\prime}\right)_{j}\left(\nabla \widetilde{\mathcal{L}}_{\lambda_{t}}\left(\widehat{\boldsymbol{\beta}}_{\mathrm{O}}\right)+\lambda_{t} \boldsymbol{\xi}_{\mathrm{O}}\right)_{j} \\
& =\sum_{j \in S^{*}}\left(\widehat{\boldsymbol{\beta}}_{\mathrm{O}}-\boldsymbol{\beta}^{\prime}\right)_{j}\left(\nabla \mathcal{L}\left(\widehat{\boldsymbol{\beta}}_{\mathrm{O}}\right)+\nabla \mathcal{Q}_{\lambda_{t}}\left(\widehat{\boldsymbol{\beta}}_{\mathrm{O}}\right)+\lambda_{t} \boldsymbol{\xi}_{\mathrm{O}}\right)_{j} \\
& =\sum_{j \in S^{*}}\left(\widehat{\boldsymbol{\beta}}_{\mathrm{O}}-\boldsymbol{\beta}^{\prime}\right)_{j}\left(\nabla \mathcal{L}\left(\widehat{\boldsymbol{\beta}}_{\mathrm{O}}\right)\right)_{j},
\end{aligned}
$$

where the first equality follows from $\widetilde{\mathcal{L}}_{\lambda}(\boldsymbol{\beta})=\mathcal{L}(\boldsymbol{\beta})+\mathcal{Q}_{\lambda}(\boldsymbol{\beta})$, and the second follows from (D.146). We remind that $\widehat{\boldsymbol{\beta}}_{\mathrm{O}}$ is the global solution to the oracle minimization problem in (4.19) of Wang et al. (2014a). Thus $\widehat{\boldsymbol{\beta}}_{\mathrm{O}}$ satisfies the exact optimality condition of (4.19)

$$
\max _{\boldsymbol{\beta}^{\prime} \in \Omega}\left\{\sum_{j \in S^{*}}\left(\widehat{\boldsymbol{\beta}}_{\mathrm{O}}-\boldsymbol{\beta}^{\prime}\right)_{j}\left(\nabla \mathcal{L}\left(\widehat{\boldsymbol{\beta}}_{\mathrm{O}}\right)\right)_{j}\right\} \leq 0 .
$$

Thus, taking maximum over $\boldsymbol{\beta}^{\prime} \in \Omega$ on both sides of (D.147), we have that, the maximum of term (i) over $\boldsymbol{\beta}^{\prime} \in \Omega$ is upper bounded by zero.

For term (ii) in (D.144), remind that $\left(\widehat{\boldsymbol{\beta}}_{\mathrm{O}}\right)_{j}=0$ for $j \in \overline{S^{*}}$. According to regularity condition (c) we have

$$
\left(\nabla \mathcal{Q}_{\lambda_{t}}\left(\widehat{\boldsymbol{\beta}}_{\mathrm{O}}\right)\right)_{j}=0
$$

Meanwhile, for any $j \in \overline{S^{*}}$ we have

$$
\left|\left(\nabla \mathcal{L}\left(\widehat{\boldsymbol{\beta}}_{\mathrm{O}}\right)\right)_{j}\right|=\left|\left(\nabla \mathcal{L}\left(\boldsymbol{\beta}^{*}\right)\right)_{j}\right| \leq\left\|\nabla \mathcal{L}\left(\boldsymbol{\beta}^{*}\right)\right\|_{\infty} \leq \lambda_{\text {tgt }} / 8
$$

where the first equality is because $\boldsymbol{\beta}_{j}^{*}=\left(\widehat{\boldsymbol{\beta}}_{\mathrm{O}}\right)_{j}=0$, while the last inequality follows from Assumption 4.1 of Wang et al. (2014a). Therefore, since $\boldsymbol{\xi}_{\mathrm{O}} \in$ $\partial\left\|\widehat{\boldsymbol{\beta}}_{\mathrm{O}}\right\|_{1}$, for

$$
\left(\nabla \widetilde{\mathcal{L}}_{\lambda_{t}}\left(\widehat{\boldsymbol{\beta}}_{\mathrm{O}}\right)+\lambda_{t} \boldsymbol{\xi}_{\mathrm{O}}\right)_{j}=\left(\nabla \mathcal{L}\left(\widehat{\boldsymbol{\beta}}_{\mathrm{O}}\right)+\nabla \mathcal{Q}_{\lambda_{t}}\left(\widehat{\boldsymbol{\beta}}_{\mathrm{O}}\right)+\lambda_{t} \boldsymbol{\xi}_{\mathrm{O}}\right)_{j}=\left(\nabla \mathcal{L}\left(\widehat{\boldsymbol{\beta}}_{\mathrm{O}}\right)+\lambda_{t} \boldsymbol{\xi}_{\mathrm{O}}\right)_{j},
$$

we can set $\left(\boldsymbol{\xi}_{\mathrm{O}}\right)_{j}=-\left(\nabla \mathcal{L}\left(\widehat{\boldsymbol{\beta}}_{\mathrm{O}}\right) / \lambda_{t}\right)_{j}$. Then we obtain, for any $j \in \overline{S^{*}}$,

$$
\left(\nabla \widetilde{\mathcal{L}}_{\lambda_{t}}\left(\widehat{\boldsymbol{\beta}}_{\mathrm{O}}\right)+\lambda_{t} \boldsymbol{\xi}_{\mathrm{O}}\right)_{j}=0
$$

which further implies that term (ii) in (D.144) is zero.

In summary, taking maximum over $\boldsymbol{\beta}^{\prime} \in \Omega$ on both sides of (D.144), we 
obtain (D.143).

Now we are ready to prove that $\widehat{\boldsymbol{\beta}}_{\lambda_{t}}=\widehat{\boldsymbol{\beta}}_{\mathrm{O}}$. Recall that the oracle estimator satisfies $\operatorname{supp}\left(\widehat{\boldsymbol{\beta}}_{\mathrm{O}}\right) \subseteq S^{*}$. Meanwhile, from Theorem 5.5 of Wang et al. (2014a) we have $\left\|\left(\widehat{\boldsymbol{\beta}}_{\lambda_{t}}\right)_{\overline{S^{*}}}\right\|_{0} \leq \widetilde{s}$. Hence, we have $\left\|\left(\widehat{\boldsymbol{\beta}}_{\lambda_{t}}-\widehat{\boldsymbol{\beta}}_{\mathrm{O}}\right)_{\overline{S^{*}}}\right\|_{0} \leq \widetilde{s}$, and Lemma 5.1 of Wang et al. (2014a) yields

$$
\begin{aligned}
& \widetilde{\mathcal{L}}_{\lambda_{t}}\left(\widehat{\boldsymbol{\beta}}_{\lambda_{t}}\right) \geq \widetilde{\mathcal{L}}_{\lambda_{t}}\left(\widehat{\boldsymbol{\beta}}_{\mathrm{O}}\right)+\nabla \widetilde{\mathcal{L}}_{\lambda_{t}}\left(\widehat{\boldsymbol{\beta}}_{\mathrm{O}}\right)^{T}\left(\widehat{\boldsymbol{\beta}}_{\lambda_{t}}-\widehat{\boldsymbol{\beta}}_{\mathrm{O}}\right) \\
&+\frac{\rho_{-}-\zeta_{-}}{2}\left\|\widehat{\boldsymbol{\beta}}_{\lambda_{t}}-\widehat{\boldsymbol{\beta}}_{\mathrm{O}}\right\|_{2}^{2}, \\
& \widetilde{\mathcal{L}}_{\lambda_{t}}\left(\widehat{\boldsymbol{\beta}}_{\mathrm{O}}\right) \geq \widetilde{\mathcal{L}}_{\lambda_{t}}\left(\widehat{\boldsymbol{\beta}}_{\lambda_{t}}\right)+\nabla \widetilde{\mathcal{L}}_{\lambda_{t}}\left(\widehat{\boldsymbol{\beta}}_{\lambda_{t}}\right)^{T}\left(\widehat{\boldsymbol{\beta}}_{\mathrm{O}}-\widehat{\boldsymbol{\beta}}_{\lambda_{t}}\right) \\
&+\frac{\rho_{-}-\zeta_{-}}{2}\left\|\widehat{\boldsymbol{\beta}}_{\mathrm{O}}-\widehat{\boldsymbol{\beta}}_{\lambda_{t}}\right\|_{2}^{2} .
\end{aligned}
$$

Meanwhile, by the convexity of $\ell_{1}$ norm, we have

$$
\begin{aligned}
& \lambda_{t}\left\|\widehat{\boldsymbol{\beta}}_{\lambda_{t}}\right\|_{1} \geq \lambda_{t}\left\|\widehat{\boldsymbol{\beta}}_{\mathrm{O}}\right\|_{1}+\lambda_{t}\left(\widehat{\boldsymbol{\beta}}_{\lambda_{t}}-\widehat{\boldsymbol{\beta}}_{\mathrm{O}}\right)^{T} \boldsymbol{\xi}_{\mathrm{O}}, \\
& \lambda_{t}\left\|\widehat{\boldsymbol{\beta}}_{\mathrm{O}}\right\|_{1} \geq \lambda_{t}\left\|\widehat{\boldsymbol{\beta}}_{\lambda_{t}}\right\|_{1}+\lambda_{t}\left(\widehat{\boldsymbol{\beta}}_{\mathrm{O}}-\widehat{\boldsymbol{\beta}}_{\lambda_{t}}\right)^{T} \widehat{\boldsymbol{\xi}}
\end{aligned}
$$

Adding (D.148)-(D.151), we obtain

$$
\begin{aligned}
0 \geq \underbrace{\left(\nabla \widetilde{\mathcal{L}}_{\lambda_{t}}\left(\widehat{\boldsymbol{\beta}}_{\lambda_{t}}\right)+\lambda_{t} \widehat{\boldsymbol{\xi}}\right)^{T}\left(\widehat{\boldsymbol{\beta}}_{\mathrm{O}}-\widehat{\boldsymbol{\beta}}_{\lambda_{t}}\right)}_{(\mathrm{i})} & +\underbrace{\left(\nabla \widetilde{\mathcal{L}}_{\lambda_{t}}\left(\widehat{\boldsymbol{\beta}}_{\mathrm{O}}\right)+\lambda_{t} \boldsymbol{\xi}_{\mathrm{O}}\right)^{T}\left(\widehat{\boldsymbol{\beta}}_{\lambda_{t}}-\widehat{\boldsymbol{\beta}}_{\mathrm{O}}\right)}_{(\mathrm{ii})} \\
& +\left(\rho_{-}-\zeta_{-}\right)\left\|\widehat{\boldsymbol{\beta}}_{\lambda_{t}}-\widehat{\boldsymbol{\beta}}_{\mathrm{O}}\right\|_{2}^{2} .
\end{aligned}
$$

According to (D.142), we have

$$
\begin{aligned}
\left(\widehat{\boldsymbol{\beta}}_{\lambda_{t}}-\widehat{\boldsymbol{\beta}}_{\mathrm{O}}\right)^{T}\left(\nabla \widetilde{\mathcal{L}}_{\lambda_{t}}\left(\widehat{\boldsymbol{\beta}}_{\lambda_{t}}\right)+\lambda_{t} \widehat{\boldsymbol{\xi}}\right) & \leq \max _{\boldsymbol{\beta}^{\prime} \in \Omega}\left\{\left(\widehat{\boldsymbol{\beta}}_{\lambda_{t}}-\boldsymbol{\beta}^{\prime}\right)^{T}\left(\nabla \widetilde{\mathcal{L}}_{\lambda_{t}}\left(\widehat{\boldsymbol{\beta}}_{\lambda_{t}}\right)+\lambda_{t} \widehat{\boldsymbol{\xi}}\right)\right\} \\
& \leq 0,
\end{aligned}
$$

which implies term (i) is nonnegative. Similarly, according to (D.143), term (ii) is also nonnegative. Hence we have

$$
\left(\rho_{-}-\zeta_{-}\right)\left\|\widehat{\boldsymbol{\beta}}_{\lambda_{t}}-\widehat{\boldsymbol{\beta}}_{\mathrm{O}}\right\|_{2}^{2} \leq 0 .
$$

By (4.3) of Wang et al. (2014a) we have $\rho_{-}-\boldsymbol{\zeta}_{-}>0$, which implies $\widehat{\boldsymbol{\beta}}_{\lambda_{t}}=\widehat{\boldsymbol{\beta}}_{\mathrm{O}}$. Thus, we conclude that $\widehat{\boldsymbol{\beta}}_{\lambda_{t}}$ is the oracle estimator $\widehat{\boldsymbol{\beta}}_{\mathrm{O}}$. Moreover, (D.145) implies that $\min _{j \in S^{*}}\left|\left(\widehat{\boldsymbol{\beta}}_{\mathrm{O}}\right)_{j}\right|>0$. Together with $\operatorname{supp}\left(\widehat{\boldsymbol{\beta}}_{\mathrm{O}}\right) \subseteq S^{*}$, we have $\operatorname{supp}\left(\widehat{\boldsymbol{\beta}}_{\lambda_{t}}\right)=\operatorname{supp}\left(\widehat{\boldsymbol{\beta}}_{\mathrm{O}}\right)=\operatorname{supp}\left(\boldsymbol{\beta}^{*}\right)$. 


\section{APPENDIX E: THEORETICAL RESULTS ABOUT SEMIPARAMETRIC ELLIPTICAL DESIGN REGRESSION}

In this section, we first introduce the Catoni's $M$-estimator of standard deviation, then we provide the detailed proofs of some necessary results on semiparametric elliptical design regression. ${ }^{2}$

E.1. Catoni's $\boldsymbol{M}$-Estimator of Standard Deviation. Catoni (2012) proposed a novel estimator for the mean and standard deviation of heavy-tail distributions. Let $\boldsymbol{Z}=\left(Z_{1}, \ldots, Z_{d+1}\right)$ be the elliptically distributed random vector defined in $\S 2.2$ of Wang et al. (2014a). We consider the estimator of the marginal mean $\mathbb{E}\left(Z_{j}\right)(j=1, \ldots, d+1)$. Let $h: \mathbb{R} \rightarrow \mathbb{R}$ be a continuous strictly increasing function satisfying

$$
-\log \left(1-x+x^{2} / 2\right) \leq h(x) \leq \log \left(1+x+x^{2} / 2\right) .
$$

For instance, we choose $h(\cdot)$ to be

$$
h(x)= \begin{cases}\log \left(1+x+x^{2} / 2\right), & \text { if } x \geq 0, \\ -\log \left(1-x+x^{2} / 2\right), & \text { otherwise. }\end{cases}
$$

Let $\delta \in(0,1)$ be such that $n \geq 2 \log (1 / \delta)$. We introduce

$$
a_{\delta}=\sqrt{2 \log (1 / \delta) /\left(n v+\frac{2 n v \log (1 / \delta)}{n-2 \log (1 / \delta)}\right)},
$$

where $v$ is an upper bound of $\operatorname{Var}\left(Z_{j}\right)$ for all $j$. Catoni's estimator of $\mathbb{E}\left(Z_{j}\right)$ is defined as $\widehat{\mu}_{j}=\widehat{\mu}_{j}(n, \delta)$ such that

$$
\sum_{i=1}^{n} h\left(\alpha_{\delta}\left(z_{i, j}-\widehat{\mu}_{j}\right)\right)=0, \quad j=1, \ldots, d+1,
$$

where $z_{i, j}$ is the $i$-th $(i=1, \ldots, n)$ realizations of $Z_{j}$. As $h(\cdot)$ is differentiable everywhere, we can solve (E.2) with Newton's method efficiently. Similarly we can estimate $\mathbb{E}\left(Z_{j}^{2}\right)$ with $\widehat{m}_{j}$ defined in a similar way. Then we obtain an estimator of the marginal standard deviation $\sigma_{j}$

$$
\widehat{\sigma}_{j}=\sqrt{\widehat{m}_{j}-\widehat{\mu}_{j}^{2}}, \quad j=1, \ldots, d+1 .
$$

E.2. Proof of Lemma C.3. To establish results concerning the smallest sparse eigenvalue for $\widehat{\mathbf{K}}_{\boldsymbol{X}}$, we need to prove several concentration results. The next lemma and proposition provide the concentration inequality for Catoni's estimator of marginal standard deviation, which is defined in (E.3).

\footnotetext{
${ }^{2} \S$ E.1, Lemma E.1 and Corollary E.2 come from an unpublished internal technical report. We provide them here for completeness.
} 
We first consider the estimator of variance in the following lemma.

Lemma E.1. Let $\boldsymbol{X}=\left(X_{1}, \ldots, X_{d}\right)^{T}$ be a random vector and $\mathbf{x}_{1}, \ldots, \mathbf{x}_{n}$ be $n$ independent realizations of $\boldsymbol{X}$ with $\operatorname{Var}\left(X_{j}\right)=v_{j}$ and $\mathbb{E}\left(X_{j}^{4}\right) \leq M$, for $j=1, \ldots, d$. We assume that

$$
\max _{1 \leq j \leq d}\left\{\left|\mathbb{E}\left(X_{j}\right)\right|\right\} \leq \mu_{\max }, \quad v_{\max }=\max _{1 \leq j \leq d}\left\{v_{j}\right\} .
$$

For the estimator $\widehat{v}_{j}=\widehat{m}_{j}-\widehat{\mu}_{j}^{2}$ with $\widehat{m}_{j}$ and $\widehat{\mu}_{j}$ defined in (E.2), if $n>5 \log d$, we have, with probability at least $1-2 d^{-3}$,

$$
\max _{1 \leq j \leq d}\left\{\left|v_{j}-\widehat{v}_{j}\right|\right\} \leq C \sqrt{\frac{\log d}{n}}
$$

where $C$ is a constant.

Proof. For $j \in\{1, \ldots, d\}$, we use $\widehat{m}_{j}$ to estimate $\mathbb{E}\left(X_{j}^{2}\right)$. Catoni (2012) showed that

$$
\mathbb{P}\left(\left|\widehat{m}_{j}-\mathbb{E}\left(X_{j}^{2}\right)\right|>t\right) \leq \exp \left(-\frac{n t^{2}}{M}\right) .
$$

Taking a union bound, we have

$$
\mathbb{P}\left(\max _{1 \leq j \leq d}\left\{\left|\widehat{m}_{j}-\mathbb{E}\left(X_{j}^{2}\right)\right|\right\}>t\right) \leq d \exp \left(-\frac{n t^{2}}{M}\right),
$$

or equivalently, with probability at least $1-d^{-3}$,

$$
\max _{1 \leq j \leq d}\left\{\left|\widehat{m}_{j}-\mathbb{E}\left(X_{j}^{2}\right)\right|\right\} \leq 2 \sqrt{M} \sqrt{\frac{\log d}{n}} .
$$

Meanwhile, we use $\widehat{\mu}_{j}$ to estimate $\mathbb{E}\left(X_{j}\right)$. By similar arguments as above, we have

$$
\max _{1 \leq j \leq d}\left\{\left|\widehat{\mu}_{j}-\mathbb{E}\left(X_{j}\right)\right|\right\} \leq 2 \sqrt{v_{\max }} \sqrt{\frac{\log d}{n}}
$$

with probability at least $1-d^{-3}$.

Note that

$$
\max _{1 \leq j \leq d}\left\{\left|\widehat{\mu}_{j}^{2}-\left(\mathbb{E}\left(X_{j}\right)\right)^{2}\right|\right\} \leq \max _{1 \leq j \leq d}\left\{\left|\widehat{\mu}_{j}-\mathbb{E}\left(X_{j}\right)\right|\right\} \cdot \max _{1 \leq j \leq d}\left\{\left|\widehat{\mu}_{j}+\mathbb{E}\left(X_{j}\right)\right|\right\} .
$$

Since we assume that $\max _{1 \leq j \leq d}\left\{\mathbb{E}\left(X_{j}\right)\right\} \leq \mu_{\max }$, we have

$$
\max _{1 \leq j \leq d}\left\{\left|\widehat{\mu}_{j}^{2}-\left(\mathbb{E}\left(X_{j}\right)\right)^{2}\right|\right\} \leq\left(4 \mu_{\max }+4 \sqrt{v_{\max }} \sqrt{\frac{\log d}{n}}\right) \cdot \sqrt{v_{\max }} \sqrt{\frac{\log d}{n}}
$$


with probability at least $1-d^{-3}$. Since $\log d / n<1$, from (E.6) we have,

$$
\max _{1 \leq j \leq d}\left\{\left|\widehat{\mu}_{j}^{2}-\left(\mathbb{E}\left(X_{j}\right)\right)^{2}\right|\right\} \leq\left(4 \mu_{\max }+4 \sqrt{v_{\max }}\right) \cdot \sqrt{v_{\max }} \sqrt{\frac{\log d}{n}} .
$$

Combining (E.4) and (E.7), we have, with probability at least $1-2 d^{-3}$,

$$
\max _{1 \leq j \leq d}\left\{\left|\widehat{m}_{j}-\widehat{\mu}_{j}^{2}-\operatorname{Var}\left(X_{j}\right)\right|\right\} \leq C \sqrt{\frac{\log d}{n}},
$$

where $C=2 \sqrt{M}+\left(4 \mu_{\max }+4 \sqrt{v_{\max }}\right) \sqrt{v_{\max }}$.

We use $\widehat{\sigma}_{j}=\sqrt{\widehat{v}_{j}}$ to estimate $\sigma_{j}=\sqrt{v_{j}}$. Using Lemma E.1, we derive a concentration inequality for $\widehat{\sigma}_{j}$ in the following corollary.

Corollary E.2. Let $\sigma_{j}=\sqrt{v_{j}}$ and $\widehat{\sigma}_{j}=\sqrt{\widehat{v}_{j}}$ for $j=1, \ldots, d$. By assuming $\sigma_{j} \geq \sigma_{\min }>0$ for all $j=1, \ldots, d$, we have, with probability at least $1-2 d^{-3}$,

$$
\max _{1 \leq j \leq d}\left\{\left|\sigma_{j}-\widehat{\sigma}_{j}\right|\right\} \leq C \sqrt{\frac{\log d}{n}}
$$

where $C$ is a constant.

Proof. By Lemma E.1, we have, with probability at least $1-2 d^{-3}$,

$$
\max _{1 \leq j \leq d}\left\{\left|v_{j}-\widehat{v}_{j}\right|\right\} \leq C \sqrt{\frac{\log d}{n}}
$$

Since $\left|v_{j}-\widehat{v}_{j}\right|=\left|\sigma_{j}-\widehat{\sigma}_{j}\right| \cdot\left|\sigma_{j}+\widehat{\sigma}_{j}\right|$, it follows that

$$
\max _{1 \leq j \leq d}\left\{\left|\sigma_{j}-\widehat{\sigma}_{j}\right|\right\} \leq \frac{C}{\min _{1 \leq j \leq d}\left\{\left|\sigma_{j}+\widehat{\sigma}_{j}\right|\right\}} \sqrt{\frac{\log d}{n}} \leq \frac{C}{\sigma_{\min }} \sqrt{\frac{\log d}{n}} .
$$

As we assume that $\sigma_{j}>\sigma_{\min }$ for all $j$, we conclude the proof.

Before we establish the sparse eigenvalue condition for $\widehat{\mathbf{K}}_{\boldsymbol{X}}$, we provide a concentration result of $\widehat{\mathbf{R}}_{\boldsymbol{X}}$ in the following lemma.

Lemma E.3 (Han and Liu (2013)). Let $\mathbf{x}_{1}, \ldots, \mathbf{x}_{n}$ be $n$ realizations of a random vector $\boldsymbol{X} \sim \mathrm{EC}_{d}\left(0, \boldsymbol{\Sigma}_{\boldsymbol{X}}, \Xi\right)$ as in Definition A.1. We assume that the smallest eigenvalue of the generalized correlation matrix $\boldsymbol{\Sigma}_{\boldsymbol{X}}^{0}$ is strictly positive. Under the sign sub-Gaussian condition (see Han and Liu (2013) for more details), the correlation matrix estimator $\widehat{\mathbf{R}}_{\boldsymbol{X}}$ defined in (A.1) satisfies that, with probability at least $1-2 d^{-1}-d^{-2}$,

$$
\sup _{\|\boldsymbol{v}\|_{0} \leq s}\left\{\frac{\left|\boldsymbol{v}^{T}\left(\widehat{\mathbf{R}}_{\boldsymbol{X}}-\boldsymbol{\Sigma}_{\boldsymbol{X}}^{0}\right) \boldsymbol{v}\right|}{\|\boldsymbol{v}\|_{2}^{2}}\right\} \leq C \sqrt{\frac{s \log d}{n}}
$$


for $s \in\{1, \ldots, d\}$ and a sufficiently large $n$.

We now prove Lemma C.3.

Proof. Here we denote the diagonal matrix that has $x_{1}, \ldots, x_{d}$ on its diagonal by $\operatorname{diag}\left(x_{1}, \ldots, x_{d}\right)$. Let $\mathbf{D}=\operatorname{diag}\left(\sigma_{1}, \ldots, \sigma_{d}\right)$ and $\widehat{\mathbf{D}}=\operatorname{diag}\left(\widehat{\sigma}_{1}, \ldots, \widehat{\sigma}_{d}\right)$. First we consider the smallest sparse eigenvalue, which satisfies

$$
\begin{aligned}
\rho_{-}\left(\nabla^{2} \mathcal{L}, s\right) & =\inf _{\|\boldsymbol{v}\|_{0} \leq s}\left\{\frac{\boldsymbol{v}^{T} \widehat{\mathbf{K}}_{\boldsymbol{X}} \boldsymbol{v}}{\|\boldsymbol{v}\|_{2}^{2}}\right\} \\
& =\inf _{\|\boldsymbol{v}\|_{0} \leq s}\left\{\frac{(\widehat{\mathbf{D}} \boldsymbol{v})^{T} \widehat{\mathbf{R}}_{\boldsymbol{X}}(\widehat{\mathbf{D}} \boldsymbol{v})}{\|\widehat{\mathbf{D}} \boldsymbol{v}\|_{2}^{2}} \cdot \frac{\|\widehat{\mathbf{D}} \boldsymbol{v}\|_{2}^{2}}{\|\boldsymbol{v}\|_{2}^{2}}\right\} \\
& \geq \inf _{\|\boldsymbol{v}\|_{0} \leq s}\left\{\frac{\boldsymbol{v}^{T} \widehat{\mathbf{R}}_{\boldsymbol{X}} \boldsymbol{v}}{\|\boldsymbol{v}\|_{2}^{2}}\right\} \cdot \min _{1 \leq j \leq d}\left\{\widehat{\sigma}_{j}\right\} .
\end{aligned}
$$

The first term on the right-hand side of (E.8) is the smallest sparse eigenvalue of $\widehat{\mathbf{R}}_{\boldsymbol{X}}$. Since we have from Lemma E.3 that, with probability at least $1-2 d^{-1}-d^{-2}$

$$
\sup _{\|\boldsymbol{v}\|_{0} \leq s}\left\{\frac{\left|\boldsymbol{v}^{T}\left(\widehat{\mathbf{R}}_{\boldsymbol{X}}-\boldsymbol{\Sigma}_{\boldsymbol{X}}^{0}\right) \boldsymbol{v}\right|}{\|\boldsymbol{v}\|_{2}^{2}}\right\} \leq C \sqrt{\frac{s \log d}{n}} .
$$

Then for a sufficiently large $n$, we have

$$
\boldsymbol{v}^{T}\left(\boldsymbol{\Sigma}_{\boldsymbol{X}}^{0}-\widehat{\mathbf{R}}_{\boldsymbol{X}}\right) \boldsymbol{v} \leq C \sqrt{\frac{s \log d}{n}} \leq \frac{1}{2} \Lambda_{\min }\left(\boldsymbol{\Sigma}_{\boldsymbol{X}}^{0}\right), \quad \text { for } \quad\|\boldsymbol{v}\|_{0} \leq s .
$$

Here $\Lambda_{\text {min }}\left(\boldsymbol{\Sigma}_{\boldsymbol{X}}^{0}\right)$ denotes the smallest eigenvalue of $\boldsymbol{\Sigma}_{\boldsymbol{X}}^{0}$, which is strictly positive by assumption. Then we obtain

$$
\frac{1}{2} \Lambda_{\min }\left(\boldsymbol{\Sigma}_{\boldsymbol{X}}^{0}\right) \leq \boldsymbol{v}^{T} \boldsymbol{\Sigma}_{\boldsymbol{X}}^{0} \boldsymbol{v}-\frac{1}{2} \Lambda_{\min }\left(\boldsymbol{\Sigma}_{\boldsymbol{X}}^{0}\right) \leq \boldsymbol{v}^{T} \widehat{\mathbf{R}}_{\boldsymbol{X}} \boldsymbol{v}, \quad \text { for } \quad\|\boldsymbol{v}\|_{0} \leq s .
$$

Taking infimum over both sides, we get

$$
\inf _{\|\boldsymbol{v}\|_{0} \leq s}\left\{\frac{\boldsymbol{v}^{T} \widehat{\mathbf{R}}_{\boldsymbol{X}} \boldsymbol{v}}{\|\boldsymbol{v}\|_{2}^{2}}\right\} \geq \frac{1}{2} \Lambda_{\min }\left(\boldsymbol{\Sigma}_{\boldsymbol{X}}^{0}\right)>0 .
$$

We now consider $\min _{1 \leq j \leq d}\left\{\widehat{\sigma}_{j}\right\}$ in (E.8). In Corollary E.2 we prove that, with probability at least $1-2 d^{-3}$,

$$
\left|\sigma_{j}-\widehat{\sigma}_{j}\right| \leq C^{\prime} \sqrt{\frac{\log d}{n}}, \quad \text { for } 1 \leq j \leq d,
$$


where $C^{\prime}$ is a constant. For a sufficiently large $n$, we have

$$
\widehat{\sigma}_{j} \geq \frac{1}{2} \sigma_{j}>0, \quad \text { for } 1 \leq j \leq d
$$

with the same probability. Taking minimum over both sides, we get

$$
\min _{1 \leq j \leq d}\left\{\widehat{\sigma}_{j}\right\} \geq \frac{1}{2} \min _{1 \leq j \leq d}\left\{\sigma_{j}\right\}>0
$$

with probability at least $1-2 d^{-2}$. Plugging (E.9) and (E.10) into the righthand side of $\left(\right.$ E.8), we reach the conclusion that $\rho_{-}\left(\nabla^{2} \mathcal{L}, s\right)>0$ holds with probability at least $1-2 d^{-1}-3 d^{-2}$.

Now we consider the largest sparse eigenvalue, which satisfies

$$
\begin{aligned}
\rho_{+}\left(\nabla^{2} \mathcal{L}, s\right) & =\sup _{\|\boldsymbol{v}\|_{0} \leq s}\left\{\frac{\boldsymbol{v}^{T} \widehat{\mathbf{K}}_{\boldsymbol{X}} \boldsymbol{v}}{\|\boldsymbol{v}\|_{2}^{2}}\right\} \\
& =\sup _{\|\boldsymbol{v}\|_{0} \leq s}\left\{\frac{(\widehat{\mathbf{D}} \boldsymbol{v})^{T} \widehat{\mathbf{R}}_{\boldsymbol{X}}(\widehat{\mathbf{D}} \boldsymbol{v})}{\|\widehat{\mathbf{D}} \boldsymbol{v}\|_{2}^{2}} \cdot \frac{\|\widehat{\mathbf{D}} \boldsymbol{v}\|_{2}^{2}}{\|\boldsymbol{v}\|_{2}^{2}}\right\} \\
& \leq \sup _{\|\boldsymbol{v}\|_{0} \leq s}\left\{\frac{\boldsymbol{v}^{T} \widehat{\mathbf{R}}_{\boldsymbol{X}} \boldsymbol{v}}{\|\boldsymbol{v}\|_{2}^{2}}\right\} \cdot \max _{1 \leq j \leq d}\left\{\widehat{\sigma}_{j}\right\} .
\end{aligned}
$$

The first term on the right-hand side of (E.11) is the largest sparse eigenvalue of $\widehat{\mathbf{R}}_{\boldsymbol{X}}$. Since we have from Lemma E.3 that, with probability at least $1-2 d^{-1}-d^{-2}$,

$$
\sup _{\|\boldsymbol{v}\|_{0} \leq s}\left\{\frac{\left|\boldsymbol{v}^{T}\left(\widehat{\mathbf{R}}_{\boldsymbol{X}}-\boldsymbol{\Sigma}_{\boldsymbol{X}}^{0}\right) \boldsymbol{v}\right|}{\|\boldsymbol{v}\|_{2}^{2}}\right\} \leq C \sqrt{\frac{s \log d}{n}} .
$$

Then for a sufficiently large $n$, we have

$$
\boldsymbol{v}^{T}\left(\widehat{\mathbf{R}}_{\boldsymbol{X}}-\boldsymbol{\Sigma}_{\boldsymbol{X}}^{0}\right) \boldsymbol{v} \leq C \sqrt{\frac{s \log d}{n}} \leq \frac{1}{2} \Lambda_{\max }\left(\boldsymbol{\Sigma}_{\boldsymbol{X}}^{0}\right), \quad \text { for }\|\boldsymbol{v}\|_{0} \leq s .
$$

Here $\Lambda_{\max }\left(\boldsymbol{\Sigma}_{\boldsymbol{X}}^{0}\right)$ denotes the largest eigenvalue of $\boldsymbol{\Sigma}_{\boldsymbol{X}}^{0}$. Then we obtain

$$
\boldsymbol{v}^{T} \widehat{\mathbf{R}}_{\boldsymbol{X}} \boldsymbol{v} \leq \boldsymbol{v}^{T} \boldsymbol{\Sigma}_{\boldsymbol{X}}^{0} \boldsymbol{v}+\frac{1}{2} \Lambda_{\max }\left(\boldsymbol{\Sigma}_{\boldsymbol{X}}^{0}\right) \leq \frac{3}{2} \Lambda_{\max }\left(\boldsymbol{\Sigma}_{\boldsymbol{X}}^{0}\right), \quad \text { for }\|\boldsymbol{v}\|_{0} \leq s .
$$

Taking supremum over both sides, we get

$$
\sup _{\|\boldsymbol{v}\|_{0} \leq s}\left\{\frac{\boldsymbol{v}^{T} \widehat{\mathbf{R}}_{\boldsymbol{X}} \boldsymbol{v}}{\|\boldsymbol{v}\|_{2}^{2}}\right\} \leq \frac{1}{2} \Lambda_{\max }\left(\boldsymbol{\Sigma}_{\boldsymbol{X}}^{0}\right)<+\infty .
$$

We now consider $\max _{1 \leq j \leq d}\left\{\widehat{\sigma}_{j}\right\}$ in (E.11). In Corollary E.2 we prove that, 
with probability at least $1-2 d^{-3}$,

$$
\left|\sigma_{j}-\widehat{\sigma}_{j}\right| \leq C^{\prime} \sqrt{\frac{\log d}{n}}, \quad \text { for } 1 \leq j \leq d,
$$

where $C^{\prime}$ is a constant. For a sufficiently large $n$, we have

$$
\widehat{\sigma}_{j} \leq \frac{3}{2} \sigma_{j}<+\infty, \quad \text { for } 1 \leq j \leq d
$$

with the same probability. Taking minimum over both sides, we get

$$
\max _{1 \leq j \leq d}\left\{\widehat{\sigma}_{j}\right\} \leq \frac{3}{2} \max _{1 \leq j \leq d}\left\{\sigma_{j}\right\}<+\infty
$$

with probability at least $1-2 d^{-2}$. Plugging (E.12) and (E.13) into the righthand side of (E.11), we reach the conclusion that $\rho_{+}\left(\nabla^{2} \mathcal{L}, s\right)<+\infty$ holds with probability at least $1-2 d^{-1}-3 d^{-2}$. Thus we conclude the proof.

\section{E.3. Proof of Lemma C.2.}

Proof. For semiparametric elliptical design regression, we have

$$
\nabla \mathcal{L}\left(\boldsymbol{\beta}^{*}\right)=\widehat{\mathbf{K}}_{\boldsymbol{X}, Y}-\widehat{\mathbf{K}}_{\boldsymbol{X}} \boldsymbol{\beta}^{*}=\widehat{\mathbf{K}}_{\boldsymbol{X}, Y}-\boldsymbol{\Sigma}_{\boldsymbol{X}, Y}+\boldsymbol{\Sigma}_{\boldsymbol{X}, Y}-\widehat{\mathbf{K}}_{\boldsymbol{X}} \boldsymbol{\beta}^{*},
$$

where $\widehat{\mathbf{K}}_{\boldsymbol{X}} \in \mathbb{R}^{d \times d}$ and $\widehat{\mathbf{K}}_{\boldsymbol{X}, Y} \in \mathbb{R}^{d \times 1}$ are the submatrices of $\widehat{\mathbf{K}}_{\boldsymbol{Z}} \in \mathbb{R}^{(d+1) \times(d+1)}$ defined in (B.1). Since $\mathbb{E}(Y \mid \boldsymbol{X}=\mathbf{x})=\mathbf{x}^{T} \boldsymbol{\beta}^{*}$, we have

$$
\boldsymbol{\Sigma}_{\boldsymbol{X}, Y}=\mathbb{E}(\boldsymbol{X} Y)=\mathbb{E}\left(\boldsymbol{X} \boldsymbol{X}^{T} \boldsymbol{\beta}^{*}\right)=\boldsymbol{\Sigma}_{\boldsymbol{X}} \boldsymbol{\beta}^{*} .
$$

Hence we have

$$
\begin{aligned}
\left\|\nabla \mathcal{L}\left(\boldsymbol{\beta}^{*}\right)\right\|_{\infty} & =\left\|\widehat{\mathbf{K}}_{\boldsymbol{X}, Y}-\boldsymbol{\Sigma}_{\boldsymbol{X}, Y}+\boldsymbol{\Sigma}_{\boldsymbol{X}} \boldsymbol{\beta}^{*}-\widehat{\mathbf{K}}_{\boldsymbol{X}} \boldsymbol{\beta}^{*}\right\|_{\infty} \\
& \leq\left\|\widehat{\mathbf{K}}_{\boldsymbol{X}, Y}-\boldsymbol{\Sigma}_{\boldsymbol{X}, Y}\right\|_{\infty}+\left\|\boldsymbol{\Sigma}_{\boldsymbol{X}} \boldsymbol{\beta}^{*}-\widehat{\mathbf{K}}_{\boldsymbol{X}} \boldsymbol{\beta}^{*}\right\|_{\infty} .
\end{aligned}
$$

Before we upper bound the two terms on the right-hand side, we establish a concentration inequality for $\widehat{\mathbf{K}}_{\boldsymbol{Z}}$. Let $\mathbf{D}_{\boldsymbol{Z}}=\operatorname{diag}\left(\sigma_{1}, \ldots, \sigma_{d+1}\right)$ and $\widehat{\mathbf{D}}_{\boldsymbol{Z}}=$ $\operatorname{diag}\left(\widehat{\sigma}_{1}, \ldots, \widehat{\sigma}_{d+1}\right)$, where $\sigma_{1}, \ldots, \sigma_{d+1}$ are the marginal standard deviations of $\boldsymbol{Z} \in \mathbb{R}^{(d+1)}=(Y, \boldsymbol{X})^{T}$ while $\widehat{\sigma}_{1}, \ldots, \widehat{\sigma}_{d+1}$ are the corresponding Catoni's estimators defined in (E.3). We have

$$
\boldsymbol{\Sigma}_{Z}=\mathbf{D}_{Z} \boldsymbol{\Sigma}_{Z}^{0} \mathbf{D}_{Z}, \quad \widehat{\mathbf{K}}_{Z}=\widehat{\mathbf{D}}_{Z} \widehat{\mathbf{R}}_{Z} \widehat{\mathbf{D}}_{Z}
$$

where $\widehat{\mathbf{R}}_{\boldsymbol{Z}}$ is the rank-based estimator of the generalized correlation matrix $\boldsymbol{\Sigma}_{\boldsymbol{Z}}^{0}$ defined in (A.1). Han and Liu (2012) proved that, with probability at least at least $1-(d+1)^{-5 / 2}$,

$$
\left\|\widehat{\mathbf{R}}_{\boldsymbol{Z}}-\boldsymbol{\Sigma}_{\boldsymbol{Z}}^{0}\right\|_{\max } \leq C \sqrt{\frac{\log (d+1)}{n}},
$$


where $\|\mathbf{M}\|_{\max }=\max _{1 \leq i, j \leq d}\left\{\left|M_{i, j}\right|\right\}$ for $\mathbf{M} \in \mathbb{R}^{d \times d}$. We have

$$
\begin{aligned}
&\left\|\widehat{\mathbf{D}}_{\boldsymbol{Z}} \widehat{\mathbf{R}}_{\boldsymbol{Z}} \widehat{\mathbf{D}}_{\boldsymbol{Z}}-\mathbf{D}_{\boldsymbol{Z}} \boldsymbol{\Sigma}_{\boldsymbol{Z}}^{0} \mathbf{D}_{\boldsymbol{Z}}\right\|_{\max } \\
&=\left\|\mathbf{D}_{\boldsymbol{Z}}\left(\widehat{\mathbf{R}}_{\boldsymbol{Z}}-\boldsymbol{\Sigma}_{\boldsymbol{Z}}^{0}\right) \mathbf{D}_{\boldsymbol{Z}}+\left(\widehat{\mathbf{D}}_{\boldsymbol{Z}}-\mathbf{D}_{\boldsymbol{Z}}\right) \widehat{\mathbf{R}}_{\boldsymbol{Z}} \mathbf{D}_{\boldsymbol{Z}}+\widehat{\mathbf{D}}_{\boldsymbol{Z}} \widehat{\mathbf{R}}_{\boldsymbol{Z}}\left(\widehat{\mathbf{D}}_{\boldsymbol{Z}}-\mathbf{D}_{\boldsymbol{Z}}\right)\right\|_{\max } \\
& \leq\left\|\mathbf{D}_{\boldsymbol{Z}}\left(\widehat{\mathbf{R}}_{\boldsymbol{Z}}-\boldsymbol{\Sigma}_{\boldsymbol{Z}}^{0}\right) \mathbf{D}_{\boldsymbol{Z}}\right\|_{\max }+\left\|\left(\widehat{\mathbf{D}}_{\boldsymbol{Z}}-\mathbf{D}_{\boldsymbol{Z}}\right) \widehat{\mathbf{R}}_{\boldsymbol{Z}} \mathbf{D}_{\boldsymbol{Z}}\right\|_{\max } \\
&+\left\|\widehat{\mathbf{D}}_{\boldsymbol{Z}} \widehat{\mathbf{R}}_{\boldsymbol{Z}}\left(\widehat{\mathbf{D}}_{\boldsymbol{Z}}-\mathbf{D}_{\boldsymbol{Z}}\right)\right\|_{\max } \\
& \leq\left\|\mathbf{D}_{\boldsymbol{Z}}\right\|_{\max }^{2}\left\|\widehat{\mathbf{R}}_{\boldsymbol{Z}}-\boldsymbol{\Sigma}_{\boldsymbol{Z}}^{0}\right\|_{\max }^{2}+\left\|\mathbf{D}_{\boldsymbol{Z}}\right\|_{\max }\left\|\widehat{\mathbf{D}}_{\boldsymbol{Z}}-\mathbf{D}_{\boldsymbol{Z}}\right\|_{\max } \\
&+\left\|\widehat{\mathbf{D}}_{\boldsymbol{Z}}\right\|_{\max }\left\|\widehat{\mathbf{D}}_{\boldsymbol{Z}}-\mathbf{D}_{\boldsymbol{Z}}\right\|_{\max } .
\end{aligned}
$$

Following similar arguments in Corollary E.2, we have

$$
\left\|\widehat{\mathbf{D}}_{\boldsymbol{Z}}-\mathbf{D}_{\boldsymbol{Z}}\right\|_{\max } \leq C \sqrt{\frac{\log (d+1)}{n}}, \quad\left\|\widehat{\mathbf{D}}_{\boldsymbol{Z}}\right\|_{\max } \leq\left\|\mathbf{D}_{\boldsymbol{Z}}\right\|_{\max }+C \sqrt{\frac{\log (d+1)}{n}}
$$

with probability at least $1-2(d+1)^{-3}$. We assume that $\sigma_{j}(1 \leq j \leq$ $d+1$ ) is upper bounded, from (E.14) we have, with probability at least $1-(d+1)^{-5 / 2}-2(d+1)^{-3}$,

$$
\left\|\boldsymbol{\Sigma}_{\boldsymbol{Z}}-\widehat{\mathbf{K}}_{\boldsymbol{Z}}\right\|_{\max } \leq C \sqrt{\frac{\log (d+1)}{n}},
$$

which implies that with the same probability,

$$
\begin{aligned}
& \left\|\widehat{\mathbf{K}}_{\boldsymbol{X}, Y}-\boldsymbol{\Sigma}_{\boldsymbol{X}, Y}\right\|_{\infty} \leq C \sqrt{\frac{\log (d+1)}{n}}, \\
& \left\|\boldsymbol{\Sigma}_{\boldsymbol{X}} \boldsymbol{\beta}^{*}-\widehat{\mathbf{K}}_{\boldsymbol{X}} \boldsymbol{\beta}^{*}\right\|_{\infty} \leq\left\|\boldsymbol{\beta}^{*}\right\|_{1}\left\|\boldsymbol{\Sigma}_{\boldsymbol{X}}-\widehat{\mathbf{K}}_{\boldsymbol{X}}\right\|_{\max } \leq C\left\|\boldsymbol{\beta}^{*}\right\|_{1} \sqrt{\frac{\log (d+1)}{n}} .
\end{aligned}
$$

Then we reach the conclusion.

\section{APPENDIX F: DETAILED SETTINGS OF NUMERICAL EXPERIMENTS}

The detailed settings of the first numerical experiment in $\S 7$ of Wang et al. (2014a) are as follows:

- The design matrix $\mathbf{X} \in \mathbb{R}^{n \times d}$ contains $n=500$ independent realizations of a random vector $\boldsymbol{X} \in \mathbb{R}^{d}$ with $d=2500$, which follows a $t$-distribution with 5 degrees of freedom, zero mean and correlation matrix $\boldsymbol{\Sigma}_{\boldsymbol{X}}^{0}$. We set the correlation matrix $\boldsymbol{\Sigma}_{\boldsymbol{X}}^{0}$ to be $\left(\boldsymbol{\Sigma}_{\boldsymbol{X}}^{0}\right)_{i, j}=0.8^{|i-j|}(1 \leq i, j \leq d)$. Meanwhile, in the $i$-th data sample the response $y_{i}$ follows a univariate $t$-distribution with 5 degrees of freedom, mean $\mathbf{x}_{i}^{T} \boldsymbol{\beta}^{*}$ and variance 0.01 . Here $\mathbf{x}_{i}^{T}$ is the $i$-th row of the design matrix $\mathbf{X}$, and $\boldsymbol{\beta}^{*}$ is the true parameter vector specified as follows. 
- For the true parameter vector $\boldsymbol{\beta}^{*} \in \mathbb{R}^{d}$, we set its first 100 coordinates to be independent realizations of a standard univariate Gaussian distribution (zero mean and unit variance), and other coordinates to be zero, i.e., we set $s^{*}=\left|\operatorname{supp}\left(\boldsymbol{\beta}^{*}\right)\right|=100$.

- For the sequence of regularization parameters $\left\{\lambda_{t}\right\}_{t=0}^{N}$, we set $\lambda_{\text {tgt }}=$ 0.05 by cross-validation. Remind $\lambda_{0}=\|\nabla \mathcal{L}(\mathbf{0})\|_{\infty}=\left\|\widehat{\mathbf{K}}_{\boldsymbol{X}, Y}\right\|_{\infty}$, where $\widehat{\mathbf{K}}_{\boldsymbol{X}, Y} \in \mathbb{R}^{d}$ is in (B.1). We fix the random seed in MATLAB to be 2 . In this setting, we observe $\lambda_{0}=2.8516$. We set $\eta=0.9015$, so that the total number of regularization parameters is $N=\log \left(\lambda_{\text {tgt }} / \lambda_{0}\right) / \log \eta=39$.

- For the MCP penalty defined in (2.2) of Wang et al. (2014a), we set $b=1.1$. Meanwhile, we set the optimization precision within the $N$-th path following stage to be $\epsilon_{\mathrm{opt}}=10^{-6}$, and $L_{\mathrm{min}}=10^{-6}$.

The detailed settings of the second numerical experiment in $\S 7$ are as follows:

- The design matrix $\mathbf{X} \in \mathbb{R}^{n \times d}$ contains $n=200$ independent realizations of a random vector $\boldsymbol{X} \in \mathbb{R}^{d}$ with $d=2000$, which follows a zero mean Gaussian distribution with covariance matrix $\left(\boldsymbol{\Sigma}_{\boldsymbol{X}}\right)_{i, j}=0.9 \cdot \mathbb{1}(i \neq j)+$ $\mathbb{1}(i=j)$. Meanwhile, we set $\mathbf{y}-\mathbf{X} \boldsymbol{\beta}^{*}$ to be an $n$-dimensional Gaussian random vector with zero mean and covariance matrix $\mathbf{I} ; \boldsymbol{\beta}^{*}$ is set to be zero on its first 1990 dimensions, and takes +2 and -2 with equal probability on its last 10 dimensions.

- For the sequence of regularization parameters $\left\{\lambda_{t}\right\}_{t=0}^{N}$, we set $\lambda_{\text {tgt }}$ by cross-validation, and $\lambda_{0}=\|\nabla \mathcal{L}(\mathbf{0})\|_{\infty}=\left\|\mathbf{X}^{T} \mathbf{y}\right\|_{\infty} / n$. Other parameters are set to be the same as in the previous experiment.

- We compare with LLA (Zou and Li, 2008), the calibrated CCCP (Wang et al., 2013), SparseNet (Mazumder et al., 2011), and the multi-stage convex relaxation method (Zhang, 2010b; Zhang et al., 2013). For SparseNet we use the same sequence of regularization parameters as in our setting. For the other procedures, we employ the glmnet package (Friedman et al., 2010) to compute the Lasso problem in (6.1) of Wang et al. (2014a) at each stage.

- For the multi-stage convex relaxation method, we set the maximum number of stages to be 20. For the calibrated CCCP, we set the tuning parameter $\tau=1 / \log (n)$ as suggested by Wang et al. (2013). To be fair, each method selects its most suitable regularization parameter using cross-validation. We repeat the experiment for 1000 times. 


\section{REFERENCES}

Bickel, P. J., Ritov, Y. and Tsybakov, A. B. (2009). Simultaneous analysis of lasso and Dantzig selector. Annals of Statistics 37 1705-1732.

CAndés, E. and TAO, T. (2007). The Dantzig selector: Statistical estimation when $p$ is much larger than $n$. Annals of Statistics 2313-2351.

Catoni, O. (2012). Challenging the empirical mean and empirical variance: a deviation study. Annales de l'Institut Henri Poincaré, Probabilités et Statistiques 48 1148-1185.

Friedman, J., Hastie, T. and Tibshirani, R. (2010). Regularization paths for generalized linear models via coordinate descent. Journal of Statistical Software 331.

HAN, F. and LiU, H. (2012). Transelliptical component analysis. In Advances in Neural Information Processing Systems 25.

HAN, F. and LiU, H. (2013). Optimal rates of convergence of transelliptical component analysis. arXiv preprint arXiv:1305.6916.

KoltchinskiI, V. (2009a). The Dantzig selector and sparsity oracle inequalities. Bernoulli 15 799-828.

Liu, H., Han, F. and Zhang, C.-H. (2012). Transelliptical graphical models. In Advances in Neural Information Processing Systems 25.

Loh, P.-L. and Wainwright, M. J. (2013). Regularized $M$-estimators with nonconvexity: Statistical and algorithmic theory for local optima. arXiv preprint arXiv:1305.2436.

Mazumder, R., Friedman, J. H. and Hastie, T. (2011). Sparsenet: Coordinate descent with nonconvex penalties. Journal of the American Statistical Association 106.

Negahban, S. N., Ravikumar, P., Wainwright, M. J. and Yu, B. (2012). A unified framework for high-dimensional analysis of $m$-estimators with decomposable regularizers. Statistical Science $\mathbf{2 7} 538-557$.

Nesterov, Y. (2004). Introductory lectures on convex optimization: A basic course, vol. 87. Springer.

Nesterov, Y. (2013). Gradient methods for minimizing composite functions. Mathematical Programming 140 125-161.

WAINWRIGHT, M. (2009). Sharp thresholds for high dimensional and noisy sparsity recovery using $\ell_{1}$-constrained quadratic programming. IEEE Transactions on Information Theory $552183-2201$.

WANG, L., KIM, Y. and Li, R. (2013). Calibrating nonconvex penalized regression in ultra-high dimension. Annals of Statistics 41 2505-2536.

WANG, Z., LiU, H. and ZHANG, T. (2014a). Optimal computational and statistical rates of convergence for sparse nonconvex learning problems. arXiv preprint arXiv:1306.4960.

ZhANG, T. (2010b). Analysis of multi-stage convex relaxation for sparse regularization. Journal of Machine Learning Research 11 1087-1107.

ZhANG, C.-H. and HuAng, J. (2008). The sparsity and bias of the lasso selection in high-dimensional linear regression. Annals of Statistics 36 1567-1594.

Zhang, T. (2009). Some sharp performance bounds for least squares regression with $\ell_{1}$ regularization. Annals of Statistics 37 2109-2144.

ZhAng, T. (2013). Multi-stage convex relaxation for feature selection. Bernoulli 19 2277-2293.

Zou, H. and LI, R. (2008). One-step sparse estimates in nonconcave penalized likelihood models. Annals of Statistics 361509. 
Department of Operations Research and Financial Engineering

Princeton University

Princeton, New Jersey 08544

USA

E-MAIL: zhaoran@princeton.edu hanliu@princeton.edu
Department of Statistics Rutgers University

Piscataway, New Jersey 08854 USA

E-MAIL: tzhang@stat.rutgers.edu 